DIVISION OF THE HUMANITIES AND SOCIAL SCIENCES

CALIFORNIA INSTITUTE OF TECHNOLOGY

PASADENA, CALIFORNIA 91125

ECONOMIES WITH MANY COMMODITIES

C. D. Aliprantis

IUPUI

Kim C. Border

Caltech

Owen Burkinshaw

IUPUI

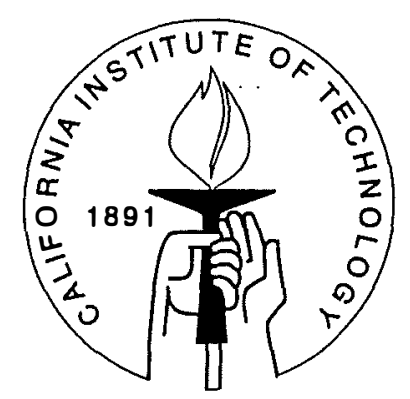

SOCIAL SCIENCE WORKING PAPER 944

October 1995 


\title{
Economies with Many Commodities
}

\author{
C. D. Aliprantis Kim C. Border Owen Burkinshaw
}

\begin{abstract}
We discuss the two fundamental theorems of welfare economics in the context of the Arrow-Debreu-McKenzie model with an infinite dimensional commodity space. As an application, we prove the existence of competitive equilibrium in the standard single agent growth model.
\end{abstract}

JEL classification numbers: 021,111

Key words: General equilibrium, properness, core equivalence, existence 


\title{
Economies with Many Commodities*
}

\author{
C. D. Aliprantis ${ }^{\dagger} \quad$ Kim C. Border ${ }^{\ddagger} \quad$ Owen Burkinshaw ${ }^{\S}$
}

\section{Introduction}

It has become clear in the last couple of decades that in order to address real policy questions, we must employ economic models that are both stochastic and dynamic. These models lead naturally to infinite dimensional commodity spaces. The future is uncertain, and infinitely many resolutions of this uncertainty are conceivable. There is no natural terminal period, so time horizons must be open ended. In this survey, we discuss the problems of working with infinite dimensional spaces in connection with the existence and efficiency of Walrasian equilibria in the Arrow-Debreu-McKenzie framework. To simplify the exposition, we primarily consider pure exchange economies.

The main technical difficulties stem from the following considerations. On a finite dimensional commodity space there is only one natural linear topology, namely the Euclidean topology. In the Euclidean topology every linear functional is continuous. Consequently there is only one natural price space, and it looks just like the commodity space. Furthermore, the value of a commodity vector is a jointly continuous function of the commodity vector and the price vector. On an infinite dimensional commodity space, there is generally more than one natural topology, and the topological dual, the set of continuous linear functionals, depends on the topology. Many of these topologies, especially the useful ones, are not described by metrics. The dual space may not have an interpretation in terms of prices of individual commodities. Furthermore, the value of a commodity vector is not necessarily a jointly continuous function of the price and commodity vectors.

In finite dimensional Euclidean spaces, every closed and bounded set is compact, so continuous functions and preferences have greatest elements. In infinite dimensional

*The research of C. D. Aliprantis was supported in part by the NATO Collaborative Research Grant \# 941059. USA

$\dagger$ Department of Mathematical Sciences, IUPUI, 402 N. Blackford Street, Indianapolis, IN 46202-3216,

$\ddagger$ Division of the Humanities and Social Sciences, 228-77 Caltech, Pasadena, CA 91125, USA

$\S$ Department of Mathematical Sciences, IUPUI, 402 N. Blackford Street, Indianapolis, IN 46202-3216, USA 
spaces, closed and bounded sets need not be compact, and compact sets are rather hard to come by. The choice of topology determines the number of compact sets, but the tradeoff is generally that any topology that admits a lot of compact sets admits very few continuous functions.

In finite dimensional spaces, disjoint nonempty convex sets can always be separated by a hyperplane. In infinite dimensional spaces this is not true. Either one of the sets needs to have a nonempty interior, or both must be closed with one compact. While nonempty interiors may seem natural in the finite dimensional case, in many of the topologies economists use, the set of positive vectors for.instance may. have an empty interior.

Finally, in the finite dimensional case, monotonicity of preferences with respect to the natural order structure of the commodity space plays a marginal role. This assumption can easily be replaced by local nonsatiation conditions and conditions on asymptotic cones of consumption and production sets. In the infinite dimensional case, the order structure plays an important role. In particular, the natural order structure on the price space appears at this time to be crucial.

There are four broad approaches that characterize most proofs of the existence of Walrasian equilibrium in the case of a finite dimensional commodity space. The first and perhaps most natural is the supply and demand approach. This approach shows that an equilibrium (more accurately a quasiequilibrium) exists by showing that the process of raising the price of commodities with excess demand and lowering the price of those in excess supply has a fixed point. That is, there is a price vector that requires no adjustments. Examples of this approach include [82, 83, 161, 163, 169]. The difficulties of generalizing this approach to infinite dimensional spaces comes from the fact that budget sets are not generally compact, so that demand is not well defined. This can be remedied by looking at an increasing sequence of compact sets, finding a market clearing price for the "compactified" economy, and taking a limit of the prices. This works fine in the finite dimensional case, but in the infinite dimensional case, the sequence of prices may well converge to zero. There are ways however to adapt this approach to infinite dimensional settings, see for instance $[97,210]$.

A second approach might be called the noncooperative generalized game theoretic approach. Consumers and producers are viewed as players in a game where there strategy space consists of commodity vectors that correspond to consumption or production plans. Unlike normal form games, the set of feasible strategies is not fixed, but depends on the strategy choices of other players. A fictitious player called the auctioneer is introduced, whose strategy space is the price space. Consumers' payoffs are their utilities, producers' payoffs are their profits, and the auctioneer's payoff is the value of excess demand. This approach then uses a generalization of the existence theorem for Nash equilibrium to show the existence of Walrasian equilibrium. This approach has been especially useful for models where the consumers' preferences are intransitive. Examples of this approach include $[27,78,104,105,193]$. This approach has the same difficulties as the supply and 
demand approach for infinite dimensional economies, but see, for instance [99, 220].

A third approach might be called the welfare based approach. It relies on the Pareto efficiency of Walrasian equilibria and the second welfare theorem. When there are $m$ consumers, the set of Pareto efficient allocations is homeomorphic to the $m-1$ simplex [74]. Given a Pareto efficient allocation, there is a price vector that supports the allocation. At that price vector consumer budgets may not balance, so the allocation is adjusted by lowering their utility level or welfare weight of consumers who are exceeding their budgets. Standard fixed point arguments show that this process has a fixed point. That is, there is an allocation that requires no adjustment to balance the budgets. .This approach was pioneered by Negishi [167] and adopted by Arrow and Hahn [28] and Mas-Colell [152]. Since all the adjustments take place in the $m-1$ simplex, the difficulties for the infinite dimensional case all revolve around the second welfare theorem.

The fourth approach, which we might call the Edgeworth equilibrium approach, is a refinement of the welfare approach. It relies on the core convergence result that the core of a replica economy "shrinks" to the set of Walrasian equilibria. Given that the core of an economy is nonempty and compact (in the finite dimensional world), the intersection of the cores of the replica economies is also nonempty and compact. Debreu and Scarf [87] noted that this approach could be used to prove the existence of equilibrium. The nonemptiness theorem for the core is again a finite dimensional result, relying in fixed point theorems in the utility space $\mathbb{R}^{m}$ for $m$ players. This approach is used in $[5,6,9]$.

Not all proofs of the existence of equilibrium fall neatly into one of these categories, a notable exception being the insightful paper of D. Luenberger [138].

Here is a brief review of the organization and the material presented in this article. We start by presenting some fundamental concepts from the theory of Riesz spaces. Then we discuss preferences and utility functions and describe the characteristics of exchange economies. Subsequently, we discuss the notion of Pareto efficiency and the welfare theorems. Next, the notion of properness is introduced followed by a discussion of the validity of the welfare theorems in the presence of properness. The notion of a Walrasian equilibrium is discussed next followed by a complete investigation of the existence of Walrasian equilibria. In addition to the notion of a Walrasian equilibrium we introduce the core and Edgeworth equilibria and discuss in detail their relationships. As an application of the theory presented here, we formulate and prove the existence of a competitive equilibrium in the single agent growth model.

\section{Riesz spaces}

The natural framework for infinite dimensional commodity spaces seems to be Riesz spaces, so a few definitions are in order. (For a fuller exposition of these concepts see [2, 10, 11].) A Riesz space is a vector space that is equipped with a partial order $\leqslant$ that makes it a lattice. A partial order is a transitive, reflexive, and antisymmetric binary 
relation. (We write $x \geqslant y$ to mean $y \leqslant x$, and $x>y$ means $x \geqslant y$ and $x \neq y$.) For instance, the pointwise order on a space of functions is a partial order. A lattice is a set with a partial order having the property that each pair $x, y$ has both a supremum (least upper bound) $x \vee y$ and an infimum (greatest lower bound) $x \wedge y$. A vector $x$ is positive if $x \geqslant 0$. The cone of positive vectors in a Riesz space $E$ is denote $E^{+}$or $E_{+}$. The absolute value of a vector $x$ in a Riesz space is defined by $|x|=x \vee(-x)$. A subset $A$ of a Riesz space is solid if $|x| \leqslant|y|$ and $y \in A$ imply $x \in A$. A Riesz subspace of a Riesz space $E$ is a vector subspace that is closed under the lattice operations. A solid Riesz subspace of a Riesz space $E$ is called an ideal in $E$. For $z \in E^{+}$, then the principal ideal (or simply the ideal) $E_{z}$ generated by $z$ is the vector subspace

$$
E_{z}=\{x \in E: \exists \lambda>0 \text { such that }|x| \leqslant \lambda z\} .
$$

For example, the set $C[0,1]$ of continuous functions on the unit interval is a lattice under the pointwise order. The supremum of $f$ and $g$ is given by $f \vee g(x)=\max \{f(x), g(x)\}$, which is continuous. On the other hand, $C^{1}(0,1)$, the vector space of continuously differentiable functions on $(0,1)$ is not a lattice under the pointwise ordering. For instance, the pair of functions $f(x)=x$ and $g(x)=1-x$ has no least upper bound in $C^{1}(0,1)$. (There are plenty of upper bounds, but no continuously differentiable upper bound is smallest. The smallest continuous function that is pointwise as large as both is not differentiable, it has a kink at $\frac{1}{2}$.)

A linear functional $p: E \rightarrow \mathbb{R}$ on a Riesz space $E$ is positive if $x \geqslant 0$ implies $p(x) \geqslant 0$. The vector space consisting of the differences of all pairs of positive linear functionals is called the order dual of $E$ and is denoted $E^{\sim}$. It turns out that the order dual $E^{\sim}$ is a Riesz space under the pointwise order. If $p, q \in E^{\sim}$, then $p \vee q$ and $p \wedge q$ are given by

$$
p \vee q(x)=\sup \{p(y)+q(z): y, z \geqslant 0 \text { and } y+z=x\}
$$

and

$$
p \wedge q(x)=\inf \{p(y)+q(z): y, z \geqslant 0 \text { and } y+z=x\}
$$

for each $x \geqslant 0$.

In addition to the order structure, we also require a topological structure in order to carry out any economic analysis. A topology is a means for defining continuity and convergence. (See for instance [2].) A linear topology on a vector space is a topology for which vector addition and scalar multiplication are continuous functions. A typical (but no means the most useful) way to define a topology on a vector space is by means of a norm. A norm $\|\cdot\|$ is a real-valued function on a vector space satisfying: (1) $\|x\| \geqslant 0$ for all $x$. (2) $\|x\|=0$ implies $x=0$. (3) $\|x+y\| \leqslant\|x\|+\|y\|$. A lattice norm on a Riesz space is a norm such that $|x| \leqslant|y|$ implies $\|x\| \leqslant\|y\|$ ). (A norm $\|\cdot\|$ defines a topology through the metric $d$ defined by $d(x, y)=\|x-y\|$.)

As a matter of fact the most commonly employed vector spaces in economics are all either Banach lattices or topological Riesz spaces. A Banach lattice is a Riesz 
space which is a Banach space under a lattice norm. A topological Riesz space is a Riesz space equipped with a linear topology which is also locally solid, that is, it has a neighborhood base at zero consisting of solid sets.

A dual pair a pair $\left\langle X, X^{\prime}\right\rangle$ of vector spaces and an associated bilinear functional $\langle\cdot, \cdot\rangle: X \times X^{\prime} \rightarrow \mathbb{R}$ that separates points, called the duality of the pair. Thus $X$ can be regarded as a vector space of linear functionals on $X^{\prime}$, and vice versa, via the relation $x\left(x^{\prime}\right)=\left\langle x, x^{\prime}\right\rangle=x^{\prime}(x)$. A topology $\tau$ on $X$ is consistent with the dual pair if every functional in $X^{\prime}$ is $\tau$-continuous. Likewise, a topology $\tau^{\prime}$ on $X^{\prime}$ is consistent if every functional in $X$ is $\tau^{\prime}$-continuous. Given a dual pair, the weak topology on $X, \sigma\left(X, X^{\prime}\right)$, is the weakest consistent topology on $X$. The weak* topology on $X^{\prime}$, $\sigma\left(X^{\prime}, X\right)$, is the weakest consistent topology on $X^{\prime}$. The Mackey-Arens Theorem [2, Theorem 4.86] asserts that there is also strongest consistent topology on $X$, called the Mackey topology, denoted $\tau\left(X, X^{\prime}\right)$. See [2, Sections 4.13-4.17] for a fuller explanation of dual pairs.

We employ two kinds of dual pairs closely associated with lattice properties-Riesz pairs and lattice pairs. A Riesz pair $\left\langle E, E^{\prime}\right\rangle$ is a dual pair with the property that $E$ is a Riesz space (vector lattice) and $E^{\prime}$ is an ideal in the order dual $E^{\sim}$ that separates the points of $E$. In particular, the dual pair $\left\langle E, E^{\sim}\right\rangle$ is a Riesz pair, where $E$ is a Riesz space and $E^{\sim}$ is its order dual (provided that $E^{\sim}$ separates the points of $E$ ). A lattice pair $\left\langle E, E^{\prime}\right\rangle$ is a dual pair such that $E$ is a Riesz space and $E^{\prime}$ is a Riesz subspace of the order dual $E^{\sim}$ that separates the points of $E$. Clearly, every Riesz pair is a lattice pair, but the converse is false; $\langle c a[0,1], C[0,1]\rangle$ is a lattice pair but not a Riesz pair. A Riesz pair $\left\langle E, E^{\prime}\right\rangle$ is symmetric if $E$ is an ideal in $\left(E^{\prime}\right)^{\sim}$ or, equivalently, if $\left\langle E^{\prime}, E\right\rangle$ is also a Riesz pair. Here are two useful properties of Riesz pairs.

a. A Riesz pair $\left\langle E, E^{\prime}\right\rangle$ is symmetric if and only if the order intervals of $E$ are $\sigma\left(E, E^{\prime}\right)$ weakly compact.

b. If $\left\langle E, E^{\prime}\right\rangle$ is a Riesz pair, then $\left\langle E^{\prime}, E\right\rangle$ is a lattice pair with the property that each order interval of $E^{\prime}$ is $\sigma\left(E^{\prime}, E\right)$-compact [2, Lemma 6.53].

If $\left\langle E, E^{\prime}\right\rangle$ is a lattice pair, then an element $x \in E^{+}$is strictly positive, written $x \gg 0$, if $\left\langle x, x^{\prime}\right\rangle>0$ for each $0<x^{\prime} \in E^{\prime}$. If $E=\mathbb{R}^{\ell}$, then a positive vector is strictly positive if and only if each component of $x$ is strictly positive. Similarly, a vector $0<x^{\prime} \in E^{\prime}$ is strictly positive if $0<x \in E^{+}$implies $\left\langle x, x^{\prime}\right\rangle>0$.

For more about Riesz pairs see [11] and [2, Sections 6.15 and 6.16]. We are now ready to classify economies according to the lattice properties of the commodity and price spaces.

\section{Preferences and utility functions}

A preference $\succcurlyeq$ on a set $X$ is a binary relation on $X$ that is 
1. reflexive: $x \succcurlyeq x$ for all $x \in X$,

2. transitive: $x \succcurlyeq y$ and $y \succcurlyeq z$ imply $x \succcurlyeq z$, and

3. total: for each $x, y \in X$ with $x \neq y$ either $x \succcurlyeq y$ or $y \succcurlyeq x$.

The expression $x \succcurlyeq y$ means $x$ is as good as $y$. When both $x \succcurlyeq y$ and $y \succcurlyeq x$, then $x$ is indifferent to $y$, written $x \sim y$. In case both $x \succcurlyeq y$ and $y \nsucceq x$, we say that $x$ is strictly preferred to $y$, denoted $x \succ y$.

Definition 1 Let $\succcurlyeq$ be a preference on a subset $X$ of a vector space. . A vector $v$, not necessarily lying in $X$, is desirable (for $\succcurlyeq$ ) if for each $x \in X$ there exists some $\delta>0$ such that for each $0<\alpha<\delta$ we have $x+\alpha v \in X$ and $x+\alpha v \succ x$.

We now introduce some important topological, algebraic, and order properties of preference relations. A preference relation $\succcurlyeq$ on a topological space $X$ is:

- upper semicontinuous if the upper contour set $\{y \in X: y \succcurlyeq x\}$ is closed for each $x \in X$.

- lower semicontinuous if the lower contour set $\{z \in X: x \succcurlyeq z\}$ is closed for each $x \in X$.

- continuous if $\succcurlyeq$ is both upper and lower semicontinuous.

When $X$ is a convex subset of a vector space, a preference relation $\succcurlyeq$ on $X$ is:

- convex if the upper contour set $\{y \in X: y \succcurlyeq x\}$ is convex for each $x \in X$.

- strictly convex if $y \succcurlyeq x, z \succcurlyeq x, y \neq z$, and $0<\alpha<1$ imply $\alpha y+(1-\alpha) z \succ x$.

In case the opportunity set $X$ is partially ordered by $\geqslant$, then we say that a preference relation $\succcurlyeq$ on $X$ is:

- monotone (with respect to $\geqslant$ ) if $x \geqslant y$ implies $x \succcurlyeq y$.

- strictly monotone if $x>y$ implies $x \succ y$.

An element $a \in X$ is maximal for a binary relation $\succcurlyeq$ on a set $X$ if there is no other element $x \in X$ with $x \succ a$. When $\succcurlyeq$ is total and reflexive this is equivalent to $a$ being a greatest element, i.e., $a \succcurlyeq x$ for all $x \in X$. The next theorem deals with the properties and existence of greatest elements for preference relations.

Theorem 2 For a preference $\succcurlyeq$ on a set $X$ :

1. Greatest elements are indifferent to each other. 
2. If $X$ is a compact space and $\succcurlyeq$ is upper semicontinuous, then $\succcurlyeq$ has greatest elements.

3. If $X$ is convex and $\succcurlyeq$ is a convex preference, then the (possibly empty) set of greatest elements of $\succcurlyeq$ is convex.

4. If $X$ is convex and $\succcurlyeq$ is strictly convex, then $\succcurlyeq$ has at most one greatest element.

A utility function for a preference relation $\succcurlyeq$ is a function $u: X \rightarrow \mathbb{R}$ such that for every $x$ and $y, u(x) \geqslant u(y)$ if and only if $x \succcurlyeq y$. In this case we say $u$ represents $\succcurlyeq$ on $X$. Every real function $u: X \rightarrow \mathbb{R}$ gives rise to a preference relation $\succcurlyeq$ defined by $x \succcurlyeq y$ if $u(x) \geqslant u(y)$. Note that a utility function is quasiconcave if and only it represents a convex preference.

It is well known that even for finite dimensional commodity spaces, not every preference is representable by a utility function. For instance, the lexicopgraphic ordering of the plane has no utility. Additional assumptions, typically (semi)continuity assumptions, are needed for the existence of a representation. G. Debreu $[79,82]$ proves the existence of a continuous utility for a continuous preference relation on connected subsets of $\mathbb{R}^{n}$. T. Rader [181] and M. K. Richter [187] give conditions guaranteeing the existence of an upper semicontinuous for an upper semicontinuous preference. Debreu's proof makes use of the separability of $\mathbb{R}^{n}$ and the Rader-Richter approach uses second countability of $\mathbb{R}^{n}$. Unfortunately, infinite dimensional Riesz spaces do not possess either property. Nonetheless, S. Richard and W. Zame [186] prove that uniformly proper, continuous, and convex preferences on the positive cone of a normed vector lattice have a representation by a continuous utility function. Their construction involves the fact that for uniformly proper preferences, there is a ray crossed by every indifference curve. Since each point on the ray can be written as a positive multiple $\alpha$ of some fixed vector $v$, the value of $\alpha$ where the indifference curve crosses the ray serves as a utility function with the desired properties. They also show that uniform properness is almost a necessary condition for such a representation.

\section{Exchange economies}

One of the basic economic constructions associated with any market environment is the commodity-price duality. G. Debreu [80] was the first to express this in terms of a dual pair $\left\langle X, X^{\prime}\right\rangle$. The vector space $X$ is the commodity space and its vectors are known as commodity bundles (or commodities) and $X^{\prime}$ is the price space whose vectors are called prices. ${ }^{1}$ The real number $\left\langle x, x^{\prime}\right\rangle$ is the value of the bundle $x$ at prices $x^{\prime}$. It is customary in economic theory to designate the price vectors by $p, q$, etc., instead of using primes. Also, the evaluation $\langle x, p\rangle$ is denoted $p \cdot x$, i.e., $p \cdot x=\langle x, p\rangle$. An exchange

\footnotetext{
${ }^{1}$ Debreu [80] used the term valuation for elements in the dual and reserved the term price system for valuations which could be represented as integrals.
} 
economy consists of a set of consumers who exchange goods but are unable to produce anything other than their endowment. More formally:

Definition $3 A$ (private ownership) exchange economy is a tuple

$$
\mathcal{E}=\left(\left\langle X, X^{\prime}\right\rangle,\left(X_{1}, \succcurlyeq_{1}, \omega_{1}\right), \ldots,\left(X_{m}, \succcurlyeq_{m}, \omega_{m}\right)\right)
$$

where:

- The commodity-price duality is described by the dual pair $\left\langle X, X^{\prime}\right\rangle ; X$ is the commodity space and $X^{\prime}$ is the price space.

- The economy has $m$ consumers. Each consumer $i$ has a consumption set $X_{i} \subset X$ and an initial endowment $\omega_{i} \in X_{i}$. The vector $\omega=\sum_{i=1}^{m} \omega_{i}$ is known as the total endowment or the social endowment.

- The tastes of each consumer $i$ are described by his preference relation $\succcurlyeq_{i}$ on $X_{i}$.

An allocation for any such economy is simply an $m$-tuple $\left(x_{1}, \ldots, x_{m}\right)$ of vectors such that $x_{i} \in X_{i}$ for each $i$ and $\sum_{i=1}^{m} x_{i}=\omega$. In other words, allocations are redistributions of the social endowment amongst the $m$ consumers of the economy. When $X$ is an ordered space, we refer to the order interval $[0, \omega]=\{x \in X: 0 \leqslant x \leqslant \omega\}$ as the Edgeworth box of the exchange economy.

Definition 4 An economy is called:

- A Riesz economy if its commodity-price duality is described by a Riesz pair $\left\langle E, E^{\prime}\right\rangle$ and each consumer's consumption set is $E^{+}$.

- A lattice economy if its commodity-price duality is described by a lattice pair $\left\langle E, E^{\prime}\right\rangle$ and each consumer's consumption set is $E^{+}$.

- A Riesz commodity economy if its commodity space is a Riesz space (in which case we denote the commodity-price dual pair by $\left.\left\langle E, X^{\prime}\right\rangle\right)$ and each consumer's consumption set is $E^{+}$.

- A Riesz price economy if its price space is a Riesz space (in which case we denote the commodity-price dual pair by $\left.\left\langle X, E^{\prime}\right\rangle\right)$.

- A convex economy if all preferences and consumption sets are convex.

- A finite dimensional economy if it is a Riesz economy with Riesz pair $\left\langle\mathbb{R}^{\ell}, \mathbb{R}^{\ell}\right\rangle$ and the total endowment is strictly positive. 


\section{$5 \quad$ Efficiency}

The collection of allocations is denoted $\mathcal{A}$, i.e.,

$$
\mathcal{A}=\left\{\left(x_{1}, \ldots, x_{m}\right): x_{i} \in X_{i} \text { for each } i \text { and } \sum_{i=1}^{m} x_{i}=\omega\right\}
$$

Clearly, $\left(\omega_{1}, \ldots, \omega_{m}\right) \in \mathcal{A}$, so $\mathcal{A}$ is a nonempty set. The fundamental value judgement made in neoclassical economics is that consumers' preferences should be used to evaluate their welfare. This leads naturally to the following notions.

Definition 5 An allocation $\left(x_{1}, \ldots, x_{m}\right)$ is:

- Pareto efficient if there is no allocation $\left(z_{1}, \ldots, z_{m}\right)$ satisfying $z_{i} \succcurlyeq_{i} x_{i}$ for all $i$ and $z_{s} \succ_{s} x_{s}$ for some $s$.

- weakly Pareto efficient if there is no allocation $\left(z_{1}, \ldots, z_{m}\right)$ with $z_{i} \succ_{i} x_{i}$ for all $i$.

- individually rational if $x_{i} \succcurlyeq_{i} \omega_{i}$ for each $i$.

The set of individually rational allocations is denoted $\mathcal{A}_{r}$, i.e.,

$$
\mathcal{A}_{r}=\left\{\left(x_{1}, \ldots, x_{m}\right) \in \mathcal{A}: x_{i} \succcurlyeq_{i} \omega_{i} \text { for each } i\right\}
$$

Likewise, the set of weakly Pareto efficient allocations is denoted $\mathcal{A}_{w p}$, i.e., $\mathcal{A}_{w p}=\left\{\left(x_{1}, \ldots, x_{m}\right) \in \mathcal{A}:\right.$ There is no $\left(y_{1}, \ldots, y_{m}\right) \in \mathcal{A}$ satisfying $y_{i} \succ_{i} x_{i}$ for each $\left.i\right\}$.

Of course, every Pareto efficient allocation is weakly Pareto efficient. But the converse is false; see Example 33 below. However, with strictly monotone continuous preferences the notions of Pareto and weak Pareto efficiency coincide.

Lemma 6 In a Riesz commodity exchange economy, if each preference is strictly monotone and lower semicontinuous for some linear topology, then every weakly Pareto efficient allocation is Pareto efficient.

An obvious question is: Do individually rational Pareto efficient allocations exist? Before answering this:question (affirmatively), we characterize the compactness of $\mathcal{A}$.

Theorem 7 In a Riesz commodity exchange economy with weakly closed Edgeworth box $[0, \omega]$ and at least two consumers, the set $\mathcal{A}$ of allocations is weakly compact in $E^{m}$ (that is, $\sigma\left(E^{m},\left(X^{\prime}\right)^{m}\right)$-compact) if and only if the Edgeworth box $[0, \omega]$ is weakly compact (i.e., $\sigma\left(E, X^{\prime}\right)$-compact). 
Proof: Notice that $\mathcal{A} \subset[0, \omega]^{m}$. In addition, since $\sigma\left(E^{m},\left(X^{\prime}\right)^{m}\right)=\prod_{i=1}^{m} \sigma\left(E, X^{\prime}\right)$, it is easy to see that $\mathcal{A}$ is a $\sigma\left(E^{m},\left(X^{\prime}\right)^{m}\right)$-closed subset of $E^{m}$. Now suppose that the Edgeworth box $[0, \omega]$ is $\sigma\left(E, X^{\prime}\right)$-compact. Then $[0, \omega]^{m}$ is $\sigma\left(E^{m},\left(X^{\prime}\right)^{m}\right)$-compact, so the $\sigma\left(E^{m},\left(X^{\prime}\right)^{m}\right)$-closed subset $\mathcal{A}$ of $[0, \omega]^{m}$ is likewise $\sigma\left(E^{m},\left(X^{\prime}\right)^{m}\right)$-compact. For the converse, assume that $\mathcal{A}$ is a $\sigma\left(E^{m},\left(X^{\prime}\right)^{m}\right)$-compact subset of $E^{m}$ and let $\left\{x_{\alpha}\right\}$ be a net in $[0, \omega]$. Put

$$
y_{\alpha}=\left(x_{\alpha}, \omega-x_{\alpha}, 0,0, \ldots, 0\right) .
$$

and note that $\left\{y_{\alpha}\right\}$ is a net in $\mathcal{A}$. Hence, $\left\{y_{\alpha}\right\}$ has a $\sigma\left(E^{m},\left(X^{\prime}\right)^{m}\right)$-convergent subnet in $E^{m}$, and from this it follows that $\left\{x_{\alpha}\right\}$ also has a $\sigma\left(E, X^{\prime}\right)$-convergent subnet in $[0, \omega]$. Consequently, the Edgeworth box $[0, \omega]$ is $\sigma\left(E, X^{\prime}\right)$-compact.

Theorem 8 In a Riesz commodity exchange economy, if the Edgeworth box $[0, \omega]$ is weakly compact and preferences are weakly upper semicontinuous, then the nonempty sets $\mathcal{A}_{r}$ (of all individually rational allocations) and $\mathcal{A}_{\text {wp }}$ (of weakly Pareto efficient allocations) are both weakly compact.

Proof: In this case, both $\mathcal{A}_{r}$ and $\mathcal{A}_{w p}$ are weakly closed subsets of the weakly compact set $\mathcal{A}$.

Relatively mild conditions guarantee the existence of individually rational Pareto efficient allocations.

Theorem 9 In a Riesz commodity exchange economy, if the Edgeworth box $[0, \omega]$ is weakly compact and preferences are weakly upper semicontinuous, then individually rational Pareto efficient allocations exist.

Proof: Start by introducing on $\mathcal{A}_{r}$ the equivalence relation $\sim$ by saying that

$$
\left(x_{1}, \ldots, x_{m}\right) \sim\left(z_{1}, \ldots, z_{m}\right)
$$

if $x_{i} \sim_{i} z_{i}$ for each $i$. Notice that $\sim$ is indeed an equivalence relation on $\mathcal{A}_{r}$. For simplicity, denote the set of all equivalence classes $\mathcal{A}_{r} / \sim$ by $\mathcal{A}_{r}$ again. Next, we define an ordering $\geqslant$ on (the equivalence classes) $\mathcal{A}_{r}$ by

$$
x=\left(x_{1}, \ldots, x_{m}\right) \geqslant z=\left(z_{1}, \ldots, z_{m}\right)
$$

if $x_{i} \succcurlyeq_{i} z_{i}$ for each $i$. Again, notice that $\geqslant$ is a partial order on $\mathcal{A}_{r}$ and that the maximal elements of $\geqslant$ are precisely the Pareto efficient allocations. So in order to establish that Pareto efficient allocations exist, it suffices to show that $\geqslant$ has maximal elements. For this, we need to establish that the partially ordered set $\left(\mathcal{A}_{r}, \geqslant\right)$ satisfies the hypothesis of Zorn's lemma. That is, we must show that every chain of $\mathcal{A}_{r}$ has an upper bound.

To this end, let $\mathcal{C}$ be a chain in $\mathcal{A}_{r}$, that is, for each pair $x, z \in \mathcal{C}$ either $x \geqslant z$ or $z \geqslant x$. Put $x_{\alpha}=\alpha$ for each $\alpha \in \mathcal{C}$ and note that $\left\{x_{\alpha}\right\}_{\alpha \in \mathcal{C}}$ is a net in the weakly compact set $\mathcal{A}_{r}$. If $x \in \mathcal{A}_{r}$ is a limit point of the net $\left\{x_{\alpha}\right\}_{\alpha \in \mathcal{C}}$, then it follows from the weak upper semicontinuity of the preferences that $x \geqslant y$ for each $y \in \mathcal{C}$, as desired. 
Corollary 10 In a Riesz commodity exchange economy, if the Edgeworth box $[0, \omega]$ is weakly compact and each preference is convex and continuous with respect to a consistent locally convex topology on $E$, then individually rational Pareto efficient allocations exist.

Proof: Notice that in this case every preference is weakly upper semicontinuous.

\section{Efficiency, prices, and the welfare theorems}

We are now ready to discuss efficiency properties of allocations in the presence of prices. As we shall see, the Pareto efficiency of an allocation can be characterized in terms of its support by prices. We start with the definition of a price-supported allocation.

Definition 11 An allocation $\left(x_{1}, \ldots, x_{m}\right)$ in an exchange economy is supported by prices if there exists a nonzero price $p \in X^{\prime}$ (called a supporting price) such that

$$
x \succcurlyeq_{i} x_{i} \text { in } X_{i} \text { implies } p \cdot x \geqslant p \cdot x_{i} .
$$

In Riesz commodity exchange economies supporting prices are positive.

Lemma 12 In a Riesz commodity exchange economy, if at least one consumer has a monotone preference, then every supporting price is positive.

Proof: Let the first consumer has a monotone preference and let $\left(x_{1}, \ldots, x_{m}\right)$ be an allocation supported by a nonzero price $p$. If $x \geqslant 0$, then $x+x_{1} \succcurlyeq_{1} x_{1}$, and since $p$ is a supporting price, we infer that $p \cdot\left(x+x_{1}\right) \geqslant p \cdot x_{1}$. This inequality implies $p \cdot x \geqslant 0$, which means that $p \geqslant 0$.

The objective of this section is to establish versions of the following efficiency properties of allocations known as the first and second welfare theorems. These theorems were first proven in reasonable generality by K. Arrow [25] and G. Debreu [77, 80], although I. Fisher in the 1890s and V. Pareto and O. Lange in the 1920s knew special cases. The two theorems can be stated (subject to appropriate conditions) as:

- First Welfare Theorem: Every allocation supported by prices is Pareto efficient.

- Second Welfare Theorem: Every Pareto efficient allocation can by supported by prices.

Theorem 13 (First Welfare Theorem) In a lattice exchange economy suppose that:

1. Each preference is continuous for some linear topology;

2. The total endowment is strictly positive (i.e., $\omega \gg 0$ ); and 
3. At least one consumer has a monotone preference.

Then every allocation supported by prices is weakly Pareto efficient.

Proof: Given the hypotheses, let $\left(x_{1}, \ldots, x_{m}\right)$ be an allocation supported by a price $p$. By Lemma 12, we have $p>0$. Suppose by way of contradiction that $\left(x_{1}, \ldots, x_{m}\right)$ is not weakly Pareto efficient. Then there exists another allocation $\left(z_{1}, \ldots, z_{m}\right)$ with $z_{i} \succ_{i} x_{i}$ for each $i$. Since $p$ is supporting, we infer that $p \cdot z_{i} \geqslant p \cdot x_{i}$ for each $i$, and from the identity

$$
\sum_{i=1}^{m} p \cdot z_{i}=p \cdot\left(\sum_{i=1}^{m} z_{i}\right)=p \cdot \omega=p \cdot\left(\sum_{i=1}^{m} x_{i}\right)=\sum_{i=1}^{m} p \cdot x_{i},
$$

we see that $p \cdot z_{i}=p \cdot x_{i}$ for each $i$. Now from $\omega=\sum_{i=1}^{m} x_{i} \gg 0$ and $p>0$, we have $p \cdot \omega=\sum_{i=1}^{m} p \cdot x_{i}>0$. In particular, there exists some $s$ with $p \cdot x_{s}>0$. Since $\succcurlyeq_{s}$ is continuous for some linear topology, there exists some $0<\delta<1$ such that $\delta z_{s} \succ_{s} x_{s}$, and since $p$ is supporting, we obtain $p \cdot\left(\delta z_{s}\right) \geqslant p \cdot x_{s}$. However, we also have

$$
p \cdot\left(\delta z_{s}\right)=\delta p \cdot z_{s}=\delta p \cdot x_{s}<p \cdot x_{s},
$$

which is a contradiction. Hence, the allocation $\left(x_{1}, \ldots, x_{m}\right)$ must be weakly Pareto efficient.

In finite dimensional economies, it is relatively easy to establish the second welfare theorem too.

Theorem 14 (Second Welfare Theorem) If a finite dimensional exchange economy is convex and the total endowment is desirable for each consumer, then every weakly Pareto efficient (and hence every Pareto efficient) allocation can be supported by prices.

The infinite dimensional analogue of the Second Welfare Theorem 14 may fail, as we can see from the following example.

Example 15 (Aliprantis-Burkinshaw [12]) Consider a Riesz exchange economy with two consumers having the following characteristics:

- Commodity-price duality the symmetric Riesz pair $\left\langle\ell_{1}, \ell_{\infty}\right\rangle$.

- Initial endowments $\omega_{1}=\omega_{2}=\left(\frac{1}{2^{3}}, \frac{1}{2^{5}}, \ldots, \frac{1}{2^{2 n+1}}, \ldots\right)$.

- Preferences for both agents are represented on $\ell_{1}^{+}$by the utility function

$$
u(x)=\sum_{n=1}^{\infty} \varphi_{n}\left(x_{n}\right)
$$

where the functions $\varphi_{n}: \mathbb{R} \rightarrow \mathbb{R}$ are defined by

$$
\varphi_{n}(t)= \begin{cases}2^{n} t, & \text { if } t \leqslant \frac{1}{2^{2 n}} \\ \frac{1}{2^{n}}-\frac{1}{2^{2 n}}+t, & \text { if } t>\frac{1}{2^{2 n}}\end{cases}
$$


This utility was introduced by A. Mas-Colell [152]. Since $t \geqslant 0$ implies $0 \leqslant \varphi_{n}(t) \leqslant \frac{1}{2^{n}}+t$, notice that the series defining $u$ converges for each $x=\left(x_{1}, x_{2}, \ldots\right) \in \ell_{1}^{+}$.

A direct verification shows that the utility function is concave, strictly monotone, and norm continuous. In addition, note that the total endowment $\omega=\omega_{1}+\omega_{2}$ is strictly positive. We now claim that the allocation $\left(\omega_{1}, \omega_{2}\right)$ is Pareto efficient (hence weakly Pareto efficient) but fails to be supported by prices.

We establish first that $\left(\omega_{1}, \omega_{2}\right)$ is Pareto efficient. To see this, start by observing that if $a=\left(a_{1}, a_{2}, \ldots\right)$ and $b=\left(b_{1}, b_{2}, \ldots\right)$ are two arbitrary positive sequences satisfying $0 \leqslant a_{n}+b_{n} \leqslant 2^{-2 n}$ for all $n$, then

$$
u(a+b)=u(a)+u(b) .
$$

Next, let $\left(x_{1}, x_{2}\right)$ be an allocation satisfying $x_{1} \succcurlyeq \omega_{1}$ and $x_{2} \succcurlyeq \omega_{2}$. From $x_{1}+x_{2}=\omega_{1}+\omega_{2}$ and the above observation, we see that

$$
u\left(x_{1}\right)+u\left(x_{2}\right)=u\left(x_{1}+x_{2}\right)=u\left(\omega_{1}+\omega_{2}\right)=u\left(\omega_{1}\right)+u\left(\omega_{2}\right) .
$$

From $u\left(x_{1}\right) \geqslant u\left(\omega_{1}\right)$ and $u\left(x_{2}\right) \geqslant u\left(\omega_{2}\right)$, we infer that $u\left(x_{1}\right)=u\left(\omega_{1}\right)$ and $u\left(x_{2}\right)=u\left(\omega_{2}\right)$. This implies that the allocation $\left(\omega_{1}, \omega_{2}\right)$ is Pareto efficient.

Finally, we establish that the allocation $\left(\omega_{1}, \omega_{2}\right)$ cannot be supported by prices. To this end, let $p \in \ell_{\infty}$ be a price that supports $\left(\omega_{1}, \omega_{2}\right)$. By the monotonicity of the utility function $u$, it follows that $p \geqslant 0$. Also, an easy computation shows that $\left(1-2^{-k}\right) \omega_{1}+$ $2^{-2 k} e_{k} \succ \omega_{1}$, where $e_{k}$ denotes the sequence whose $k^{\text {th }}$ component is one and every other is equal to zero. Since $p$ is supporting, we get

$$
\left(1-2^{-k}\right) p \cdot \omega_{1}+2^{-2 k} p \cdot e_{k} \geqslant p \cdot \omega_{1} .
$$

Consequently, $0 \leqslant 2^{k} p \cdot \omega_{1} \leqslant p \cdot e_{k} \leqslant\|p\|_{\infty}<\infty$ for all $k$. Thus, $p \cdot \omega_{1}=0$, and since all components of $\omega_{1}$ are positive, we see that $p=0$, which means that $\left(\omega_{1}, \omega_{2}\right)$ cannot be supported by prices.

In order to establish the second welfare theorem in infinite dimensional spaces, we need to add an additional condition on preferences. This property is known as properness and is the subject of the next section.

\section{Properness}

As we saw above, in order to establish the second welfare theorem, we need some additional condition on preferences. This new property was introduced to the economic literature by G. Chichilnisky and P. J. Kalman [72] (see also [70]) as "the cone condition" and was popularized in general equilibrium theory by A. Mas-Colell [152] under the name of "properness." 
Definition 16 (Properness) Let $E$ be a Riesz space, $\tau$ a linear topology on $E, v>0$, and $V$ a $\tau$-neighborhood of zero. A preference relation $\succcurlyeq$ on $E^{+}$is:

1. $(v, V)$-proper at a point $x \in E^{+}$if $x-\alpha v+z \succcurlyeq x$ in $E^{+}$with $\alpha>0$ implies $z \notin \alpha V$,

2. uniformly $(v, V)$-proper if $x-\alpha v+z \succcurlyeq x$ in $E^{+}$with $\alpha>0$ implies $z \notin \alpha V$.

Any vector $v$ that satisfies the second property for some neighborhood $V$ is called a vector of uniform properness for $\succcurlyeq$. When the vector $v$ and the neighborhood $V$ are clear from the context, we simply say that $\succcurlyeq$ is uniformly proper. In case we want to emphasize the vector $v$ only, we say that $\succcurlyeq$ is $v$-uniformly proper. If we wish to emphasize the topology $\tau$, then we say that $\succcurlyeq$ is uniformly $\tau$-proper.

Lemma 17 Vectors of uniform properness are desirable.

Proof: Let $\succcurlyeq$ be a $(v, V)$-uniformly proper preference. Fix $x \in E^{+}$and let $\alpha>0$. If $x \succcurlyeq x+\alpha v$, then from $(x+\alpha v)-\alpha v+0=x \succcurlyeq x+\alpha v$, it follows that $0 \notin \alpha V$, which is impossible. Hence, $x+\alpha v \succ x$ for all $x \in E^{+}$and each $\alpha>0$.

A nonempty open set $\Gamma$ is an open cone if $\alpha x \in \Gamma$ whenever $x \in \Gamma$ and each $\alpha>0$. The next result characterizes uniform properness in terms of open cones.

Theorem 18 (Mas-Colell [152]) Let $\tau$ be a locally convex topology on the Riesz space $E$ and let $\succcurlyeq$ be a preference on $E^{+}$. Then $\succcurlyeq$ is uniformly $\tau$-proper if and only if there exists a non-empty $\tau$-open convex cone $\Gamma$ such that

1. $\Gamma \cap\left(-E^{+}\right) \neq \varnothing ;$ and

2. $(x+\Gamma) \cap\left\{y \in E^{+}: y \succcurlyeq x\right\}=\varnothing$ for all $x \in E^{+}$.

For a proof see $[8$, Theorem 3.2 .3 , p. 117$]$.

The following is a consequence of the preceding result. An AM space with unit is a Banach lattice $E$ where the norm is of the form $\|x\|=\inf \{\lambda>0:|x| \leqslant \lambda e\}$ for some $e$ in $E$.

Theorem 19 (Mas-Colell [152]) If a monotone preference on the positive cone of an AM-space with unit has a.desirable bundle, then it is uniformly norm proper. In particular, every monotone preference on the positive cone of some $\mathbb{R}^{\ell}$ space with a desirable bundle is uniformly proper.

For a proof see $[8$, Theorem 3.2 .4 , p. 118].

Strictly positive vectors are a source of vectors of uniform properness. A locally convex-solid topology on a Riesz space is a topology with a neighborhood base at zero consisting of solid convex sets. 
Lemma 20 Let $\left\langle E, E^{\prime}\right\rangle$ be a Riesz pair and let $\tau$ be a consistent locally convex-solid topology on $E$. If $\succcurlyeq$ is a uniformly $\tau$-proper preference on $E^{+}$, then every strictly positive element of $E$ is a vector of uniform $\tau$-properness for $\succcurlyeq$.

Proof: Let $e$ be a strictly positive vector in $E$. Then the principal ideal $A_{e}$ is weakly (and hence $\tau$ ) dense in $E$; see [2, Theorem 6.51]. Since $\succcurlyeq$ is uniformly $\tau$-proper, there exists a $\tau$-neighborhood $V$ of zero and some $v>0$ such that

$$
x-\alpha v+z \succcurlyeq x \text { in } E^{+} \text {with } \alpha>0 \text { implies } z \notin \alpha V \text {. }
$$

Next, choose a convex and solid $\tau$-neighborhood $W$ of zero with $v \notin W$ and $W+W \subset V$. Pick some $y \in A_{e}$ with $v-y \in W$. Replacing $y$ by $v \wedge y^{+}$and taking into account the inequality $\left|v-\left(v \wedge y^{+}\right)\right| \leqslant|v-y|$, we can suppose without loss of generality that $0<y \leqslant v$. Now note that if $x-\alpha y+z \succcurlyeq x$ in $E^{+}$with $\alpha>0$, then from ( $\star$ ) and the relation

$$
x-\alpha v+[z-\alpha(y-v)]=x-\alpha y+z \succcurlyeq x,
$$

we see that $z-\alpha(y-v) \notin \alpha V$. So if $z \in \alpha W$, then

$$
z-\alpha(y-v) \in \alpha W+\alpha W=\alpha(W+W) \subset \alpha V,
$$

which is a contradiction. The above show that in ( $\star$ ), we can assume (replacing $v$ by $y$ ) that $0<v \in A_{e}$. Now if we pick some $\lambda>0$ such that $0<y \leqslant \lambda e$, then it is easy to see that $\lambda e$ is a vector of uniform $\tau$-properness for $\succcurlyeq$, and consequently $e$ itself is also a vector of uniform $\tau$-properness.

As an application of the preceding result, let us show that Mas-Colell's preference relation $\succcurlyeq$ introduced in Example 15 is not uniformly norm proper.

Corollary 21 The preference relation $\succcurlyeq$ on $\ell_{1}^{+}$represented by the utility function

$$
u\left(x_{1}, x_{2}, \ldots\right)=\sum_{n=1}^{\infty} \varphi_{n}\left(x_{n}\right),
$$

where

$$
\varphi_{n}(t)= \begin{cases}2^{n} t, & \text { if } t \leqslant \frac{1}{2^{2 n}}, \\ \frac{1}{2^{n}}-\frac{1}{2^{2 n}}+t, & \text { if } t>\frac{1}{2^{2 n}},\end{cases}
$$

is not uniformly norm proper.

Proof: To see this, suppose by way of contradiction that $\succcurlyeq$ is uniformly norm proper. Then by Lemma 20, the strictly positive vector $v=\left(\frac{1}{2^{3}}, \frac{1}{2^{5}}, \ldots, \frac{1}{2^{2 n+1}}, \ldots\right)$ must be a vector of uniform norm properness for $\succcurlyeq$. That is, there exists some $\varepsilon>0$ such that $x-\alpha v+z \succcurlyeq x$ in $\ell_{1}^{+}$implies $\|z\|_{1} \geqslant \alpha \varepsilon$. Now note that $v-2^{-n} v+2^{-2 n} e_{n} \succ v$ for each $n$, where $e_{n}$ denotes the sequence having its $\mathrm{n}^{\text {th }}$ component equal to one and every other equal to zero. Hence, $2^{-2 n}\left\|e_{n}\right\|_{1} \geqslant 2^{-n} \varepsilon$ or $1 \geqslant 2^{n} \varepsilon$ for each $n$, which is impossible. Hence $\succcurlyeq$ cannot be uniformly norm proper. 
Consider a dual pair $\left\langle X, X^{\prime}\right\rangle$ and let $\succcurlyeq$ be a preference relation on a subset $S$ of $X$. As usual, we say that a price $p \in X^{\prime}$ supports the preference $\succcurlyeq$ at $x \in S$ if $y \in S$ and $y \succcurlyeq x$ imply $p \cdot y \geqslant p \cdot x$.

If $A$ is a subset of $X$, then its (absolute) polar $A^{\circ}$ is defined by

$$
A^{\circ}=\left\{x^{\prime} \in X^{\prime}:\left|\left\langle x, x^{\prime}\right\rangle\right| \leqslant 1 \text { for all } x \in A\right\} .
$$

The classical Alaoglu theorem [2, Theorem 4.79, p. 158] asserts that if $V$ is a neighborhood of zero for any consistent locally convex topology on $X$, then its polar $V^{\circ}$ is a $\sigma\left(X^{\prime}, X\right)$ compact subset of $X^{\prime}$.

The next theorem presents a crucial relationship between properness and supporting prices.

Theorem 22 Let $\left\langle E, E^{\prime}\right\rangle$ be a lattice pair and let $\tau$ be a consistent locally convex topology on $E$. Assume that $\succcurlyeq$ is a convex, monotone, preference relation on $E^{+}$that is also $(v, V)$ uniformly proper for some symmetric $\tau$-neighborhood $V$. Also, define the supporting price correspondence $\psi: E^{+} \rightarrow E^{\prime}$ by

$$
\psi(x)=\left\{p \in V^{\circ} \cap E_{+}^{\prime}: p \text { supports } \succcurlyeq \text { at } x \text { and } p \cdot v=1\right\} .
$$

Then we have the following.

1. The correspondence $\psi$ has nonempty, convex, and $\sigma\left(E^{\prime}, E\right)$-compact values.

2. If, in addition, the preference $\succcurlyeq$ is $\tau$-upper semicontinuous, then the correspondence $\psi$ is also $\left(\tau, w^{*}\right)$-upper hemicontinuous.

Proof: (1) Clearly, the correspondence $\psi$ has convex values. We first show that $\psi$ has nonempty values. Pick $x$ in $E^{+}$and set $P=\left\{y \in E^{+}: y \succcurlyeq x\right\}$ and $C=\bigcup_{\lambda>0} \lambda(-v+V)$. Clearly $P$ is a nonempty convex set and $C$ is a convex cone with the origin deleted having nonempty interior. Moreover $C \cap(P-x)=\varnothing$. Indeed if $\lambda(-v+w)=y-x$ with $\lambda>0$, $w \in V$, and $y \succcurlyeq x$, then $x-\lambda v+\lambda w=y \succcurlyeq x$, so by the properness of $\succcurlyeq$ we get $\lambda w \notin \lambda V$, or $w \notin V$, a contradiction. Now by the Separating Hyperplane Theorem [2, Theorem 4.46, p. 143], there is a nonzero $p \in E^{\prime}$ satisfying $p \cdot z \geqslant p \cdot y$ for all $z \in P-x$ and all $y \in C$. Since $C$ is a cone, it easily follows that $p \cdot z \geqslant 0 \geqslant p \cdot y$ for all $z \in P-x$ and all $y \in C$. In particular, we have $p \cdot y \geqslant p \cdot x$ for all $y \succcurlyeq x$. That is, $p$ supports $\succcurlyeq$ at $x$.

Next, note that $p \geqslant 0$. For if $z \geqslant 0$, then $x+z \geqslant x$, so $p \cdot(x+z) \geqslant p \cdot x$, which implies $p \cdot z \geqslant 0$. Next we show that $p \cdot v>0$. First note that $-v \in C$, so $p \cdot v \geqslant 0$. If $p \cdot v=0$, then $-v+V \subset C$ implies that $p \cdot u \leqslant 0$ for each $u \in V$. Since $V$ is symmetric, $p \cdot u=0$ for each $u \in V$, which in turn implies $p=0$, a contradiction. Replacing $p$ by $\frac{p}{p \cdot v}$, we may assume that $p \cdot v=1$. Now note that if $u \in V$, then $-v \pm u \in C$, so $p \cdot(-v \pm u) \leqslant 0$. Therefore, $\pm p \cdot u \leqslant p \cdot v=1$, or $|p \cdot u| \leqslant 1$ for each $u \in V$. That is, $p \in V^{\circ}$. Thus $p \in \psi(x)$, so $\psi$ has nonempty values. 
To see that the convex set $\psi(x)$ is $\sigma\left(E^{\prime}, E\right)$-compact note first that $\psi(x) \subset V^{\circ}$ and that $V^{\circ}$ is (by Alaoglu's Theorem) $\sigma\left(E^{\prime}, E\right)$-compact. It is easy to see that $\psi(x)$ is $\sigma\left(E^{\prime}, E\right)$-closed, so $\sigma\left(E^{\prime}, E\right)$-compact.

(2) To establish that $\psi$ is $\left(\tau, \sigma\left(E^{\prime}, E\right)\right)$-upper hemicontinuous, it suffices (since the range of $\psi$ is contained in the $\sigma\left(E^{\prime}, E\right)$-compact set $V^{\circ}$ ) to show that $\psi$ has a closed graph. To this end, assume $x_{\alpha} \stackrel{\tau}{\rightarrow} x, p_{\alpha} \in \psi\left(x_{\alpha}\right)$, and $p_{\alpha} \stackrel{\sigma\left(E^{\prime}, E\right)}{\longrightarrow} p$. We need to show that $z \succcurlyeq x$ implies $p \cdot z \geqslant p \cdot x$. So suppose that $z \succcurlyeq x$. Since $v$ is (by Lemma 17) desirable, for each $\varepsilon>0, z_{\varepsilon}=z+\varepsilon v$ satisfies $z_{\varepsilon} \succ x$. Since $x_{\alpha} \stackrel{\tau}{\rightarrow} x$ and $\succcurlyeq$ is $\tau$-upper semicontinuous, eventually $z_{\varepsilon} \succ x_{\alpha}$, and consequently $p_{\alpha} \cdot z_{\varepsilon} \geqslant p_{\alpha} \cdot x_{\alpha}$ eventually. Since $V^{\circ}$ is $\sigma\left(E^{\prime}, E\right)$-compact, the evaluation is jointly $\left(\tau, \sigma\left(E^{\prime}, E\right)\right)$-continuous on $E \times V^{\circ}$, so letting $\varepsilon \rightarrow 0$, in the limit $p \cdot z \geqslant p \cdot x$.

In essence, the notion of properness replaces the interiority assumption of the positive cone. Since only subspaces of $C(\Omega)$-spaces can have a positive cone with nonempty interior (see [2, Section 7.5]), the notion of properness provides the means of studying general equilibrium problems in infinite dimensional Riesz spaces whose cones have empty interiors. On the other hand, properness is not a panacea. The positive cone of an arbitrary Riesz space need not admit proper preferences that satisfy the other standard properties. For instance, for the Riesz space $\mathbb{R}_{\infty}$ (the Riesz space of real sequences equipped with the product topology) there is no proper continuous preference on $\mathbb{R}_{\infty}^{+}$ that can be represented by a quasiconcave and strictly monotone utility function; see $[8$, Example 3.6.9, p. 174].

As pointed out at the beginning of the section, the notion of properness has been used and studied extensively in the literature; see, for instance, C. D. Aliprantis, D. J. Brown, and O. Burkinshaw [5, 6, 7], A. Araujo and P. Monteiro [23], M. Florenzano [99], L. E. Jones [119, 121], M. A. Khan and N. T. Peck [126], A. Mas-Colell [152, 153], A. MasColell and S. F. Richard [154], A. Mas-Colell and W. R. Zame [155], S. F. Richard [184], S. F. Richard and S. Srivastava [185], S. F. Richard and W. R. Zame [186], N. C. Yannelis and W. R. Zame [220], and W. R. Zame [222].

\section{Properness and efficiency}

When preferences are uniformly proper the pathology of Example 15 cannot occur. A. Mas-Colell has established that under the additional assumption of uniform properness (and a few.other standard assumptions) the second welfare theorem is valid for Riesz exchange economies.

Theorem 23 (The Mas-Colell Second Welfare Theorem [152]) In a Riesz exchange economy, if preferences are convex, monotone, and uniformly $\tau$-proper for a consistent locally convex-solid topology $\tau$ on $E$, then every weakly Pareto efficient allocation is supported by prices. Moreover, if for each $i$ we pick a convex solid $\tau$-neighborhood $V_{i}$ of zero 
and $a$ vector $v_{i}>0$ that satisfy the definition of uniform properness for $\succcurlyeq_{i}$, then every weakly Pareto efficient allocation can be supported by a price $p>0$ that satisfies

$$
p \cdot\left(\sum_{i=1}^{m} v_{i}\right)=1 \text { and }|p \cdot z| \leqslant 1 \text { for all } z \in V=\bigcap_{i=1}^{m} V_{i} .
$$

For a proof see [152] or [8, Theorem 3.5.4, p. 154]. For lattice exchange economies A. MasColell and S. F. Richard established the following remarkable version of the second welfare theorem.

Theorem 24 (The Mas-Colell-Richard Second Welfare Theorem [154]) In a lattice exchange economy, if the preference of each consumer is convex, monotone, and $\omega$-uniformly proper, then every weakly efficient allocation can be supported by prices.

The proof of this theorem utilizes the lattice properties of the price space in an elegant manner. We indicate below the basic ideas involved. For details see [154].

We first set up some notation. We let $\mathbf{E}=E^{m}, \mathbf{E}^{\prime}=\left(E^{\prime}\right)^{m}$, and consider the lattice pair $\left\langle\mathbf{E}, \mathbf{E}^{\prime}\right\rangle$ under the obvious duality. The elements of $\mathbf{E}$ and $\mathbf{E}^{\prime}$ are indicated by boldface letters. The preference relation $\succcurlyeq$ on $\mathbf{E}^{+}$is defined by $\mathbf{x} \succcurlyeq \mathbf{y}$ if $x_{i} \succcurlyeq_{i} y_{i}$ for each $i$. As usual, a price $\mathbf{p}=\left(p_{1}, \ldots, p_{m}\right) \in \mathbf{E}^{\prime}$ supports $\mathbf{x} \in \mathbf{E}^{+}$if $\mathbf{y} \succcurlyeq \mathbf{x}$ implies $\mathbf{p} \cdot \mathbf{y} \geqslant \mathbf{p} \cdot \mathbf{x}$. (This is equivalent to the condition that if $x \succcurlyeq_{i} x_{i}$ for some $i$, then $p_{i} \cdot x \geqslant p_{i} \cdot x_{i}$.)

Lemma 25 (Mas-Colell-Richard) Consider a lattice exchange economy such that the preference of each consumer is convex, monotone, and $\omega$-uniformly proper. Then there exists a nonempty convex and weakly compact subset $\mathcal{K}$ of $\mathbf{E}_{+}^{\prime}$ consisting of nonzero prices with the following property: If $\mathbf{x} \in \mathbf{E}^{+}$is a weakly Pareto efficient allocation, then the subset $\mathcal{K}(\mathbf{x})$ of $\mathcal{K}$, defined by

$$
\mathcal{K}(\mathbf{x})=\{\mathbf{p} \in \mathcal{K}: \mathbf{p} \text { supports } \mathbf{x} \text { and } \mathbf{x} \text { maximizes } \mathbf{p} \text { over all allocations } \mathcal{A}\}
$$

is nonempty, convex, and weakly compact.

Proof of Theorem 24: Let $\mathrm{x}=\left(x_{1}, \ldots, x_{m}\right)$ be a weakly Pareto efficient allocation. Fix some $\mathbf{p}=\left(p_{1}, \ldots, p_{m}\right) \in \mathcal{K}(\mathbf{x})$ and let $\pi=\bigvee_{i=1}^{m} p_{i}>0$. From the definition of the members of $\mathcal{K}(\mathbf{x})$ and the definition of the supremum of a finite set of linear functionals, it follows that $\pi \cdot \omega=\sum_{i=1}^{m} p_{i} \cdot x_{i}$. Moreover, $\pi \geqslant p_{i}$ implies $\pi \cdot x_{i} \geqslant p_{i} \cdot x_{i}$ for each $i$. Summing up, we get $\pi \cdot \omega=\sum_{i=1}^{m} \pi \cdot x_{i} \geqslant \sum_{i=1}^{m} p_{i} \cdot x_{i}=\pi \cdot \omega$; so $\pi \cdot x_{i}=p_{i} \cdot x_{i}$ follows for each $i$.

Now, we claim that $\pi$ supports $\mathbf{x}$. To see this, let $x \succcurlyeq_{i} x_{i}$. Then $\pi \cdot x \geqslant p_{i} \cdot x \geqslant$ $p_{i} \cdot x_{i}=\pi \cdot x_{i}$, as desired.

For an illustration of Theorem 24 see the discussion after Example 59. 


\section{Walrasian Equilibrium}

In an exchange economy, the budget set of consumer $i$ at prices $p \in X^{\prime}$ is the set

$$
B_{i}(p)=\left\{x \in X_{i}: p \cdot x \leqslant p \cdot \omega_{i}\right\} .
$$

At prices $p$ consumer $i$ chooses a bundle from $B_{i}(p)$ that is best under the preference relation $\succcurlyeq_{i}$. Any such choice bundle is known as a demand vector for consumer $i$. When at some prices $p$ we can select demand bundles for all consumers that clear the market, then we say that the economy is in equilibrium. Formally, we have the following definition.

Definition 26 An allocation $\left(x_{1}, \ldots, x_{m}\right)$ in an exchange economy is:

- a competitive (or a Walrasian) equilibrium if for some nonzero price $p \in X^{\prime}$, each $x_{i}$ is a maximal element in consumer $i$ 's budget set $B_{i}(p)$.

- $a$ quasiequilibrium if there exists some nonzero price $p \in X^{\prime}$ such that $x \succcurlyeq_{i} x_{i}$ in $X_{i}$ implies $p \cdot x \geqslant p \cdot \omega_{i}$.

For simplicity, a competitive equilibrium is also called an equilibrium. Here are some elementary properties of equilibria.

Lemma 27 For an allocation $\left(x_{1}, \ldots, x_{m}\right)$ in an exchange economy we have:

1. The allocation $\left(x_{1}, \ldots, x_{m}\right)$ is a competitive equilibrium with respect to some nonzero price $p \in X^{\prime}$ if and only if $x_{i} \in B_{i}(p)$ for each $i$ and $x \succ_{i} x_{i}$ in $X_{i}$ implies $p \cdot x>p \cdot \omega_{i}$.

2. If each consumer has a desirable bundle, then every competitive equilibrium is a quasiequilibrium.

Proof: The proof of (1) is well known.

(2) Assume that $\left(x_{1}, \ldots, x_{m}\right)$ is a competitive equilibrium with respect to some nonzero price $p \in X^{\prime}$ and let $v_{i}$ be a desirable commodity for consumer $i$. So if $x \succcurlyeq_{i} x_{i}$ in $X_{i}$, then there exists some $\delta>0$ such that $0<\alpha<\delta$ implies $x+\alpha v_{i} \succ_{i} x_{i}$, and consequently

$$
p \cdot\left(x+\alpha v_{i}\right)=p \cdot x+\alpha p \cdot v_{i}>p \cdot \omega_{i}
$$

for each $0<\alpha<\delta$. Letting $\alpha \rightarrow 0^{+}$, we get $p \cdot x \geqslant p \cdot \omega_{i}$, so that $\left(x_{1}, \ldots, x_{m}\right)$ is a quasiequilibrium.

Theorem 30 below gives conditions under which a quasiequilibrium is also an equilibrium. The next set of results includes a few more properties of equilibria.

Lemma 28 If $\left(x_{1}, \ldots, x_{m}\right)$ is a quasiequilibrium in an exchange economy with respect to a nonzero price $p \in X^{\prime}$, then $p \cdot x_{i}=p \cdot \omega_{i}$ for each $i$ (so the price $p$ supports the allocation). 
Because of the preceding result, any nonzero price that makes an allocation an equilibrium or a quasiequilibrium is referred to as a supporting price for the allocation.

Lemma 29 In a Riesz commodity exchange economy, if one consumer has a strictly monotone preference, then every price supporting an equilibrium is strictly positive.

Proof: Assume that the first consumer has a strictly monotone preference and that a non-zero price $p$ supports an equilibrium $\left(x_{1}, \ldots, x_{m}\right)$. By Lemma 12 , we know that the price $p$ is positive. Now let $x>0$. Then the strict monotonicity of $\succcurlyeq_{1}$ implies $x_{1}+x \succ_{1} x_{1}$. This implies $p \cdot\left(x_{1}+x\right)=p \cdot x_{1}+p \cdot x>p \cdot \omega_{1}=p \cdot x_{1}$, so $p \cdot x>0$, proving that $p \gg 0$.

In a quasiequilibrium, if consumers have positive income, then in fact it is an equilibrium.

Theorem 30 Assume that in a lattice exchange economy preferences are continuous for some linear topology. If an allocation $\left(x_{1}, \ldots, x_{m}\right)$ is a quasiequilibrium with respect to a nonzero price $p \in E^{\prime}$ and $p \cdot \omega_{i}>0$ for some $i$, then $x_{i}$ is a maximal element in $B_{i}(p)$. In particular, if $p \cdot \omega_{i}>0$ for each $i$, then the allocation $\left(x_{1}, \ldots, x_{m}\right)$ is a competitive equilibrium.

Proof: By Lemma 28, we know that $p \cdot x_{i}=p \cdot \omega_{i}$, so $x_{i} \in B_{i}(p)$ for each $i$. Now let $p \cdot \omega_{i}>0$ for some $i$, and assume by way of contradiction that there exists some $x \in B_{i}(p)$ (i.e., $p \cdot x \leqslant p \cdot \omega_{i}$ ) such that $x \succ_{i} x_{i}$. Since the preference $\succcurlyeq_{i}$ is continuous for some linear topology, there exists some $0<\delta<1$ such that $\delta x \succ_{i} x_{i}$. Since $p$ supports the allocation, we see that

$$
\delta p \cdot x=p \cdot(\delta x) \geqslant p \cdot \omega_{i} .
$$

On the other hand, we have $\delta p \cdot x \leqslant \delta p \cdot \omega_{i}<p \cdot \omega_{i}$, which is impossible, and the conclusion follows.

Corollary 31 For a lattice exchange economy assume that for some linear topology every preference relation is continuous. If a quasiequilibrium is supported by a price $p$ with $p \cdot \omega>0$, then the quasiequilibrium is weakly Pareto efficient.

Proof: Repeat the proof of Theorem 13 and use Theorem 30.

Corollary 32 In a lattice exchange economy, if preferences are strictly monotone and continuous for some linear topology and the total endowment is strictly positive, then an allocation is a competitive equilibrium if and only if it is a quasiequilibrium. Moreover, in this case, every nonzero price that supports a quasiequilibrium must be strictly positive.

Proof: Let $\left(x_{1}, \ldots, x_{m}\right)$ be a quasiequilibrium supported by a nonzero price $p$. Clearly, $p>0$. We claim that $p \gg 0$ is also true. To see this, start by noticing that $\omega \gg 0$ implies $p \cdot \omega=\sum_{i=1}^{m} p \cdot \omega_{i}>0$. So $p \cdot \omega_{i}>0$ for some $i$. By Theorem 30 the bundle $x_{i}$ 
is a maximal element in $B_{i}(p)$. Now let $x>0$. If $p \cdot x=0$, then $p \cdot\left(x_{i}+x\right)=p \cdot \omega_{i}$ (i.e., $\left.x+x_{i} \in B_{i}(p)\right)$ and by the strict monotonicity of $\succcurlyeq_{i}$, we see that $x+x_{i} \succ_{i} x_{i}$, which contradicts the maximality of $x_{i}$. Thus, $p \cdot x>0$, which means that $p$ is strictly positive. Now to complete the proof notice that $p \cdot \omega_{i}>0$ for each $i$ and then invoke Theorem 30 .

In general, a quasiequilibrium need not be a competitive equilibrium. Here is a simple example.

Example 33 Consider a two consumer 2-dimensional exchange economy with the following characteristics:

- Consumer 1: Initial endowment $\omega_{1}=\left(\frac{1}{2}, 0\right)$ and preference $\succcurlyeq_{1}$ represented by the utility function $u_{1}(x, y)=x+y$.

- Consumer 2: Initial endowment $\omega_{2}=\left(\frac{1}{2}, 1\right)$ and preference $\succcurlyeq_{2}$ represented by the utility function $u_{2}(x, y)=y$.

A straightforward argument shows that the allocation $((1,0),(0,1))$ is a quasiequilibrium with respect to the price $p=(0,1)$ but fails to be a competitive equilibrium.

Since $(1,0) \succ_{1} \omega_{1}$ and $(0,1) \succcurlyeq_{2} \omega_{2}$, observe that $\left(\omega_{1}, \omega_{2}\right)$ is not a Pareto efficient allocation. However, it is easy to see that $\left(\omega_{1}, \omega_{2}\right)$ is weakly Pareto efficient.

\section{Continuity properties of supporting prices}

Consider an allocation $\left(x_{1}, \ldots, x_{m}\right)$ in an exchange economy. We say that a linear functional $p$ (called a price) on a vector subspace $Y$ of the commodity space that contains each $x_{i}$ supports the allocation $\left(x_{1}, \ldots, x_{m}\right)$ on $Y$ if $x \in X_{i} \cap Y$ and $x \succcurlyeq_{i} x_{i}$ imply $p \cdot x \geqslant p \cdot x_{i}$. Our purpose here is to discuss two important continuity properties of supporting prices.

Recall that if $E$ is a Riesz space and $z \in E^{+}$, then the principal ideal (or simply the ideal) $E_{z}$ generated by $z$ is the vector subspace

$$
E_{z}=\{x \in E: \exists \lambda>0 \text { such that }|x| \leqslant \lambda z\} .
$$

If we define the real function $\|\cdot\|_{\infty}: E_{z} \rightarrow[0, \infty)$ by

$$
\|x\|_{\infty}=\inf \{\lambda>0:|x| \leqslant \lambda z\}
$$

then $\|\cdot\|_{\infty}$ is a lattice seminorm on $E_{z}$ (i.e., $|x| \leqslant|y|$ implies $\|x\|_{\infty} \leqslant\|y\|_{\infty}$ ). If $E$ is an Archimedean Riesz space, then $\|\cdot\|_{\infty}$ is also a norm, and in fact an M-norm. In case $E$ is an Archimedean uniformly complete Riesz space (in particular a Banach lattice), then $E_{z}$ is an AM-space-in which case $\left(E_{z},\|\cdot\|_{\infty}\right)$ is lattice isometric to a $C(\Omega)$-space ( $\Omega$ 
Hausdorff and compact) with $z$ corresponding to the constant function one on $\Omega$. The norm dual $E_{z}^{\prime}$ of $\left(E_{z},\|\cdot\|_{\infty}\right)$ is an AL-space-which is lattice isometric to some concrete $L_{1}(\mu)$-space. For details on AM- and AL-spaces see [11] and [2, Section 7.4].

Returning now to our setting, we see that the Edgeworth box $[0, \omega]$ incorporates all the economic activities of a Riesz commodity exchange economy. In fact, $[0, \omega]$ lies entirely in $E_{\omega}$. Therefore, although the commodity-price duality of the economy is described in terms of the dual pair $\left\langle E, X^{\prime}\right\rangle$, the underlying Riesz pair $\left\langle E_{\omega}, E_{\omega}^{\prime}\right\rangle$ is a more realistic Riesz pair that describes the commodity-price duality of the economy. This Riesz pair appears over and over in the following discussion.

Theorem 34 (Aliprantis-Brown-Burkinshaw [5]) In a Riesz exchange economy assume:

a. Preferences are lower semicontinuous for a consistent locally convex-solid topology $\tau$ on $E$.

b. Every consumer desires the total endowment $\omega$.

Assume further that a positive linear functional $p$ on $E_{\omega}$ (the ideal generated by $\omega$ in $E$ ) supports an allocation. Then:

1. The linear functional $p$ is $\tau$-continuous on the Edgeworth box $[0, \omega]$.

2. If, in addition, $[0, \omega]$ is weakly compact, then $p$ is also order continuous on $E_{\omega}$, i.e., $z_{\alpha} \downarrow 0$ in $E_{\omega}$ implies $p \cdot z_{\alpha} \downarrow 0$.

Proof: Let a positive price $p$ on $E_{\omega}$ support the allocation $\left(x_{1}, \ldots, x_{m}\right)$.

(1) Consider first the case of a net $\left\{v_{\alpha}\right\}$ that satisfies $0 \leqslant v_{\alpha} \leqslant x_{i}$ for some $i$ and all $\alpha$ and $v_{\alpha} \stackrel{\tau}{\rightarrow} 0$. Fix $\varepsilon>0$. From $x_{i}-v_{\alpha}+\varepsilon \omega \stackrel{\tau}{\alpha} x_{i}+\varepsilon \omega, x_{i}+\varepsilon \omega \succ_{i} x_{i}$, and the lower $\tau$-semicontinuity of $\succcurlyeq_{i}$, we see that there exists some $\alpha_{0}$ such that $x_{i}-v_{\alpha}+\varepsilon \omega \succ_{i} x_{i}$ for all $\alpha \geqslant \alpha_{0}$. So

$$
p \cdot\left(x_{i}-v_{\alpha}+\varepsilon \omega\right)=p \cdot x_{i}-p \cdot v_{\alpha}+\varepsilon p \cdot \omega \geqslant p \cdot x_{i}
$$

for all $\alpha \geqslant \alpha_{0}$. Thus, $0 \leqslant p \cdot v_{\alpha} \leqslant \varepsilon p \cdot \omega$ for all $\alpha \geqslant \alpha_{0}$, which means that $\lim _{\alpha} p \cdot v_{\alpha}=0$.

Next, we consider a net $\left\{v_{\alpha}\right\}$ satisfying $0 \leqslant v_{\alpha} \leqslant \omega=\sum_{i=1}^{m} x_{i}$ for each $\alpha$ and $v_{\alpha} \stackrel{\tau}{\rightarrow} 0$. By the Riesz Decomposition Property [2, Section 6.5, p. 215], we can write $v_{\alpha}=\sum_{i=1}^{m} v_{\alpha}^{i}$ with $0 \leqslant v_{\alpha}^{i} \leqslant x_{i}$. Since $0 \leqslant v_{\alpha}^{i} \leqslant v_{\alpha}$ for all $\alpha$ and all $i$, it follows from the local solidness of $\tau$ that $v_{\alpha}^{i} \underset{\alpha}{\stackrel{\tau}{\alpha}} 0$. By the preceding case, we get

$$
\lim _{\alpha} p \cdot v_{\alpha}=\lim _{\alpha} \sum_{i=1}^{m} p \cdot v_{\alpha}^{i}=\sum_{i=1}^{m} \lim _{\alpha} p \cdot v_{\alpha}^{i}=0 .
$$


Finally, we consider the general case. That is, assume that a net $\left\{v_{\alpha}\right\}$ in $[0, \omega]$ satisfies $v_{\alpha} \stackrel{\tau}{\rightarrow} v$. Then $\left|v_{\alpha}-v\right| \leqslant \omega$ for each $\alpha$ and $\left|v_{\alpha}-v\right| \stackrel{\tau}{\rightarrow} 0$. Now use the preceding case and the inequality $\left|p \cdot v_{\alpha}-p \cdot v\right| \leqslant p \cdot\left|v_{\alpha}-v\right|$ to conclude that $\lim _{\alpha} p \cdot v_{\alpha}=p \cdot v$.

(2) Assume $\left\{v_{\alpha}\right\} \subseteq[0, \omega]$ for each $\alpha$ and $v_{\alpha} \downarrow 0$. Since $[0, \omega]$ is weakly compact, it follows that $\left\{v_{\alpha}\right\}$ has a weakly convergent subnet and from this, we infer that $v_{\alpha} \stackrel{w}{\longrightarrow} 0$.

Now let $V$ be a convex, solid and $\tau$-closed neighborhood of zero. Notice that $E_{+}^{\prime} \cap V^{\circ}$ (where $V^{\circ}$ is the polar of $V$ in $E^{\prime}$ ) equipped with the $w^{*}$-topology is a compact set (see [2, Theorem 4.79]). In addition, if we consider each $v_{\alpha}$ as a real valued function on $E_{+}^{\prime} \cap V^{\mathrm{O}}$ via the formula $v_{\alpha}(f)=f\left(v_{\alpha}\right)$, then each $v_{\alpha}$ is continuous. From $v_{\alpha} \stackrel{w}{\longrightarrow} 0$, we see that $v_{\alpha}(f) \downarrow 0$ for each $f \in E_{+}^{\prime} \cap V^{\circ}$. Thus, from Dini's Theorem [2, Theorem 2.62], the net $\left\{v_{\alpha}\right\}$ converges uniformly to zero on $E_{+}^{\prime} \cap V^{\circ}$. An easy argument now shows that there exists some $\alpha_{0}$ such that $v_{a} \in V^{\circ \circ}=V$ for all $\alpha \geqslant \alpha_{0}$. Hence, $v_{\alpha} \stackrel{\tau}{\rightarrow} 0$, as desired.

A positive linear functional that supports an allocation on $E_{\omega}$ need not be $\tau$-continuous on $E_{\omega}$ (although it is $\tau$-continuous on the Edgeworth box $[0, \omega]$ ). An example follows.

Example 35 (Aliprantis-Burkinshaw [12]) Consider the Riesz exchange economy discussed in Example 15. Notice that

$$
\omega=\omega_{1}+\omega_{2}=\left(\frac{1}{2^{2}}, \frac{1}{2^{4}}, \ldots, \frac{1}{2^{2 n}}, \ldots\right) .
$$

Clearly, $\omega \gg 0$, so $E_{\omega}$ is norm dense in $\ell_{1}$, i.e., $\overline{E_{\omega}}=\ell_{1}$.

Now consider the price $p=\left(2,2^{2}, 2^{3}, \ldots, 2^{n}, \ldots\right)$. Then $p$ defines a positive price on $E_{\omega}$; in fact, notice that $p \cdot \omega=\sum_{n=1}^{\infty} \frac{1}{2^{n}}=1$. We claim that $p$ supports the allocation $\left(\omega_{1}, \omega_{2}\right)$ on $E_{\omega}$ as a Walrasian equilibrium. This follows from the following two facts.

$$
\text { (1) } u\left(\omega_{i}\right)=p \cdot \omega_{i}=\frac{1}{2} \quad \text { and } \quad(2) p \cdot x \geqslant u(x) \text { for each } x \in \ell_{1}^{+} \cap E_{\omega} \text {. }
$$

To see (1), note that $\varphi_{n}\left(\frac{1}{2^{2 n+1}}\right)=2^{n} \cdot \frac{1}{2^{2 n+1}}=\frac{1}{2^{n+1}}$, so

$$
u\left(\omega_{i}\right)=\sum_{n=1}^{\infty} \varphi_{n}\left(\frac{1}{2^{2 n+1}}\right)=\sum_{n=1}^{\infty} \frac{1}{2^{n+1}}=p \cdot \omega_{i}=\frac{1}{2} .
$$

For (2) notice first $t \geqslant 0$ implies $2^{n} t \geqslant \varphi_{n}(t)$ for each $n$. Indeed, if $t \leqslant \frac{1}{2^{2 n}}$, then $\varphi_{n}(t)=2^{n} t$. On the other hand, if $t>\frac{1}{2^{2 n}}$, then

$$
2^{n} t=\left(2^{n}-1\right) t+t \geqslant\left(2^{n}-1\right) \cdot \frac{1}{2^{2 n}}+t=\frac{1}{2^{n}}-\frac{1}{2^{2 n}}+t=\varphi_{n}(t) .
$$

Consequently, for $x=\left(x_{1}, x_{2}, \ldots\right) \in \ell_{1}^{+} \cap E_{\omega}$, we have

$$
p \cdot x=\sum_{n=1}^{\infty} 2^{n} x_{n} \geqslant \sum_{n=1}^{\infty} \varphi_{n}\left(x_{n}\right)=u(x) .
$$


Now observe that if $x \succ_{i} \omega_{i}$ in $E_{\omega}^{+}$, then $p \cdot x \geqslant u(x)>u\left(\omega_{i}\right)=p \cdot \omega_{i}$, so $p \cdot x>p \cdot \omega_{i}$. In other words, $p$ supports $\left(\omega_{1}, \omega_{2}\right)$ on $E_{\omega}$ as a Walrasian equilibrium. Finally, note that the price $p$ is not $\ell_{1}$-norm continuous on $E_{\omega}$. Indeed, if $x_{n}=\left(0, \ldots, 0, \frac{1}{2^{n}}, 0,0, \ldots\right) \in E_{\omega}$, then $\left\|x_{n}\right\|_{1}=\frac{1}{2^{n}} \rightarrow 0$ while $p \cdot x_{n}=1$ for each $n$.

The pathology of Example 35 cannot occur in a Riesz exchange economy with proper preferences. N. C. Yannelis and W. R. Zame have shown that when preferences are proper, every price that supports an allocation on the ideal $E_{\omega}$ is necessarily topologically continuous on $E_{\omega}$.

Theorem 36 (Yannelis-Zame [220]) In a Riesz exchange economy, if every preference is uniformly $\tau$-proper on $E_{\omega}$ for some locally convex-solid consistent topology $\tau$ on $E$, then every positive price on $E_{\omega}$ that supports an allocation is $\tau$-continuous on $E_{\omega}$.

Proof: Consider a Riesz exchange economy satisfying the properties of the theorem and let an allocation $\left(x_{1}, \ldots, x_{m}\right)$ be supported by a price $0<p \in E_{\omega}^{\prime}$. Since every vector of $E_{\omega}^{+}$is bounded by a multiple of $\omega$, it follows that $\omega$ is a vector of uniform properness. So there exists a convex, solid $\tau$-neighborhood $V$ of zero such that $x-\alpha \omega+z \succcurlyeq_{i} x$ in $E_{\omega}^{+}$ and $\alpha>0$ imply $z \notin \alpha V$.

Next, consider the Minkowski lattice seminorm $\rho$ of $V$ defined on $E$ by the formula $\rho(y)=\inf \{\lambda>0: y \in \lambda V\}$ for each $y \in E$. Clearly, $\rho$ is a $\tau$-continuous seminorm on $E$ and, as we shall see, one of its multiples dominates $p$ on $E_{\omega}$.

Assume first $0 \leqslant z \leqslant \omega=\sum_{i=1}^{m} x_{i}$. Then by the Riesz Decomposition Property we can write $z=\sum_{i=1}^{m} z_{i}$ with $0 \leqslant z_{i} \leqslant x_{i}$ for each $i$. Put $\alpha_{i}=\rho\left(z_{i}\right)$ and let $\varepsilon>0$ be fixed. For each $i$ let $y_{i}=x_{i}+\left(\alpha_{i}+\varepsilon\right) \omega-z_{i} \geqslant 0$. Clearly, $x_{i}=y_{i}-\left(\alpha_{i}+\varepsilon\right) \omega+z_{i} \geqslant 0$. If $y_{i}-\left(\alpha_{i}+\varepsilon\right) \omega+z_{i} \succcurlyeq_{i} y_{i}$, then by the uniform $\tau$-properness of $\succcurlyeq_{i}$ on $E_{\omega}$, we see that $z_{i} \notin\left(\alpha_{i}+\varepsilon\right) V$, which contradicts $\rho\left(z_{i}\right)=\alpha_{i}$. Therefore, $y_{i} \succ_{i} y_{i}-\left(\alpha_{i}+\varepsilon\right) \omega+z_{i}=x_{i}$. Since $p$ supports $\left(x_{1}, \ldots, x_{m}\right)$ on $E_{\omega}$, it follows that

$$
p \cdot y_{i} \geqslant p \cdot x_{i}=p \cdot\left[y_{i}-\left(\alpha_{i}+\varepsilon\right) \omega+z_{i}\right]=p \cdot y_{i}-\left(\alpha_{i}+\varepsilon\right) p \cdot \omega+p \cdot z_{i} .
$$

Hence, $p \cdot z_{i} \leqslant\left(\alpha_{i}+\varepsilon\right) p \cdot \omega$ for each $i$ and all $\varepsilon>0$, from which it follows that $p \cdot z_{i} \leqslant$ $\alpha_{i} p \cdot \omega=(p \cdot \omega) \rho\left(z_{i}\right) \leqslant(p \cdot \omega) \rho(z)$. This implies

$$
p \cdot z=\sum_{i=1}^{m} p \cdot z_{i} \leqslant\left(\sum_{i=1}^{m} p \cdot \omega\right) \rho(z)=m(p \cdot \omega) \rho(z)
$$

for all $z$ with $0 \leqslant z \leqslant \omega$. Now consider any $z \in E_{\omega}$. Pick some $\lambda>0$ such that $|z| \leqslant \lambda \omega$ and note that

$$
|p \cdot z| \leqslant p \cdot|z|=\lambda p \cdot\left(\frac{1}{\lambda}|z|\right) \leqslant \lambda m(p \cdot \omega) \rho\left(\frac{1}{\lambda}|z|\right)=m(p \cdot \omega) \rho(z) .
$$

Thus, the price $p$ is dominated by a $\tau$-continuous seminorm on $E_{\omega}$ and therefore is itself $\tau$-continuous on $E_{\omega}$. 


\section{The utility space and efficiency}

In this section, we discuss the utility space of an exchange economy. We consider a Riesz commodity exchange economy with commodity-price dual pair $\left\langle E, X^{\prime}\right\rangle$ in which preferences are represented by monotone quasiconcave utility functions. The utility function of consumer $i$ is written $u_{i}$. Without loss of generality, we further assume that $u_{i}(0)=0$. Unless otherwise stated, when we say that a utility function is continuous it should be understood that the utility function is continuous for some consistent locally convex topology on $E$.

A free disposal allocation is an $m$-tuple $\left(x_{1}, \ldots, x_{m}\right)$ satisfying $x_{i} \in X_{i}$ for each $i$ and $\sum_{i=1}^{m} x_{i} \leqslant \omega$. A utility allocation is vector of the form $\left(u_{1}\left(x_{1}\right), \ldots, u_{m}\left(x_{m}\right)\right)$, where $\left(x_{1}, \ldots, x_{m}\right)$ is a free disposal allocation. If $x=\left(x_{1}, \ldots, x_{m}\right)$ is a free disposal allocation, then for simplicity we write $u(x)$ for $\left(u_{1}\left(x_{1}\right), \ldots, u_{m}\left(x_{m}\right)\right)$.

Definition 37 The set of utility allocations is called the utility space of the economy, denoted $\mathbf{U}$, i.e.,

$$
\mathbf{U}=\left\{u(x)=\left(u_{1}\left(x_{1}\right), \ldots, u_{m}\left(x_{m}\right)\right): x=\left(x_{1}, \ldots, x_{m}\right) \text { is a free disposal allocation }\right\} .
$$

The utility space enjoys two simple properties.

Lemma 38 For a Riesz commodity exchange economy we have the following.

1. The utility space $\mathbf{U}$ is comprehensive in the sense that if $0 \leqslant y \leqslant z$ in $\mathbb{R}^{m}$ and $z \in \mathbf{U}$, then $y \in \mathbf{U}$.

2. If $\omega$ is desirable for each consumer, then there exists some $\varepsilon>0$ such that $0 \leqslant z \in$ $\mathbb{R}^{m}$ with $\|z\|<\varepsilon$ implies $z \in \mathrm{U}$.

Proof: (1) To see this, let $0 \leqslant y=\left(y_{1}, \ldots, y_{m}\right) \leqslant z=\left(z_{1}, \ldots, z_{m}\right) \in \mathrm{U}$. Pick a free disposal allocation $\left(x_{1}, \ldots, x_{m}\right)$ with $z_{i}=u_{i}\left(x_{i}\right)$ for each $i$. Since the real valued function $f_{i}(t)=u_{i}\left(t x_{i}\right), 0 \leqslant t \leqslant 1$, is continuous, there exists (by the Intermediate Value Theorem) some $0 \leqslant t_{i} \leqslant 1$ such that $y_{i}=u_{i}\left(t_{i} x_{i}\right)$. Clearly, $\left(t_{1} x_{1}, \ldots, t_{m} x_{m}\right)$ is a free disposal allocation, so $y \in \mathrm{U}$.

(2) If $\omega$ is desirable for each consumer, then $u_{i}\left(\frac{1}{m} \omega\right)>0$ for each $i$. So by part (1), the number $\varepsilon=\min \left\{u_{i}\left(\frac{1}{m} \omega\right): i=1, \ldots, m\right\}$ satisfies the desired property.

Since (in the case of monotone preferences) for any free disposal allocation $\left(x_{1}, \ldots, x_{m}\right)$ we have $u_{i}\left(x_{i}\right) \leqslant u_{i}(\omega)$ for each $i$, we see that the utility space $\mathrm{U}$ is always a (nonempty) bounded subset of $\mathbb{R}_{+}^{m}$. When is $\mathbf{U}$ compact? Or, equivalently, When is the utility space $\mathrm{U}$ closed? The next theorem provides the answer.

Lemma 39 In a Riesz commodity exchange economy whose consumer preferences are represented by monotone continuous utility functions, the following statements are equivalent. 
1. The utility space $\mathbf{U}$ is a closed subset of $\mathbb{R}_{+}^{m}$.

2. The utility space $\mathbf{U}$ is a compact subset of $\mathbb{R}_{+}^{m}$.

3. If a sequence of free disposal allocations $\left\{\left(x_{1}^{n}, \ldots, x_{m}^{n}\right)\right\}$ satisfies $x_{i}^{n+1} \succcurlyeq_{i} x_{i}^{n}$ for all $i$ and all $n$, then there is an allocation $\left(x_{1}, \ldots, x_{m}\right)$ such that $x_{i} \succcurlyeq_{i} x_{i}^{n}$ for all $i$ and all $n$.

Definition 40 An exchange economy is utility closed if its utility space is a closed set (or equivalently, if its utility space $\mathrm{U}$ satisfies any one of the equivalent statements of Lemma 39).

We emphasize once more that for an exchange economy to have a utility space it is necessary that consumer preferences be represented by utility functions.

Lemma 41 In a Riesz commodity exchange economy, if

1. the Edgeworth box $[0, \omega]$ is weakly compact, and

2. consumer preferences are represented by monotone continuous utility functions,

then the economy is utility closed.

An economy might be utility closed even without the Edgeworth box $[0, \omega]$ being weakly compact. Here is an example.

Example 42 Consider a two consumer Riesz exchange economy with the following characteristics.

- Riesz pair: $\langle C[0,1], c a[0,1]\rangle$.

- Total endowment: $\omega=\mathbf{1}$ (the constant function one).

- Consumer 1: Utility function $u_{1}(x)=\int_{0}^{1} x(t) d t$.

- Consumer 2: Utility function $u_{2}(x)=\int_{0}^{1} \sqrt{x(t)} d t$.

You can verify that:

1. Each utility function is (sup) norm eontinuous; concave, and strictly monotone;

2. The Edgeworth box $[0, \omega]$ is not weakly compact; and

3. The utility space of the economy is given by

$$
\mathbf{U}=\left\{(x, y) \in \mathbb{R}_{+}^{2}: x+y^{2} \leqslant 1\right\} .
$$


In particular, the economy is utility closed.

The weakly Pareto efficient allocations are closely related with the boundary points of the utility space. The next result clarifies the relationship.

Lemma 43 In a Riesz commodity exchange economy whose consumer preferences are represented by monotone continuous utility functions we have the following.

1. If $x=\left(x_{1}, \ldots, x_{m}\right)$ is a weakly Pareto efficient allocation, then its utility allocation vector $u(x)=\left(u_{1}\left(x_{1}\right), \ldots, u_{m}\left(x_{m}\right)\right)$ is a boundary point of the utility space relative to $\mathbb{R}_{+}^{m}$ (i.e., a boundary point of $\mathrm{U}$ considered as a subset of $\mathbb{R}_{+}^{m}$ ).

2. If $z \in \mathrm{U}$ is a boundary point of the utility space relative to $\mathbb{R}_{+}^{m}$, then there exists a weakly Pareto efficient allocation $\left(x_{1}, \ldots, x_{m}\right)$ such that $z=\left(u_{1}\left(x_{1}\right), \ldots, u_{m}\left(x_{m}\right)\right)$.

We now introduce the function $\rho: \Delta \rightarrow[0, \infty)$ via the formula

$$
\rho(s)=\sup \{\alpha \geqslant 0: \alpha s \in \mathbf{U}\},
$$

where as usual $\Delta=\left\{s \in \mathbb{R}_{+}^{m}: \sum_{i=1}^{m} s_{i}=1\right\}$.

Lemma 44 For a Riesz commodity exchange economy, assume preferences are representable by monotone and continuous utility functions and that the total endowment is desirable for each consumer. Then the function $\rho: \Delta \rightarrow[0, \infty)$, defined by

$$
\rho(s)=\sup \{\alpha \geqslant 0: \alpha s \in \mathrm{U}\},
$$

is continuous.

This follows from the Berge Maximum Theorem, see [8, Theorem 3.5.7, p. 157].

We can define another function $\mathbf{b}: \Delta \rightarrow \partial \mathbf{U}$ via the formula $\mathbf{b}(s)=\rho(s) s$. Note that indeed $\mathbf{b}(s) \in \partial \mathbf{U}$ for each $s \in \Delta$. The function $\mathbf{b}$ is called the boundary (utility) function of the economy. From Lemma 44 we see that $\mathbf{b}$ is also a continuous function.

Definition 45 The correspondence $\mathcal{B}: \Delta \rightarrow \mathcal{A}$, defined by

$$
\mathcal{B}(s)=\{x \in \mathcal{A}: u(x) \geqslant \mathbf{b}(s)\},
$$

is called the boundary (utility) allocation correspondence.

For utility closed Riesz commodity exchange economies whose preferences are represented by monotone continuous utility functions, Lemma 43 shows that for each $s \in \Delta$ there is an allocation $x \in \mathcal{A}_{w p}$ such that $u(x)=\rho(s) s$. Thus, in this case, the boundary allocation correspondence in nonempty-valued. Actually, much more is true. 
Lemma 46 For a Riesz commodity exchange economy, assume preferences are represented by monotone quasiconcave and continuous utility functions, the total endowment is desired by each consumer, and that the Edgeworth box $[0, \omega]$ is weakly compact. Then the utility allocation correspondence $\mathcal{B}: \Delta \rightarrow \mathcal{A}_{\text {wp }}$, defined by

$$
\mathcal{B}(s)=\{x \in \mathcal{A}: u(x) \geqslant \mathbf{b}(s)\},
$$

has nonempty, convex, and compact values and is continuous (i.e., upper and lower hemicontinuous).

Proof: The proof is straightforward. Note that since $\mathcal{A}_{w p}$ is Hausdorff and weakly compact (Theorem 7), the upper hemicontinuity of $\mathcal{B}$ is equivalent to $\mathcal{B}$ having closed graph $[2$, Theorem 14.12, p. 465].

\section{Existence of equilibria}

Corollary 31 asserts that in general, if quasiequilibria exist, then they are weakly Pareto efficient. Under appropriate hypotheses, A. Mas-Colell has shown that there do indeed exist quasiequilibria.

Theorem 47 (Mas-Colell [152]) Assume that a Riesz exchange economy satisfies the following properties.

1. The preferences of each consumer are represented by a monotone and quasiconcave utility function.

2. There is a consistent locally convex-solid topology $\tau$ on the commodity space such that each utility function is continuous.

3. Each preference is uniformly $\tau$-proper.

4. The total endowment is desirable for each consumer.

5. The exchange economy is utility closed.

Then the exchange economy has a quasiequilibrium.

For a proof see $[8$, Theorem 3.5 .12 , p. 161$]$.

The next important result regarding lattice exchange economies is due to A. MasColell and S. F. Richard [154].

Theorem 48 (Mas-Colell-Richard [154]) Assume that in a lattice exchange economy:

1. Its Edgeworth box $[0, \omega]$ is weakly compact; and 
2. The preference of each consumer is w-uniformly proper and is represented by a monotone, quasiconcave, and continuous utility function.

Then the economy has a quasiequilibrium.

Proof: We sketch the basic steps of the Mas-Colell-Richard proof. See [154] for the details. For each $\mathbf{p}=\left(p_{1}, \ldots, p_{m}\right) \in \mathbf{E}^{\prime}$, let $\pi_{\mathbf{p}}$ denote the supremum of the set $\left\{p_{1}, \ldots, p_{m}\right\}$, i.e., $\pi_{\mathbf{p}}=\bigvee_{i=1}^{m} p_{i} \in E^{\prime}$. Next we use the nonempty, convex, and weakly compact set $\mathcal{K}$ guaranteed by Lemma 25 .

Introduce the correspondence $\Phi: \Delta \nrightarrow T=\left\{\left(t_{1}, \ldots, t_{m}\right) \in \mathbb{R}^{m}: \sum_{i=1}^{m} t_{i}=0\right\}$ defined via the formula

$\Phi(s)=\left\{\left(t_{1}, \ldots, t_{m}\right) \in T: \exists \mathbf{x} \in \mathcal{B}(s)\right.$ and $\mathbf{p} \in \mathcal{K}(\mathbf{x})$ such that $t_{i}=\pi_{\mathbf{p}}\left(\omega_{i}-x_{i}\right)$ for each $\left.i\right\}$ where $\mathcal{B}$ is the correspondence introduced in Definition 45 . Since $\pi_{\mathrm{p}}$ supports $\mathbf{x}$, it suffices to show that $0 \in \Phi(s)$ for some $s \in \Delta$.

This is established by proving that the correspondence $\Phi$ is nonempty, convex, and compact valued and also upper hemicontinuous. It then follows that the correspondence $\Psi: \Delta \nrightarrow \mathbb{R}^{m}$ defined by $\Psi(s)=s+\Phi(s)$ satisfies the same properties.

The final argument shows that $\Psi$ is "inward directed", which guarantees that $\Psi$ has a fixed point; see, for instance, [2, Theorem 14.47]. This easily implies that $0 \in \Phi(s)$ for some $s \in \Delta$, as desired.

\section{The core}

In this section we discuss the core of an economy, the set of allocations that are efficient for every subeconomy. This notion was introduced by F. Y. Edgeworth [93].

A coalition is a nonempty set of consumers. The coalition $\{1, \ldots, m\}$ is called the grand coalition. We say that a coalition $S$ blocks (or that it can improve upon) an allocation $\left(x_{1}, \ldots, x_{m}\right)$ if there exists another allocation $\left(y_{1}, \ldots, y_{m}\right)$ such that

1. $\sum_{i \in S} y_{i}=\sum_{i \in S} \omega_{i}$, and

2. $y_{i} \succ_{i} x_{i}$ for each $i \in S$.

In other words, a coalition can improve upon an allocation whenever there is another way of redistributing the resources so that every member of the coalition becomes better off-regardless of what is happening to the consumers of the complementary coalition. Notice that in this terminology an allocation is weakly Pareto efficient if and only if it cannot be blocked by the grand coalition. 
Definition 49 (Edgeworth) $A$ core allocation is an allocation that cannot be blocked by any coalition. The core of an economy $\mathcal{E}$ is the set of core allocations and is denoted $\operatorname{Core}(\mathcal{E})$.

Here are some well known properties of core allocations.

Theorem 50 For exchange economies we have:

1. Every core allocation is weakly Pareto efficient and individually rational.

2. Every Walrasian equilibrium is a core allocation.

It is easy to see that a core allocation need not be a Walrasian equilibrium. Do core allocations exist? By the above discussion, the answer is, of course, yes. Once we know that Walrasian equilibria exist, core allocations also exist. However, it is interesting to know that if we can establish the existence of core allocations in a different manner, then this can be used to present an alternate way of proving the existence of Walrasian equilibria. As we shall see later (Theorem 71), one can establish-in the class of Riesz exchange economies with strictly monotone, continuous, convex, and proper preferences and strictly positive total endowments - that the following two statements are equivalent.

1. Every economy has a Walrasian equilibrium.

2. Every economy has a core allocation.

For the time being, we remark that using game theoretic techniques one can establish the existence of core allocations independently of the existence of Walrasian equilibria. We state this result, which is essentially independently due to $\mathrm{O}$. N. Bondareva and H. E. Scarf.

Theorem 51 (Bondareva-Scarf $[53,190])$ In a Riesz exchange economy assume that:

1. The preferences of each consumer are represented by a monotone and quasiconcave utility function.

2. There exists a consistent locally convex-solid topology on the commodity space $E$ such that each utility function is continuous.

3. The Edgeworth box. $[0, \omega]$ is weakly compact.

Then the core of the economy is a nonempty weakly compact subset of $E^{m}$.

For a proof see $[8$, Theorem 3.3.6, p. 127].

The following example shows that the weak compactness of the Edgeworth box $[0, \omega]$ cannot be dropped from the preceding theorem. 
Example 52 (Aliprantis-Brown-Burkinshaw [4]) Consider a two consumer Riesz exchange economy with the following characteristics.

- Riesz pair: $\langle C[0,1], c a[0,1]\rangle$.

- Consumer 1: Initial endowment $\omega_{1}=1$ (the constant function one) and preference given by the utility function

$$
u_{1}(x)=\int_{0}^{\frac{1}{2}} \sqrt{x(t)} d t+\frac{1}{2} \int_{\frac{1}{2}}^{1} \sqrt{x(t)} d t .
$$

- Consumer 2: Initial endowment $\omega_{2}=1$ and preference given by the utility function

$$
u_{2}(x)=\frac{1}{2} \int_{0}^{\frac{1}{2}} \sqrt{x(t)} d t+\int_{\frac{1}{2}}^{1} \sqrt{x(t)} d t .
$$

Both utility functions are strictly monotone, strictly concave, norm continuous, and norm proper. However, the Edgeworth box $[0, \omega]$ is not weakly compact. It can be shown that this exchange economy has an empty core, see [8, Example 3.3.7, p. 128].

\section{Replication}

Theorems which guarantee that the set of Walrasian equilibrium allocations coincides with the set of core allocations are known in the economic literature as core equivalence theorems. It was conjectured more than one hundred years ago by F. Y. Edgeworth [93] that when the number of consumers in an economy increases, the core of the economy "shrinks" to the set of Walrasian equilibria. The purpose of this section is to make precise this claim by establishing the core equivalence theorem of G. Debreu and H. E. Scarf [87]. To do this, we need to introduce the notion of a replica economy.

Definition 53 The $r$-fold replica of an exchange economy $\mathcal{E}$ is a new exchange economy $\mathcal{E}_{r}$ with the same commodity-price dual pair as $\mathcal{E}$ and $m r$ consumers (indexed by $(i, j)$, $i=1, \ldots, m ; j=1, \ldots, r)$ such that:

- The preference $\succcurlyeq_{i j}$ of consumer $(i, j)$ is the same as the preference of consumer $i$, i.e., $\succcurlyeq_{i j}=\succcurlyeq_{i}$; and

- The initial endowment $\omega_{i j}$ of consumer $(i, j)$ coincides with the initial endowment of consumer $i$, i.e., $\omega_{i j}=\omega_{i}$.

Notice that the total endowment of the $r$-fold replica economy is precisely $r \omega$. The consumers of the form $(i, j)$ for $j=1, \ldots, r$ are all identical and are referred to as consumers of type $i$. An allocation

$$
\left(x_{11}, \ldots, x_{1 r}, x_{21}, \ldots, x_{2 r}, \ldots, x_{m 1}, \ldots, x_{m r}\right)
$$


in an $r$-fold replica economy has the equal treatment property if $x_{i s}=x_{i t}$ for all $i$, $s$, and $t$. That is, consumers of the same type receive identical bundles. Equal treatment allocations can be identified with allocations in the original economy. Indeed, if $\left(x_{1}, \ldots, x_{m}\right)$ is an allocation of some economy, then by letting $x_{i j}=x_{i}$, we can consider $\left(x_{1}, \ldots, x_{m}\right)$ as an allocation in every $r$-fold replica economy.

Lemma 54 In an exchange economy, every Walrasian equilibrium belongs to the core of each replica economy.

Proof: Let $\left(x_{1}, \ldots, x_{m}\right)$ be a Walrasian equilibrium in an exchange economy supported by a price $p$ and consider $\left(x_{1}, \ldots, x_{m}\right)$ as an allocation in some $r$-fold replica. Clearly, $x_{i j}=x_{i} \in B_{i}(p)=B_{i j}(p)$ for all $(i, j)$. Now note that $x \succ_{i j} x_{i j}=x_{i}$ implies $p \cdot x>$ $p \cdot \omega_{i}=p \cdot \omega_{i j}$, and the conclusion follows from part (1) of Lemma 27.

The converse of the preceding result is a reformulation of the celebrated core equivalence theorem of G. Debreu and H. E. Scarf.

Theorem 55 (Debreu-Scarf [87]) In a finite dimensional exchange economy, if preferences are convex, continuous, and strictly monotone, then an allocation is a Walrasian equilibrium if and only if it belongs to the core of every replica economy.

The next theorem takes care of the existence of allocations that belong to the core of every replica economy. In particular, it provides an alternate way of proving the existence of Walrasian equilibria in finite dimensional exchange economies.

Theorem 56 (Debreu-Scarf [87]) Assume that in a Riesz exchange economy:

1. The preferences of each consumer $i$ are represented by a strictly monotone and quasiconcave utility function.

2. There exists a consistent locally convex-solid topology $\tau$ on the commodity space $E$ such that each utility function is continuous.

3. The Edgeworth box $[0, \omega]$ is weakly compact.

Then there is an allocation of $\mathcal{E}$ that belongs to the core of every $r$-fold replica economy of $\mathcal{E}$ (and hence if the economy is also finite dimensional, it is a Walrasian equilibrium).

Proof: Let $\mathcal{E}$,be_a Riesz.exchange economy satisfying the hypotheses stated in the theorem and let $\mathcal{E}_{r}$ denote the $r$-fold replica economy of $\mathcal{E}$. Let $\mathcal{C}_{r}$ be the set of allocations for $\mathcal{E}$ that belong to the core of the economy $\mathcal{E}_{r}$, i.e.,

$$
\mathcal{C}_{r}=\left\{\left(x_{1}, \ldots, x_{m}\right) \in \mathcal{A}:\left(x_{1}, \ldots, x_{m}\right) \in \operatorname{Core}\left(\mathcal{E}_{r}\right)\right\}
$$

Clearly, $\mathcal{C}_{r+1} \subset \mathcal{C}_{r}$ for each $r$ and the intersection $\bigcap_{r=1}^{\infty} \mathcal{C}_{r}$ consists precisely of all allocations for $\mathcal{E}$ that belong to the core of every replica economy. If we establish that each 
$\mathcal{C}_{r}$ is nonempty and weakly closed, then it follows from the finite intersection property that the set $\bigcap_{r=1}^{\infty} \mathcal{C}_{r}$ is nonempty (by Theorem 7 the set $\mathcal{A}$ is weakly compact) and the proof of the theorem is finished. The fact that each $\mathcal{C}_{r}$ is a weakly closed set (and in fact a weakly compact set) is a matter of a straightforward verification. Next, we prove that each $\mathcal{C}_{r}$ is nonempty.

By Theorem 51 the $r$-fold economy has a nonempty core. Let

$$
\left(x_{11}, \ldots, x_{1 r}, x_{21}, \ldots, x_{2 r}, \ldots, x_{m 1}, \ldots, x_{m r}\right)
$$

be a core allocation for the economy $\mathcal{E}_{r}$. We claim that $x_{i j} \sim_{i} x_{i k}$ for $j, k=1, \ldots, r$ and $i=1, \ldots, m$, i.e., we claim that no consumer strictly prefers his bundle to that of another consumer of the same type. To see this, we can assume (by rearranging the consumers of each type) that $x_{i j} \succcurlyeq_{i} x_{i 1}$ for each $i$ and $j$. Let

$$
y_{i 1}=\frac{1}{n} \sum_{j=1}^{m} x_{i j}, \quad i=1, \ldots, m,
$$

and note that $y_{i 1} \succcurlyeq_{i} x_{i 1}$ for all $i$. If for some $1 \leqslant i \leqslant m$ and some $1 \leqslant s \leqslant r$ we have $x_{i s} \succ_{i} x_{i 1}$, then it follows that $y_{i 1} \succ_{i} x_{i 1}$. Now if each consumer $(i, 1)$ gets the bundle $y_{i 1}$, then the coalition $\{(i, 1): i=1, \ldots, m\}$ blocks the original allocation $\left(x_{i j}\right)$, which is a contradiction. To finish the proof note that $\left(y_{11}, y_{21}, \ldots, y_{m 1}\right)$ is an allocation of the economy $\mathcal{E}$ satisfying $y_{i 1} \succcurlyeq_{i} x_{i j}$ for all $i$ and $j$, so $\left(y_{11}, y_{21}, \ldots, y_{m 1}\right) \in \mathcal{C}_{r}$.

The parenthetical part follows immediately from Theorem 55 .

\section{Edgeworth equilibria}

We saw in the preceding section that in certain finite dimensional exchange economies the Walrasian equilibria coincide with the allocations that belong to the core of every replica economy. Also, Theorem 56 provides some conditions that guarantee the existence of such allocations. Our purpose here is to isolate and study the allocations that belong to the core of every replica economy. In general, as we shall see, these allocations need not be Walrasian equilibria or even quasiequilibria.

Definition 57 (Aliprantis-Brown-Burkinshaw [5]) An allocation in an exchange economy is an Edgeworth equilibrium if it belongs to the core of every replication of the economy.

Obviously, every Edgeworth equilibrium is a core allocation (and hence it is individually rational and weakly Pareto efficient). The notion of Edgeworth equilibrium is a price free notion in the following sense. If the commodity space $X$ is a Riesz space, then all economic activity takes place inside the Edgeworth box $[0, \omega]$. So in this case, if $\left(x_{1}, \ldots, x_{m}\right)$ is an Edgeworth equilibrium, then the allocation $\left(x_{1}, \ldots, x_{m}\right)$ remains an 
Edgeworth equilibrium in any exchange economy whose commodity space is a Riesz subspace of $X$ containing the principal ideal $E_{\omega}$ (regardless of the nature of the price space $\left.X^{\prime}\right)$.

It is clear that every Walrasian equilibrium is automatically an Edgeworth equilibrium. As we shall see in the next section, the Edgeworth equilibria are precisely the allocations that are Walrasian equilibria with respect to the Riesz pair $\left\langle E_{\omega}, E_{\omega}^{\prime}\right\rangle$. The fact that a Walrasian equilibrium with respect to a price of $E_{\omega}^{\prime}$ is an Edgeworth equilibrium is easy to prove. We start by stating this result and leave its straightforward proof as an exercise.

Lemma 58 Let $\left(x_{1}, \ldots, x_{m}\right)$ be an allocation in a Riesz commodity exchange economy. If there exists a price $0<p \in E_{\omega}^{\prime}$ (not necessarily lying in $X^{\prime}$ ) such that $x \succ_{i} x_{i}$ in $E_{\omega}^{+}$ implies $p \cdot x>p \cdot \omega_{i}$, then the allocation $\left(x_{1}, \ldots, x_{m}\right)$ is an Edgeworth equilibrium.

As an illustration of Lemma 58, we present an example due to L. E. Jones.

Example 59 (Jones [119]) Consider an exchange economy with commodity space $X=$ $L_{1}[0,1]$ and two consumers with initial endowments $\omega_{1}=\omega_{2}=\frac{1}{2} \chi_{[0,1]}$ and preferences represented by the utility functions

$$
u_{1}(x)=\int_{0}^{1} t x(t) d t \quad \text { and } \quad u_{2}(x)=\int_{0}^{1}(1-t) x(t) d t .
$$

Note that both utility functions are strictly monotone and convex (in fact linear). If the functions $z_{1}(t)=t$ and $z_{2}(t)=1-t$ belong to $X^{\prime}$, then $u_{1}$ and $u_{2}$ are also weakly continuous.

Clearly, $\omega=1$ and if we put $x_{1}=\chi_{\left(\frac{1}{2}, 1\right]}$ and $x_{2}=\chi_{\left[0, \frac{1}{2}\right.}$, then $\left(x_{1}, x_{2}\right)$ is an allocation. Let $p(t)=\max \{t, 1-t\}$. We claim that $x \succ_{i} x_{i}$ implies $p \cdot x>p \cdot \omega_{i}$. To see this, note first that $p \cdot x_{1}=p \cdot x_{2}=p \cdot \omega_{1}=p \cdot \omega_{2}=\frac{3}{8}$. Now if $x \succ_{1} x_{1}$, then

$$
p \cdot x=\int_{0}^{1} p(t) x(t) d t \geqslant \int_{0}^{1} t x(t) d t=u_{1}(x)>u_{1}\left(x_{1}\right)=\int_{\frac{1}{2}}^{1} t d t=\frac{3}{8}=p \cdot \omega_{1} .
$$

Similarly, $x \succ_{2} x_{2}$ implies $p \cdot x>p \cdot \omega_{2}$. Hence, $\left(x_{1}, x_{2}\right)$ is a Walrasian equilibrium with respect to the price $p$ so by Lemma $58,\left(x_{1}, x_{2}\right)$ is an Edgeworth equilibrium. In particular, $\left(x_{1}, x_{2}\right)$ is also a weakly Pareto efficient (and hence a Pareto efficient) allocation.

We now show that $p$ is (up to a constant) the only price that supports the allocation $\left(x_{1}, x_{2}\right)$. To this end, let $q$ be a price supporting $\left(x_{1}, x_{2}\right)$. By Lemma 12 , we know that $q$ is a positive linear functional. This implies that $q$ belongs to the order dual of $L_{1}[0,1]$, i.e., $0<q \in L_{\infty}[0,1]$.

Next, we claim that $q$ is strictly positive. To see this, let $A=\left\{t \in\left[0, \frac{1}{2}\right]: q(t)=0\right\}$. If $A$ has positive Lebesgue measure, then for some $k>0$ we have $u_{1}\left(k \chi_{A}\right)=k \int_{A} t d t>$ 
$u_{1}\left(x_{1}\right)$, so by the supportability of $q$, we get

$$
0=q \cdot\left(k \chi_{A}\right) \geqslant q \cdot x_{1}=\int_{\frac{1}{2}}^{1} q(t) d t \geqslant 0,
$$

from which it follows that $q=0$ a.e. on $\left[\frac{1}{2}, 1\right]$. Replacing $A$ by $\left[\frac{1}{2}, 1\right]$, we see that $q=0$ a.e. also holds on $\left[0, \frac{1}{2}\right]$, a contradiction. A similar argument shows that if the set $\left\{t \in\left[\frac{1}{2}, 1\right]: q(t)=0\right\}$ has positive Lebesgue measure, then $q=0$ a.e. So $q(t)>0$ for almost all $t$.

If $A$ is a measurable subset of $\left[\frac{1}{2}, 1\right]$ of positive Lebesgue measure, then $\frac{u_{1}\left(x_{1}\right)}{u_{1}\left(\chi_{A}\right)} \chi_{A} \succcurlyeq_{1} x_{1}$. Therefore, $\frac{u_{1}\left(x_{1}\right)}{u_{1}\left(\chi_{A}\right)} \int_{A} q(t) d t \geqslant \int_{\frac{1}{2}}^{1} q(t) d t$, and consequently

$$
\int_{A} q(t) d t \geqslant\left[\frac{1}{u_{1}\left(x_{1}\right)} \int_{\frac{1}{2}}^{1} q(t) d t\right] \int_{A} t d t
$$

for each measurable subset $A$ of $\left[\frac{1}{2}, 1\right]$. This implies $q(t) \geqslant c t$ for almost all $t \in\left[\frac{1}{2}, 1\right]$, where $c=\frac{1}{u_{1}\left(x_{1}\right)} \int_{\frac{1}{2}}^{1} q(t) d t$. We claim that $q(t)=c t$ for almost all $t \in\left[\frac{1}{2}, 1\right]$. To see this, assume that $\left\{t \in\left[\frac{1}{2}, 1\right]: q(t)>c t\right\}$ has positive Lebesgue measure. Then

$$
\int_{\frac{1}{2}}^{1} q(t) d t>c \int_{\frac{1}{2}}^{1} t d t=c u_{1}\left(x_{1}\right)=\int_{\frac{1}{2}}^{1} q(t) d t,
$$

which is impossible. Hence $q(t)=c t$ for almost all $t \in\left[\frac{1}{2}, 1\right]$. Similarly, $q(t)=c^{*}(1-t)$ for for almost all $t \in\left[0, \frac{1}{2}\right]$.

Next, we show that $c=c^{*}$. Recall that $u_{1}\left(x_{1}\right)=u_{2}\left(x_{2}\right)=\frac{3}{8}$. For $n \geqslant 2$ let $x=\frac{3}{4} \cdot \frac{n^{2}}{n-1} \chi_{\left[\frac{1}{2}-\frac{1}{n}, \frac{1}{2}\right]}$ and note that $u_{1}(x)=\frac{3}{8}$. Thus, $x \succeq_{1} x_{1}$, so support by $q$ implies $q \cdot x \geqslant q \cdot x_{1}$. But $q \cdot x=\frac{3}{8} \frac{n+1}{n-1} c^{*}$ and $q \cdot x_{1}==\frac{3}{8} c$ so that $\frac{3}{8} \frac{n+1}{n-1} c^{*} \geqslant \frac{3}{8} c$. Letting $n \rightarrow \infty$, we get $c^{*} \geqslant c$. Similarly, if $y=\frac{3}{4} \frac{n^{2}}{n-1} \chi_{\left[\frac{1}{2}, \frac{1}{2}+\frac{1}{n}\right]}$, then $u_{2}(y)=\frac{3}{8}$ and so $y \succeq_{2} x_{2}$. Support by $q$ implies $q \cdot y=\frac{3}{8} \frac{n+1}{n-1} c \geqslant q \cdot x_{2}=\frac{3}{8} c^{*}$ and by letting $n \rightarrow \infty$, we get $c \geqslant c^{*}$. Thus, $c=c^{*}$. Consequently, $q=c p$ proving that $p$ is the only price that supports $\left(x_{1}, x_{2}\right)$.

If the price space $X^{\prime}$ does not contain $p$ (for instance, let $X^{\prime}=C^{1}[0,1]$, the vector subspace of $L_{\infty}[0,1]$ consisting of all continuously differentiable functions on $[0,1]$ ), then the allocation $\left(x_{1}, x_{2}\right)$ is an Edgeworth equilibrium with respect to $\left\langle X, X^{\prime}\right\rangle$ but not a Walrasian equilibrium.

The preceding example can be employed to illustrate Theorem 24, which asserts that in a lattice exchange economy (with the standard hypotheses) every weakly efficient allocation can be supported by prices. Since a linear preference (i.e., a preference that is represented by a utility function of the form $u(x)=p \cdot x$ for some linear functional $p$ on $E$ ) is proper if and only if it is continuous, we see that in the setting of the preceding example, the hypotheses of Theorem 24 guarantee that the linear functionals represented 
by the functions $t$ and $1-t$ must lie in $X^{\prime}$. In particular, if the price space $X^{\prime}$ is a Riesz subspace of the order dual $L_{\infty}[0,1]$ of $L_{1}[0,1]$, then $X^{\prime}$ must contain the Riesz subspace generated by 1 and $t$ (which coincides with the vector space of all continuous piecewise linear functions). Therefore, if the hypotheses of Theorem 24 are satisfied, then $X^{\prime}$ contains all the continuous piecewise linear functions.

The next example illustrates the fact that Edgeworth equilibria need not be supported by prices in the given price space.

Example 60 Consider a two consumer exchange economy with two identical consumers; each has an initial endowment $\omega>0$ and a convex preference $\succcurlyeq$ defined on a common convex consumption set. Then, we claim that the allocation $(\omega, \omega)$ is an Edgeworth equilibrium. To see this, assume in some $n$-fold replica economy there exist an allocation $\left\{x_{i j}: i=1, \ldots, m ; j=1, \ldots, n\right\}$ and a coalition $S$ such that

1. $\sum_{(i, j) \in S} x_{i j}=k \omega$ (where $k$ is the number of consumers in $S$ ); and

2. $x_{i j} \succ \omega$ for each $(i, j) \in S$.

Hence, if $\left(i_{0}, j_{0}\right) \in S$ satisfies $x_{i j} \succcurlyeq x_{i_{0} j_{0}}$ for each $(i, j) \in S$, then by the convexity of $\succcurlyeq$, we see that

$$
\omega=\frac{1}{k} \sum_{(i, j) \in S} x_{i j} \succcurlyeq x_{i_{0} j_{0}} \succ \omega
$$

which is impossible. Therefore, $(\omega, \omega)$ is an Edgeworth equilibrium. However, such an equilibrium need not be supported by any price in $X^{\prime}$; see Examples 15 and 35.

With any allocation $\left(x_{1}, \ldots, x_{m}\right)$ we define the two nonempty sets

$$
F_{i}=\left\{x \in X_{i}: x \succcurlyeq_{i} x_{i}\right\} \quad \text { and } \quad G_{i}=F_{i}-\omega_{i}=\left\{x \in X: x+\omega_{i} \succcurlyeq_{i} x_{i}\right\} \text {. }
$$

If the preferences are convex, then the $F_{i}$ and $G_{i}$ are all convex sets, so the convex hull $\mathbf{G}$ of the union of the $G_{i}$ is given by

$$
\mathbf{G}=\operatorname{co}\left(\bigcup_{i=1}^{m} G_{i}\right)=\left\{\sum_{i=1}^{m} \lambda_{i} g_{i}: g_{i} \in G_{i}, \lambda_{i} \geqslant 0 \text { for each } i, \text { and } \sum_{i=1}^{m} \lambda_{i}=1\right\} \text {. }
$$

When the allocation is an Edgeworth equilibrium the convex set $\mathbf{G}$ satisfies an important approximation property. This approximation property is the key property in establishing several "core equivalence" type theorems.

Lemma $61^{2}$ Consider a Riesz exchange economy such that:

\footnotetext{
${ }^{2}$ This lemma was first proved by B. Peleg and M. Yaari [177] for a special case. In its present form the lemma was established by C. D. Aliprantis, D. J. Brown and O. Burkinshaw [5]. Its proof demonstrates the importance and usefulness of the Riesz space structures.
} 


\section{Preferences are convex and weakly upper semicontinuous.}

2. The Edgeworth box $[0, \omega]$ is weakly compact.

3. The total endowment $\omega$ is desirable for each consumer.

If $\left(x_{1}, \ldots, x_{m}\right)$ is an Edgeworth equilibrium, then for each $\varepsilon>0$ we have $0 \notin \varepsilon \omega+\overline{\mathbf{G}}$ (where the bar denotes weak closure).

Proof: Let $\left\langle E, E^{\prime}\right\rangle$ denote the Riesz pair of the exchange economy. Fix an Edgeworth equilibrium $\left(x_{1}, \ldots, x_{m}\right)$ and let $\varepsilon>0$. Also, assume by way of contradiction that $0 \in \varepsilon \omega+\overline{\mathbf{G}}$ or $-\varepsilon \omega \in \overline{\mathbf{G}}$.

Let $\tau$ denote the absolute weak topology on $E$, i.e., let $\tau=|\sigma|\left(E, E^{\prime}\right)$. Since $\mathbf{G}$ is convex and $\tau$ is a consistent topology on $E$, it follows that the weak and $\tau$ closures of $\mathbf{G}$ coincide. Hence, there exists a net $\left\{g_{\alpha}\right\}$ in $\mathbf{G}$ such that $g_{\alpha} \stackrel{\tau}{\rightarrow}-\varepsilon \omega$.

For each $\alpha$ choose $g_{i}^{\alpha} \in G_{i}, \lambda_{i}^{\alpha} \geqslant 0(i=1, \ldots, m)$ with $\sum_{i=1}^{m} \lambda_{i}^{\alpha}=1$ and $g_{\alpha}=$ $\sum_{i=1}^{m} \lambda_{i}^{\alpha} g_{i}^{\alpha}$. By passing to a subnet, we can assume that $\lambda_{i}^{\alpha} \rightarrow \lambda_{i} \geqslant 0$ for each $i$. From $\sum_{i=1}^{m} \lambda_{i}=1$, we see that the set $S=\left\{n \in\{1, \ldots, m\}: \lambda_{n}>0\right\}$ is nonempty. By passing to a subnet again, we can assume that there exists some $\delta>0$ such that $\lambda_{n}^{\alpha} \geqslant \frac{1}{\delta}$ for all $n \in S$ and all $\alpha$.

Next, for each $i$ write $g_{i}^{\alpha}=f_{i}^{\alpha}-\omega_{i}$. We claim that for each $n \in S$ the net $\left\{f_{n}^{\alpha}\right\}$ has a weakly convergent subnet in $E$. To this end, fix $n \in S$. Then

$$
\begin{aligned}
0 \leqslant \lambda_{n}^{\alpha} f_{n}^{\alpha} & \leqslant \sum_{i=1}^{m} \lambda_{i}^{\alpha} f_{i}^{\alpha}=\sum_{i=1}^{m} \lambda_{i}^{\alpha}\left(g_{i}^{\alpha}+\omega_{i}\right)=\sum_{i=1}^{m} \lambda_{i}^{\alpha} g_{i}^{\alpha}+\sum_{i=1}^{m} \lambda_{i}^{\alpha} \omega_{i} \\
& =g_{\alpha}+\sum_{i=1}^{m} \lambda_{i}^{\alpha} \omega_{i} \leqslant g_{\alpha}+\omega \leqslant\left(g_{\alpha}+\varepsilon \omega\right)+\omega
\end{aligned}
$$

and consequently

$$
0 \leqslant f_{n}^{\alpha} \leqslant \frac{1}{\lambda_{n}^{\alpha}}\left(g_{\alpha}+\varepsilon \omega\right)+\frac{1}{\lambda_{n}^{\alpha}} \omega \leqslant \delta\left(g_{\alpha}+\varepsilon \omega\right)+\delta \omega .
$$

Since $[0, \delta \omega]=\delta[0, \omega]$ is (by our hypothesis) a weakly compact order interval and $g_{\alpha}+$ $\varepsilon \omega \stackrel{\tau}{\rightarrow} 0$, it follows that $\left\{f_{n}^{\alpha}\right\}$ has a weakly convergent subnet. So by passing to an appropriate subnet, we can assume that $f_{n}^{\alpha} \underset{\alpha}{\vec{\alpha}} f_{n}$ in $E$ for each $n \in S$. By the weak upper semicontinuity of preferences each $F_{n}$ is weakly closed, so $f_{n} \in F_{n}$ (i.e., $f_{n} \succcurlyeq_{n} x_{n}$ ) for each $n \in S$. From

$$
g_{\alpha}=\sum_{i=1}^{m} \lambda_{i}^{\alpha}\left(f_{i}^{\alpha}-\omega_{i}\right) \geqslant \sum_{n \in S} \lambda_{n}^{\alpha} f_{n}^{\alpha}-\sum_{i=1}^{m} \lambda_{n}^{\alpha} \omega_{n} \underset{\alpha}{\stackrel{w}{\longrightarrow}} \sum_{n \in S} \lambda_{n} f_{n}-\sum_{n \in S} \lambda_{n} \omega_{n}
$$


and the fact that $E^{+}$is weakly closed, we see that

$$
-\varepsilon \omega=w-\lim _{\alpha} g_{\alpha} \geqslant \sum_{n \in S} \lambda_{n} f_{n}-\sum_{n \in S} \lambda_{n} \omega_{n}
$$

Rearranging, we get

$$
\sum_{n \in S} \lambda_{n}\left(f_{n}+\varepsilon \omega\right) \leqslant \sum_{n \in S} \lambda_{n} \omega_{n}
$$

By the desirability of $\omega$, we have $f_{n}+\varepsilon \omega \succ_{n} f_{n} \succcurlyeq_{n} x_{n}$ for each $n \in S$. Finally, using $(\star)$ an easy argument shows that $\left(x_{1}, \ldots, x_{m}\right)$ can be blocked in some replica economy, which is a contradiction. The proof of the lemma is now complete.

\section{Edgeworth equilibria and quasiequilibria}

Here we relate the Edgeworth equilibria to Walrasian equilibria and quasiequilibria. The results in this section are due to C. D. Aliprantis, D. J. Brown and O. Burkinshaw [5].

We start by introducing the notion of an approximate quasiequilibrium.

Definition 62 An allocation $\left(x_{1}, \ldots, x_{m}\right)$ in an exchange economy is an approximate quasiequilibrium if for each $\varepsilon>0$ there exists some nonzero price $p \in X^{\prime}$ (depending upon $\varepsilon$ ) such that

1. $p \cdot \omega=1$; and

2. $x \succcurlyeq_{i} x_{i}$ implies $p \cdot x \geqslant p \cdot \omega_{i}-\varepsilon$.

In a Riesz commodity exchange economy with at least one consumer having a monotone preference, say the first one, every price $p$ that satisfies properties (1) and (2) of the above definition is necessarily a positive price. Indeed, if $x \geqslant 0$, then $x_{1}+\delta^{-1} x \succcurlyeq_{1} x_{1}$ for each $\delta>0$. Hence, $p \cdot x_{1}+\delta^{-1} p \cdot x \geqslant p \cdot \omega_{1}-\varepsilon$ or $p \cdot x \geqslant \delta\left(p \cdot \omega_{1}-p \cdot x_{1}-\varepsilon\right)$ for each $\delta>0$ from which it follows that $p \cdot x \geqslant 0$.

Lemma 63 Consider a Riesz exchange economy such that:

1. Preferences are convex and weakly upper semicontinuous.

2. The Edgeworth box $[0, \omega]$ is weakly compact.

3. The total endowment $\omega$ is desirable for each consumer.

Then every Edgeworth equilibrium is an approximate quasiequilibrium. 
Proof: Consider an exchange economy satisfying the hypotheses, let $\left(x_{1}, \ldots, x_{m}\right)$ be an Edgeworth equilibrium, and let $\varepsilon>0$. By Lemma 61 , we know that $0 \notin \varepsilon \omega+\overline{\mathbf{G}}$, where

$$
\mathbf{G}=\operatorname{co}\left(\bigcup_{i=1}^{m} G_{i}\right)=\left\{\sum_{i=1}^{m} \lambda_{i} g_{i}: g_{i} \in G_{i}, \lambda_{i} \geqslant 0 \text { for each } i, \text { and } \sum_{i=1}^{m} \lambda_{i}=1\right\}
$$

and $G_{i}=\left\{x \in X: x+\omega_{i} \succcurlyeq_{i} x_{i}\right\}$. So by the Separating Hyperplane Theorem [2, Theorem 4.54, p. 147], there exists some price $p \in E^{\prime}$ satisfying

$$
p \cdot(g+\varepsilon \omega)>0 \text { for all } g \in \mathbf{G} \text {. }
$$

Clearly $p \cdot \omega>0$. Indeed, $x_{i}-\omega_{i} \in G_{i}$, so $0=\sum_{i=1}^{m} \frac{1}{m}\left(x_{i}-\omega_{i}\right) \in \mathbf{G}$, and thus $p \cdot \omega=\frac{1}{\varepsilon} p \cdot(0+\varepsilon \omega)>0$.

Replacing $p$ by $\frac{p}{p \cdot \omega}$, we can assume $p \cdot \omega=1$. If $x \succcurlyeq_{i} x_{i}$, then $x-\omega_{i} \in \mathbf{G}$, so $p \cdot\left(x-\omega_{i}+\varepsilon \omega\right)>0$, from which it follows that $p \cdot x \geqslant p \cdot \omega_{i}-\varepsilon$, as desired.

The approximate quasiequilibria are Walrasian equilibria for a subeconomy of the original economy.

Lemma 64 If $\left(x_{1}, \ldots, x_{m}\right)$ is an approximate quasiequilibrium for a Riesz exchange economy, then there exists a positive linear functional $p$ on $E_{\omega}$ (the ideal generated by the total endowment $\omega$ ) such that:

1. $p \cdot \omega=1$; and

2. $x \succcurlyeq_{i} x_{i}$ in $E_{\omega}^{+}$implies $p \cdot x \geqslant p \cdot \omega_{i}$.

That is, every approximate quasiequilibrium is a quasiequilibrium with respect to another Riesz economy whose consumer characteristics are the same as the original ones and with Riesz pair $\left\langle E_{\omega}, E_{\omega}^{\prime}\right\rangle$.

Proof: Let $\left(x_{1}, \ldots, x_{m}\right)$ be an approximate quasiequilibrium for a Riesz exchange economy. For each natural number $n$ choose some $0 \leqslant p_{n} \in E^{\prime}$ with $p_{n} \cdot \omega=1$ and such that

$$
x \succcurlyeq_{i} x_{i} \text { in } E^{+} \quad \text { implies } \quad p_{n} \cdot x \geqslant p_{n} \cdot \omega_{i}-\frac{1}{n}
$$

Now consider each $p_{n}$ restricted to $E_{\omega}$. The condition $p_{n} \cdot \omega=1$ implies that $p_{n}$ as a functional on the-nermed space $\left(E_{\omega} ;\left\|_{*}\right\|_{\infty}\right)$ has norm one so by the Alaoglu Compactness Theorem [2, Theorem 4.79], the sequence $\left\{p_{n}\right\}$ has a $w^{*}$-accumulation point, say $p$. Then, $p$ is a positive functional on $E_{\omega}$ (although it need not be defined on all of $E$ ), $p \cdot \omega=1$, and $x \succcurlyeq_{i} x_{i}$ in $E_{\omega}^{+}$implies $p \cdot x \geqslant p \cdot \omega_{i}$.

And now we come to an important result that describes the relationships between the various equilibria notions introduced before. 
Theorem 65 (Aliprantis-Brown-Burkinshaw [5]) Consider a Riesz exchange economy with Riesz pair $\left\langle E, E^{\prime}\right\rangle$ such that:

a. Preferences are convex, continuous for some consistent locally convex-solid topology on $E$, and monotone on $E_{\omega}^{+}$.

b. The Edgeworth box $[0, \omega]$ is weakly compact.

c. The total endowment $\omega$ is desirable for each consumer.

Then for an allocation the following statements are equivalent.

1. The allocation is an Edgeworth equilibrium.

2. The allocation is an approximate quasiequilibrium.

3. The allocation is a Walrasian equilibrium for the Riesz exchange economy with the same preferences and endowments and Riesz pair $\left\langle E_{\omega}, E_{\omega}^{\prime}\right\rangle$.

Proof: Consider an exchange economy that satisfies the properties stated in the theorem and let $\left(x_{1}, \ldots, x_{m}\right)$ be an allocation.

$(1) \Longrightarrow(2)$ This is an immediate consequence of Lemma 63 .

$(2) \Longrightarrow(3)$ By Lemma 64 the allocation $\left(x_{1}, \ldots, x_{m}\right)$ is a quasiequilibrium with respect to the Riesz pair $\left\langle E_{\omega}, E_{\omega}^{\prime}\right\rangle$. Since preferences are strictly monotone on $E_{\omega}^{+}$, it follows from Corollary 32 that $\left(x_{1}, \ldots, x_{m}\right)$ is a Walrasian equilibrium with respect to $\left\langle E_{\omega}, E_{\omega}^{\prime}\right\rangle$.

$(3) \Longrightarrow(1)$ This is an immediate consequence of Lemma 58.

The next example illustrates the preceding theorem.

Example 66 Consider once more the Riesz exchange economy introduced in Example 15. In Example 35, we established that $\left(\omega_{1}, \omega_{2}\right)$ was a Walrasian equilibrium with respect to the Riesz pair $\left\langle E_{\omega}, E_{\omega}^{\prime}\right\rangle$ (but not a Walrasian equilibrium with respect to the original Riesz pair $\left.\left\langle\ell_{1}, \ell_{\infty}\right\rangle\right)$. So according to Theorem 65, $\left(\omega_{1}, \omega_{2}\right)$ is an Edgeworth equilibrium or, equivalently, an approximate quasiequilibrium. We now demonstrate the existence of the prices that support $\left(\omega_{1}, \omega_{2}\right)$ as an approximate quasiequilibrium. So let $\varepsilon>0$.

Fix some $k$ such that $\sum_{n=k}^{\infty} \frac{1}{2^{n}}<\frac{\varepsilon}{2}$ and consider the $\ell_{\infty}$-price $q=\left(2,2^{2}, \ldots, 2^{k}, 1,1,1, \ldots\right)$. Then $q$ satisfies the following properties.

- $u\left(\omega_{1}\right) \geqslant q \cdot \omega_{1}$. 
To see this, note that

$$
u\left(\omega_{1}\right)=\sum_{n=1}^{\infty} \frac{1}{2^{n+1}} \geqslant \sum_{n=1}^{k} \frac{1}{2^{n+1}}+\sum_{n=k+1}^{\infty} \frac{1}{2^{2 n+1}}=q \cdot \omega_{1} .
$$

- $q \cdot x \geqslant u(x)-\frac{\varepsilon}{2}$ for each $0 \leqslant x \in \ell_{1}$.

We proved in Example 35 that $2^{n} t \geqslant \varphi_{n}(t)$ for each $t \geqslant 0$. Moreover, from the definition of $\varphi_{n}$, we see that $t+\frac{1}{2^{n}} \geqslant \varphi_{n}(t)$ for all $t \geqslant 0$. So if $x=\left(x_{1}, x_{2}, \ldots\right) \in \ell_{1}^{+}$, then

$$
\begin{aligned}
q \cdot x & =\sum_{n=1}^{k} 2^{n} x_{n}+\sum_{n=k+1}^{\infty} x_{n}=\sum_{n=1}^{k} 2^{n} x_{n}+\sum_{n=k+1}^{\infty}\left(x_{n}+\frac{1}{2^{n}}\right)-\sum_{n=k+1}^{\infty} \frac{1}{2^{n}} \\
& \geqslant \sum_{n=1}^{k} \varphi_{n}\left(x_{n}\right)+\sum_{n=k+1}^{\infty} \varphi_{n}\left(x_{n}\right)-\frac{\varepsilon}{2}=u(x)-\frac{\varepsilon}{2} .
\end{aligned}
$$

- If $x \succcurlyeq_{1} \omega_{1}$, then $q \cdot x \geqslant q \cdot \omega_{1}-\frac{\varepsilon}{2}$.

Indeed, if $x \succcurlyeq_{1} \omega_{1}$, then from the preceding two properties we see that

$$
q \cdot x \geqslant u(x)-\frac{\varepsilon}{2} \geqslant u\left(\omega_{1}\right)-\frac{\varepsilon}{2} \geqslant q \cdot \omega_{1}-\frac{\varepsilon}{2} .
$$

- If $p=\frac{q}{q \cdot \omega} \in E_{\omega}^{\prime}$, then $p \cdot \omega=1$ and $x \succcurlyeq_{1} \omega_{1}$ implies $p \cdot x \geqslant p \cdot \omega_{1}-\varepsilon$.

Clearly, $p \cdot \omega=1$, where $\omega=\omega_{1}+\omega_{2}=\left(\frac{1}{2^{2}}, \frac{1}{2^{4}}, \ldots, \frac{1}{2^{2 n}}, \ldots\right)$, and $q \cdot \omega>\frac{1}{2}$. Now if $x \succcurlyeq_{1} \omega_{1}$, then from the last property we get

$$
p \cdot x=\frac{q \cdot x}{q \cdot \omega} \geqslant \frac{q \cdot \omega_{1}}{q \cdot \omega}-\frac{\varepsilon}{2 q \cdot \omega} \geqslant p \cdot \omega_{1}-\varepsilon .
$$

Therefore, the price $p$ is " $\varepsilon$-supporting" the Edgeworth equilibrium $\left(\omega_{1}, \omega_{2}\right)$.

Note that statements (1) and (3) of Theorem 65 remain equivalent without the assumption that the Edgeworth box $[0, \omega]$ is weakly compact. Specifically, we have the following companion result to Theorem 65 .

Theorem 67 (Aliprantis-Brown-Burkinshaw [5]) In a Riesz exchange economy, suppose that preferences are convex, continuous for some consistent linear topology on $E$, and strictly monotone on $E_{\omega}^{+}$. Then the following statements about an allocation are equivalent.

1. The allocation is an Edgeworth equilibrium.

2. The allocation is an approximate quasiequilibrium with respect to the Riesz pair $\left\langle E_{\omega}, E_{\omega}^{\prime}\right\rangle$.

3. The allocation is a Walrasian equilibrium with respect to the Riesz pair $\left\langle E_{\omega}, E_{\omega}^{\prime}\right\rangle$. 
For a proof see $[8$, Theorem 3.4 .18 , p. 145].

We close the section by establishing an important equilibrium existence result of T. F. Bewley [46, 47]. Consider the Riesz pair $\left\langle L_{\infty}, L_{1}\right\rangle$, where $L_{\infty}=L_{\infty}(\mu)$ and $L_{1}=L_{1}(\mu)$ for some $\sigma$-finite measure $\mu$. We know that $\left\langle L_{\infty}, L_{1}\right\rangle$ is a symmetric Riesz pair and that the Mackey topology $\tau\left(L_{\infty}, L_{1}\right)$ is locally solid and order continuous; see Theorem [2, Theorem 7.32]. Moreover, by [2, Theorem 7.30], we also know that the only order continuous prices on $L_{\infty}$ are the prices in $L_{1}$.

Theorem 68 (Bewley [47]) Consider a Riesz exchange economy having Riesz pair $\left\langle L_{\infty}, L_{1}\right\rangle$ and whose preferences are represented by strictly monotone, quasiconcave, and Mackey continuous utility functions.

If the total endowment $\omega$ is bounded away from zero (i.e., if there exists some $\varepsilon>0$ such that $\omega(x) \geqslant \varepsilon$ for $\mu$-almost all $x)$, then the economy has a Walrasian equilibrium.

Proof: Start by observing that since $\omega$ is bounded away from zero, we have $E_{\omega}=L_{\infty}$. Now, by Theorem 56, the economy has an Edgeworth equilibrium which, by Theorem 65, it is supported as a Walrasian equilibrium by a price $0<p \in E_{\omega}^{\prime}=L_{\infty}^{\prime}$. Since the order interval $[0, \omega]$ is weakly (i.e. $\sigma\left(L_{\infty}, L_{1}\right)$-)compact, it follows from Theorem $34(2)$ that $p$ is order continuous on $L_{\infty}$, and thus $p \in L_{1}$. Therefore, the economy has a Walrasian equilibrium.

We close the section by mentioning that approximate equilibria in connection with the welfare theorems have been studied by C. D. Aliprantis and O. Burkinshaw [12], R. A. Becker, H. Bercovici, and C. Foias [39], and M. A. Khan and R. Vohra [128].

\section{Edgeworth equilibria and properness}

In the previous sections, we saw that an Edgeworth equilibrium need not be a quasiequilibrium. However, if the economy has uniformly proper preferences, then every Edgeworth equilibrium is a quasiequilibrium. This is the basic result of this section.

Theorem 69 (Aliprantis-Brown-Burkinshaw [5]) Consider a Riesz exchange economy such that each preference is monotone, convex, and uniformly proper for a consistent locally convex-solid topology $\tau$ on the commodity space. Then every Edgeworth equilibrium is a quasiequilibrium (with respect to the Riesz pair $\left\langle E, E^{\prime}\right\rangle$ ).

Proof: Consider a Riesz exchange economy having the properties stated in the theorem and let $\left(x_{1}, \ldots, x_{m}\right)$ be an Edgeworth equilibrium. As before, define

$$
F_{i}=\left\{x \in E^{+}: x \succcurlyeq_{i} x_{i}\right\} \text { and } G_{i}=F_{i}-\omega_{i}=\left\{x \in E: x+\omega_{i} \succcurlyeq_{i} x_{i}\right\},
$$

and let $\mathbf{G}=\operatorname{co}\left(\bigcup_{i=1}^{m} G_{i}\right)$. 
Since each preference is uniformly $\tau$-proper, for each $i$ there exist a convex, solid, open $\tau$-neighborhood $V_{i}$ of zero and some $v_{i}>0$ such that $x-\alpha v_{i}+z \succcurlyeq_{i} x$ in $E^{+}$with $\alpha>0$ imply $z \notin \alpha V_{i}$. Put $V=\bigcap_{i=1}^{m} V_{i}$ and $v=\sum_{i=1}^{m} v_{i}$, and define the nonempty, convex and $\tau$-open cone $\Gamma$ of $E$ by

$$
\Gamma=\left\{\alpha w: \alpha>0 \text { and } w \in E \text { satisfies } w+v \in \frac{1}{2} V\right\} .
$$

We claim that $\Gamma \cap \mathbf{G}=\varnothing$. If this is the case, then by the Separating Hyperplane Theorem [2, Theorem 4.46, p. 143] there exists some nonzero price $p \in E^{\prime}$ satisfying $p \cdot g \geqslant p \cdot \gamma$ for all $g \in \mathbf{G}$ and all $\gamma \in \Gamma$. Since $\gamma \in \Gamma$ implies $\alpha \gamma \in \Gamma$ for all $\alpha>0$, we see that $p \cdot g \geqslant 0$ for each $g \in \mathbf{G}$. Now, if $x \succcurlyeq_{i} x_{i}$, then $x-\omega_{i} \in \mathbf{G}$, so $p \cdot\left(x-\omega_{i}\right)=p \cdot x-p \cdot \omega_{i} \geqslant 0$ implies $p \cdot x \geqslant p \cdot \omega_{i}$. This means that $\left(x_{1}, \ldots, x_{m}\right)$ is a quasiequilibrium for our exchange economy.

To finish the proof, we must establish that $\Gamma \cap \mathbf{G}=\varnothing$. To this end, assume by way of contradiction that $\Gamma \cap \mathbf{G} \neq \varnothing$. Fix $a \in \Gamma \cap \mathbf{G}$ and write

$$
a=\sum_{i=1}^{m} \lambda_{i}\left(z_{i}-\omega_{i}\right)=\alpha w
$$

where $\lambda_{i} \geqslant 0$ and $z_{i} \succcurlyeq_{i} x_{i}$ for each $i, \sum_{i=1}^{m} \lambda_{i}=1, \alpha>0$, and $w \in E$ satisfies $w+v \in \frac{1}{2} V$. For each $i$ pick a sequence $\left\{r_{n}^{i}\right\}$ of strictly positive rational numbers such that $r_{n}^{i} \downarrow_{n} \lambda_{i}$. So there exist positive rational numbers $\frac{n_{i}}{n}$ (with the $n_{i}$ and $n$ positive integers) such that

$$
\left[\frac{1}{\alpha} \sum_{i=1}^{m} \frac{n_{i}}{n}\left(z_{i}-\omega_{i}\right)-w\right]+(w+v) \in \frac{1}{2} V+\frac{1}{2} V=V .
$$

Consequently,

$$
\sum_{i=1}^{m} n_{i} z_{i}-\sum_{i=1}^{m} n_{i} \omega_{i}+\alpha n v \in \alpha n V
$$

Next, put

$$
y=\sum_{i=1}^{m} n_{i} \omega_{i}-\alpha n v \quad \text { and } \quad z=\sum_{i=1}^{m} n_{i} z_{i} \geqslant 0 .
$$

From $z-y=z+\alpha n v-\sum_{i=1}^{m} n_{i} \omega_{i} \leqslant z+\alpha n v$, it follows that

$$
(y-z)^{-}=(z-y)^{+} \leqslant z+\alpha n v=\sum_{i=1}^{m}\left(n_{i} z_{i}+\alpha n v_{i}\right) .
$$

From the Riesz Decomposition Property, there exist positive vectors $w_{i} \in E^{+}(i=$ $1, \ldots, m)$ with $0 \leqslant w_{i} \leqslant n_{i} z_{i}+\alpha n v_{i}$ and $\sum_{i=1}^{m} w_{i}=(y-z)^{-}$. Define

$$
y_{i}=z_{i}+\frac{\alpha n}{n_{i}} v_{i}-\frac{1}{n_{i}} w_{i}=\frac{1}{n_{i}}\left(n_{i} z_{i}+\alpha n v_{i}-w_{i}\right) \geqslant 0,
$$

and note that $y_{i} \succ_{i} z_{i}$ for each $i$. Indeed, if $z_{i}=y_{i}-\frac{\alpha n}{n_{i}} v_{i}+\frac{1}{n_{i}} w_{i} \succcurlyeq_{i} y_{i}$ is true, then the properness implies $\frac{1}{n_{i}} w_{i} \notin \frac{\alpha n}{n_{i}} V$, or $w_{i} \notin \alpha n V$. On the other hand, from 
$0 \leqslant w_{i} \leqslant(y-z)^{-} \leqslant|y-z|=\left|\sum_{i=1}^{m} n_{i}\left(z_{i}-\omega_{i}\right)+\alpha n v\right|$ and $(\star)$, we see that $w_{i} \in \alpha n V$, which contradicts $w_{i} \notin \alpha n V$. So $y_{i} \succ_{i} z_{i}$ for all $i$. Finally, from $y_{i} \succ_{i} z_{i} \succcurlyeq_{i} x_{i}$ and

$$
\begin{aligned}
\sum_{i=1}^{m} n_{i} y_{i} & =\sum_{i=1}^{m} n_{i} z_{i}+\alpha n \sum_{i=1}^{m} v_{i}-\sum_{i=1}^{m} w_{i}=z+\alpha n v-(y-z)^{-} \\
& \leqslant z+\alpha n v+y-z=y+\alpha n v=\sum_{i=1}^{m} n_{i} \omega_{i},
\end{aligned}
$$

it follows that the allocation $\left(x_{1}, \ldots, x_{m}\right)$ can be blocked in some replica economy, which is impossible. Hence, $\Gamma \cap \mathbf{G}=\varnothing$ must be true, as desired.

Now the infinite dimensional analogue of the Debreu-Scarf Core Equivalence Theorem 55 can be formulated as follows.

Theorem 70 In a convex Riesz exchange economy whose preferences are strictly monotone, continuous, and uniformly proper for a consistent locally convex-solid topology and the total endowment is strictly positive, the sets of Walrasian and Edgeworth equilibria coincide.

Proof: Clearly, every Walrasian equilibrium is an Edgeworth equilibrium. On the other hand, any Edgeworth equilibrium in such an exchange economy is (by Theorem 69) a quasiequilibrium which in turn (by Corollary 32) must be a Walrasian equilibrium.

Theorem 71 In the class of convex Riesz exchange economies with strictly positive total endowments, weakly compact Edgeworth boxes, and strictly monotone, continuous, and uniformly proper (for consistent locally convex-solid topologies) preferences, the following statements are equivalent.

1. Every economy has a Walrasian equilibrium.

2. Every economy has a core allocation.

Proof: To see that (2) implies (1), notice that a verbatim repetition of the proof of Theorem 56 guarantees the existence of an allocation that belongs to the core of every replica economy, which, by Theorem 55, must be a Walrasian equilibrium.

It is also interesting to mention the following converse-type result of Theorem 24. If (under the standard hypotheses) in a Riesz commodity exchange economy every Edgeworth equilibrium is supported by a price as a quasiequilibrium, then the price space $X^{\prime}$ is necessarily a Riesz subspace of the order dual $E^{\sim}$ of $E$. For details see [14].

\section{The single sector growth model}

The standard formulation of the single sector optimal growth problem runs something like this. ${ }^{3}$ There is a single commodity which is used as capital, along with labor, to

\footnotetext{
${ }^{3}$ For more details of the standard formulation see, e.g., M. Harris [110, Chapters 2 and 3] or N. Stokey, R. Lucas, and E. Prescott [205, Chapter 5].
} 
produce more output. In the simplest formulation, labor is presumed to be supplied in fixed amounts and capital and consumption are interpreted in per capita terms. Raw output is either consumed or saved as capital until the next period. This process repeats ad infinitum.

Letting $c_{t}$ denote the quantity of output consumed at time $t$ and $k_{t}$ denote the capital stock, where $k_{1}$ is given as a datum, the set of feasible trajectories of the economy is the set of all positive sequences

$$
(c, k)=\left(\left(c_{1}, c_{2}, \ldots\right),\left(k_{1}, k_{2}, \ldots\right)\right)=\left(\left(c_{1}, k_{1}\right),\left(c_{2}, k_{2}\right), \ldots\right)
$$

obeying

$$
k_{t+1}=(1-\delta) k_{t}+f\left(k_{t}\right)-c_{t} \text { and } 0 \leqslant c_{t} \leqslant f\left(k_{t}\right)
$$

for $t=1,2, \ldots$. Here $f:[0, \infty) \rightarrow[0, \infty)$ is the production function and $0<\delta<1$ is the rate of physical depreciation of capital. The production function $f$ is assumed to be continuous, strictly concave, strictly increasing, and to satisfy $f(0)=0, f^{\prime}(0)=\infty$, and $\lim _{k \rightarrow \infty} \frac{f(k)}{k}=0$. The functions $f(k)=k^{p}$, where $0<p<1$, are all acceptable production functions.

Lemma 72 If $f$ is a production function, then for each $m>0$ there exists a unique $k_{m}>0$ such that:

1. $f\left(k_{m}\right)=m k_{m}$;

2. $f(k)>m k$ for each $0<k<k_{m}$; and

3. $f(k)<m k$ for each $k>k_{m}$.

Now we come to the notion of a free disposal trajectory.

Definition 73 The set of free disposal feasible trajectories with respect to a production function $f$, some $k_{1}>0$, and some $0<\delta<1$ is the set $Y \subset \ell_{\infty}^{+} \times \ell_{\infty}^{+}$defined by

$$
Y=\left\{(c, k) \in\left(\ell_{\infty}^{+}\right)^{2}: \begin{array}{l}
0 \leqslant k_{t+1} \leqslant(1-\delta) k_{t}+f\left(k_{t}\right)-c_{t} \\
0 \leqslant c_{t} \leqslant f\left(k_{t}\right)
\end{array}, t=1,2, \ldots\right\} .
$$

Let 1 denote the constant sequence one in $\ell_{\infty} \times \ell_{\infty}$, i.e., $\mathbf{1}=((1,1),(1,1), \ldots)$.

Lemma 74 Every feasible free disposal set of trajectories $Y$ is a convex norm bounded subset of $\ell_{\infty}^{+} \times \ell_{\infty}^{+}$and satisfies $0 \in Y$ and $\eta \mathbf{1} \in Y$ for some $\eta>0$.

Proof: Let $Y$ be a free disposal feasible set of trajectories with respect to a production function $f$ and some $k_{1}>0$. Clearly, $Y$ is a convex set. Next, we establish that $Y$ is norm bounded. To this end, let $(c, k) \in Y$. Since $\lim _{k \rightarrow \infty} \frac{f(k)}{k}=0$, there exists $n$ such that $f(k)<\delta k$ for each $k \geqslant n$. Put $M=\sup _{k \in[0, \infty)}[f(k)-\delta k]=\max _{k \in[0, n]}[f(k)-\delta k]$ 
and let $b=\max \left\{n+M, k_{1}\right\}<\infty$. We claim that $k_{t} \leqslant b$ for each $t$. The proof is by induction. For $t=1$, the inequality is obvious. Now for the induction step, assume that $k_{t} \leqslant b$. If $k_{t}>n$, then

$$
k_{t+1}=(1-\delta) k_{t}+f\left(k_{t}\right)-c_{t}=k_{t}+\left[f\left(k_{t}\right)-\delta k_{t}\right]-c_{t}<k_{t} \leqslant b .
$$

If $k_{t} \leqslant n$, then

$$
k_{t+1}=k_{t}+\left[f\left(k_{t}\right)-\delta k_{t}\right]-c_{t} \leqslant n+M \leqslant b,
$$

and the proof of our claim is complete. To see that the sequence $\left\{c_{t}\right\}$ is uniformly bounded, note that

$$
0 \leqslant c_{t} \leqslant f\left(k_{t}\right) \leqslant \max _{0 \leqslant k \leqslant b} f(k)
$$

for each $t$. Finally, note that $0 \in Y$ and if $f(\eta)=(1+\delta) \eta$, then $\eta \mathbf{1} \in Y$.

It is also easy to see that since $Y$ is defined pointwise by inequalities among continuous functions, that $Y$ is pointwise closed. In particular, $Y$ is closed for the $\sigma\left(\ell_{\infty} \times \ell_{\infty}, \ell_{1} \times \ell_{1}\right)$ topology. This leads to the next lemma.

Lemma 75 Every set of feasible free disposal trajectories $Y$ is $\sigma\left(\ell_{\infty} \times \ell_{\infty}, \ell_{1} \times \ell_{1}\right)$ compact.

Proof: Recall that the norm dual of $\ell_{1}$ is the Banach lattice $\ell_{\infty}$. Since $Y$ is norm bounded and $\sigma\left(\ell_{\infty} \times \ell_{\infty}, \ell_{1} \times \ell_{1}\right)$-closed, Alaoglu's Theorem [2, Theorem 4.79, p.158] shows that $Y$ is $\sigma\left(\ell_{\infty} \times \ell_{\infty}, \ell_{1} \times \ell_{1}\right)$-compact.

The purpose of the optimal growth problem is to find the feasible plan $(c, k)$ maximizing the objective function

$$
U(c, k)=\sum_{t=1}^{\infty} \beta^{t} u\left(c_{t}\right),
$$

where $u:[0, \infty) \rightarrow \mathbb{R}$ is continuous, concave, strictly increasing, and normalized so that $u(0)=0$ and $0<\beta<1$ is a constant called the discount factor. Note that in case $\left\{x_{t}\right\}$ is bounded so is $\left\{u\left(x_{t}\right)\right\}$, so $\sum_{t=1}^{\infty} \beta^{t} u\left(x_{t}\right)$ is a real number. Therefore, $U: \ell_{\infty}^{+} \times \ell_{\infty}^{+} \rightarrow \mathbb{R}$ is a well defined function. The objective function $U$ may be interpreted either as the social welfare function of a central planner or as the utility function of a representative agent in the economy who has complete control of his/her own consumption and production. Since $Y$ is $\sigma\left(\ell_{\infty} \times \ell_{\infty}, \ell_{1} \times \ell_{1}\right)$-compact an optimal plan is guaranteed to exist if $U$ is $\sigma\left(\ell_{\infty} \times \ell_{\infty}, \ell_{1} \times \ell_{1}\right)$-upper semicontinuous; see Theorem [2, Theorem 2.43]. Indeed, as we shall see, $U$ is $\sigma\left(\ell_{\infty} \times \ell_{\infty}, \ell_{1} \times \ell_{1}\right)$-continuous on the free disposal feasible trajectory sets. Any free disposal trajectory that maximizes $U$ over a free disposal feasible trajectory set $Y$ is known as an optimal plan.

Lemma 76 If $Y$ is a free disposal feasible trajectory set, then every optimal plan $(c, k)$ is a feasible trajectory, i.e.,

$$
k_{t+1}=(1-\delta) k_{t}+f\left(k_{t}\right)-c_{t} \quad \text { and } \quad 0 \leqslant c_{t} \leqslant f\left(k_{t}\right)
$$

for each $t$. 
We next note the following properties of the utility function.

Lemma 77 For a concave nondecreasing function $u:[0, \infty) \rightarrow[0, \infty)$ satisfying $u(0)=0$ the following statements hold.

1. If $0<x \leqslant y$, then $\frac{u(y)}{y} \leqslant \frac{u(x)}{x}$.

2. If $x>0$ and $y \geqslant 0$, then

$$
|u(x)-u(y)| \leqslant \frac{u(x)}{x}|x-y|
$$

3. If $0 \leqslant x \leqslant z \leqslant y$ and $z>0$, then

$$
|u(x)-u(y)| \leqslant \frac{u(z)}{z}|x-y| .
$$

Let $u:[0, \infty) \rightarrow[0, \infty)$ be a nondecreasing utility function. Then the discounted utility of $u$ with respect to $0<\beta<1$ is the function $U: \ell_{\infty}^{+} \rightarrow[0, \infty)$.defined by

$$
U(x)=\sum_{t=1}^{\infty} \beta^{t} u\left(x_{t}\right)
$$

Theorem 78 Let $u:[0, \infty) \rightarrow \mathbb{R}$ be a nondecreasing concave function satisfying $u(0)=0$ and let $U$ be the discounted utility of $u$ with respect to some $0<\beta<1$. Then:

1. $U$ is nondecreasing, concave, and $|\sigma|\left(\ell_{\infty}, \ell_{1}\right)$-continuous;

2. $U$ is $\sigma\left(\ell_{\infty}, \ell_{1}\right)$-continuous on the norm bounded subsets of $\ell_{\infty}^{+}$; and

3. $U$ is norm continuous.

Proof: (1) To show that $U$ is $|\sigma|\left(\ell_{\infty}, \ell_{1}\right)$-continuous, let $x^{\alpha} \stackrel{|\sigma|\left(\ell_{\infty}, \ell_{1}\right)}{\longrightarrow} x$ in $\ell_{\infty}^{+}$. We wish to show that $\left|U\left(x^{\alpha}\right)-U(x)\right| \underset{\alpha}{\rightarrow}$. To this end, let $\varepsilon>0$.

Choose $\eta>0$ such that $\sum_{t=1}^{\infty} \beta^{t} u(\eta)<\varepsilon$ and let $B_{\eta}=\left\{t: x_{t}<\eta\right\}$. From $\sum_{t=1}^{\infty} \beta^{t} u\left(x_{t}\right)<\infty$, we see that $\left(\beta^{1} u\left(x_{1}\right), \beta^{2} u\left(x_{2}\right), \beta^{3} u\left(x_{3}\right), \ldots\right) \in \ell_{1}$. So there exists (since $\left.x^{a} \stackrel{|\sigma|\left(\ell_{\infty}, \ell_{1}\right)}{\alpha} 0\right)$ some $\alpha_{0}$ such that

$$
\sum_{t=1}^{\infty} \beta^{t} \frac{u\left(x_{t}\right)}{\eta}\left|x_{t}^{\alpha}-x_{t}\right|<\varepsilon \quad \text { and } \quad \sum_{t=1}^{\infty} \beta^{t} \frac{u(\eta)}{\eta}\left|x_{t}^{\alpha}-x_{t}\right|<\varepsilon
$$

for all $\alpha \geqslant \alpha_{0}$.

Now let $t \in B_{\eta}$. If $x_{t}^{\alpha}>\eta$, then $\left|u\left(x_{t}^{\alpha}\right)-u\left(x_{t}\right)\right| \leqslant \frac{u(\eta)}{\eta}\left|x_{t}^{\alpha}-x_{t}\right|$ (Lemma 77), and if $x_{t}^{\alpha}<\eta$, then $\left|u\left(x_{t}^{\alpha}\right)-u\left(x_{t}\right)\right| \leqslant 2 u(\eta)$. Thus,

$$
\sum_{t \in B_{\eta}} \beta^{t}\left|u\left(x_{t}^{\alpha}\right)-u\left(x_{t}\right)\right| \leqslant \sum_{t \in B_{\eta}} \beta^{t} \frac{u(\eta)}{\eta}\left|x_{t}^{\alpha}-x_{t}\right|+2 \sum_{t \in B_{\eta}} \beta^{t} u(\eta)<\varepsilon+2 \varepsilon=3 \varepsilon .
$$


Finally, using Lemma 77 once more, we see that

$$
\begin{aligned}
\left|U\left(x^{\alpha}\right)-U(x)\right| & =\left|\sum_{t=1}^{\infty} \beta^{t} u\left(x_{t}^{\alpha}\right)-\sum_{t=1}^{\infty} \beta^{t} u\left(x_{t}\right)\right| \leqslant \sum_{t=1}^{\infty} \beta^{t}\left|u\left(x_{t}^{\alpha}\right)-u\left(x_{t}\right)\right| \\
& =\sum_{t \in B_{\eta}} \beta^{t}\left|u\left(x_{t}^{\alpha}\right)-u\left(x_{t}\right)\right|+\sum_{t \in B_{\eta}^{c}} \beta^{t}\left|u\left(x_{t}^{\alpha}\right)-u\left(x_{t}\right)\right| \\
& \leqslant \sum_{t \in B_{\eta}} \beta^{t}\left|u\left(x_{t}^{\alpha}\right)-u\left(x_{t}\right)\right|+\sum_{t \in B_{\eta}^{c}} \beta^{t} \frac{u\left(x_{t}\right)}{x_{t}}\left|x_{t}^{\alpha}-x_{t}\right| \\
& \leqslant \sum_{t \in B_{\eta}} \beta^{t}\left|u\left(x_{t}^{\alpha}\right)-u\left(x_{t}\right)\right|+\sum_{t \in B_{\eta}^{c}} \beta^{t} \frac{u\left(x_{t}\right)}{\eta}\left|x_{t}^{\alpha}-x_{t}\right| \\
& <3 \varepsilon+\varepsilon=4 \varepsilon .
\end{aligned}
$$

for all $\alpha \geqslant \alpha_{0}$, as desired.

(2) Assume $x^{\alpha} \stackrel{\sigma\left(\ell_{\infty}, \ell_{1}\right)}{\longrightarrow} x$ and that $\|x\|_{\infty} \leqslant b<\infty$ and $\left\|x^{\alpha}\right\|_{\infty} \leqslant b$ for each $\alpha$. Fix $\varepsilon>0$ and then choose some $n$ such that $2 \sum_{t=n+1}^{\infty} \beta^{t} u(b)<\varepsilon$. Thus,

$$
\left|U\left(x^{\alpha}\right)-U(x)\right| \leqslant \sum_{t=1}^{n} \beta^{t}\left|u\left(x_{t}^{\alpha}\right)-u\left(x_{t}\right)\right|+\varepsilon .
$$

Taking into account that $x^{\alpha} \stackrel{\sigma\left(\ell_{\infty}, \ell_{1}\right)}{\longrightarrow} x$ implies $x_{t}^{\alpha} \underset{\alpha}{\rightarrow} x_{t}$ for each $t$, the preceding inequality yields $\lim \sup _{\alpha}\left|U\left(x^{\alpha}\right)-U(x)\right| \leqslant \varepsilon$ for each $\varepsilon>0$. Therefore, $U\left(x^{\alpha}\right) \underset{\alpha}{\rightarrow} U(x)$.

(3) This follows from the fact that every norm convergent sequence is norm bounded and weakly convergent.

Before proceeding further, let us introduce the notion of a single agent growth economy. The single agent growth model economy has the following characteristics.

1. The commodity-price duality $\left\langle E, E^{\prime}\right\rangle$ is the symmetric Riesz pair $\left\langle\ell_{\infty} \times \ell_{\infty}, \ell_{1} \times \ell_{1}\right\rangle$.

2. The production possibility set is a set $Y \subset E^{+}$which is convex, weakly compact, and satisfies $0 \in Y$ and $\eta \mathbf{1} \in Y$ for some $\eta>0$.

3. There is a single agent who in his/her capacity as the manager of production chooses output to maximize the value of output treating prices parametrically. As a consumer the agent maximizes a preference relation $\succcurlyeq$ defined on $E^{+}$subject to a budget constraint, treating prices and income as parameters. The preference $\succcurlyeq$ is assumed to be strictly monotone, convex, and $\tau$-continuous for a consistent locally convex linear topology $\tau$ on $E$.

We have already seen that the standard formulation of the single sector growth model with discounted utility fits this framework. 
Definition $79 A$ competitive equilibrium for a single agent growth economy is a pair $(x, p)$ in $E \times E^{\prime}$ such that:

1. $x \in Y$ and $p \neq 0$;

2. the bundle $x$ maximizes the value of output at prices $p$, i.e., $p \cdot x \geqslant p \cdot y$ for all $y \in Y$; and

3. the bundle $x$ also maximizes the preference $\succcurlyeq$ subject to the budget constraint. That is, letting $B=\left\{z \in E^{+}: p \cdot z \leqslant p \cdot x\right\}$, then $x \succcurlyeq z$ for all $z \in B$.

The next result is closely related to results obtained by R. E. Lucas and E. Prescott [137, 179] and A. Araujo and P. Monteiro [24].

Theorem 80 Every single agent growth economy has a competitive equilibrium.

Proof: Since $Y$ is $\sigma\left(E, E^{\prime}\right)$-compact and $\succcurlyeq$ is $\tau$-continuous there is a $\succcurlyeq$-greatest element $x$ of $Y$; see [8, Theorem 1.2.2, p. 14].

By Theorem 19, we know that $\succcurlyeq$ is norm proper. Thus, $\succcurlyeq$ is $(v, W)$-uniformly proper for some norm open ball $W$ centered at the origin. Therefore, $v$ is a desirable bundle. Consider the cone

$$
\Gamma=\{\alpha(-v+w): \alpha>0 \text { and } w \in W\} .
$$

Since $-v \in \Gamma$ and $-v+W \subseteq \Gamma$, we see that $\Gamma$ has a (norm) nonempty interior. Now if $F$ is the preferred set of $x$, i.e., if $F=\{z: z \succcurlyeq x\}$, then we claim that $(F-Y) \cap \Gamma=\varnothing$.

To see this, assume by way of contradiction that $(F-Y) \cap \Gamma \neq \varnothing$. Then there exist $\alpha>0, z \in F, y \in Y$, and $w \in W$ satisfying

$$
z-y=\alpha(-v+w)
$$

Then $z=y-\alpha v+\alpha w, z \succcurlyeq x$, and $x \succcurlyeq y$ since $x$ is maximal in $Y$. Thus $y-\alpha v+\alpha w=z \succcurlyeq x$ and $x \succcurlyeq y$. Therefore, we have $y-\alpha v+\alpha w \succcurlyeq y$, and by properness $\alpha w \notin \alpha W$, a contradiction. Thus, $(F-Y) \cap \Gamma=\varnothing$, as claimed.

Next, by the Separation Theorem [2, Theorem 4.46, p. 143] there exist a non-zero price $q$ in the norm dual of $E$ (which may not lie in $E^{\prime}$ ) and some constant $c$ such that

$$
q \cdot(z-y) \geqslant c \geqslant q \cdot g
$$

for all $z-y \in F-Y$ and all $g \in \Gamma$. Since $\Gamma$ is a cone, we see that $c \geqslant 0$. Also $x \in Y$, so if $z \succcurlyeq x$ then $z-x \in F-Y$, so $q \cdot z \geqslant q \cdot x$. Moreover, $x-y \in F-Y$, so $q \cdot x \geqslant q \cdot y$ for all $y \in Y$.

Since for each $z \geqslant 0$, we have $(x+z)-x \in F-Y$, we see that $q \cdot(x+z) \geqslant q \cdot x$, or $q \cdot z \geqslant 0$. Therefore, $q>0$. In particular, $q \cdot \mathbf{1}>0$. Since $\eta \mathbf{1} \in Y$, this shows that $q \cdot x>0$. 
By the Yosida-Hewitt representation [2, p. 250], we can write $q=p+s$, where $0 \leqslant p \in \ell_{1} \times \ell_{1}, s \geqslant 0$ and $s \cdot z=0$ for all $z \in c_{0} \times c_{0}$. To finish the proof, we show that $(x, p)$ is an equilibrium (cf. [179]).

Pick a sequence $\left\{x_{n}\right\} \in c_{0} \times c_{0}$ such that $0 \leqslant x_{n} \uparrow x$ and note that $x_{n} \stackrel{\tau}{\rightarrow} x$. Fix $\varepsilon>0$. From $x+\varepsilon v \succ x$, we see that there exists some $n_{0}$ such that $x_{n}+\varepsilon v \succ x$ for all $n \geqslant n_{0}$. So $q \cdot\left(x_{n}+\varepsilon v\right)=q \cdot x_{n}+\varepsilon q \cdot v \geqslant q \cdot x$ for all $n \geqslant n_{0}$. Thus, $0 \leqslant q \cdot\left(x-x_{n}\right) \leqslant \varepsilon q \cdot v$ for all $n \geqslant n_{0}$, which means that $\lim q \cdot x_{n}=q \cdot x$. Since $p$ is also $\sigma\left(E, E^{\prime}\right)$-continuous and $q \cdot x_{n}=p \cdot x_{n}+s \cdot x_{n}=p \cdot x_{n}$, we see that $p \cdot x=q \cdot x$. In particular, note that $p \neq 0$.

Now we are ready to show that $(x, p)$ is an equilibrium. Let $y \in Y$. Then $y \geqslant 0$ and $q \geqslant p$, so $p \cdot x=q \cdot x \geqslant q \cdot y \geqslant p \cdot y$. Next, let $z \succ x$. It easily follows that there exists some $0 \leqslant z_{0} \leqslant z$ such that $z_{0} \succ x$ and $z_{0} \in c_{0} \times c_{0}$. Choose some $0<\delta<1$ such that $\delta z_{0} \succ x$. Then $p \cdot\left(\delta z_{0}\right)=(p+s) \cdot\left(\delta z_{0}\right)=q \cdot\left(\delta z_{0}\right) \geqslant q \cdot x=p \cdot x>0$. Since $p \geqslant 0$ and $z \geqslant z_{0}$, we get $p \cdot z \geqslant \delta p \cdot z \geqslant \delta p \cdot z_{0} \geqslant p \cdot x>0$, which shows that $p \cdot z>p \cdot x$. In other words, $x$ is the maximal element in the budget set, and the proof is finished.

\section{Extended Bibliography}

[1] Aliprantis, C. D. forthcoming, 1996. Problems in equilibrium theory. Springer-Verlag.

[2] Aliprantis, C. D. and K. C. Border. 1994. Infinite dimensional analysis. Berlin: Springer-Verlag.

[3] Aliprantis, C. D. and D. J. Brown. 1983. Equilibria in markets with a Riesz space of commodities. Journal of Mathematical Economics 11:189-207.

[4] Aliprantis, C. D., D. J. Brown, and O. Burkinshaw. 1987. An economy with infinite dimensional commodity space and empty core. Economic Letters 23:1-4.

[5] Aliprantis, C. D., D. J. Brown, and O. Burkinshaw. 1987. Edgeworth equilibria. Econometrica 55(5):1109-1137.

[6] Aliprantis, C. D., D. J. Brown, and O. Burkinshaw. 1987. Edgeworth equilibria in production economies. Journal of Economic Theory 43:252-291.

[7] Aliprantis, C. D., D. J. Brown, and O. Burkinshaw. 1989. Equilibria in exchange economies with a countable number of agents. Journal of Mathematical Analysis and Applications 142:250-299.

[8] Aliprantis, C. D., D. J. Brown, and O. Burkinshaw. 1990. Existence and optimality of competitive equilibria. New York: Springer-Verlag.

[9] Aliprantis, C. D., D. J. Brown, and O. Burkinshaw. 1990. Valuation and optimality in the overlapping generations model. International Economic Review 31:275-288.

[10] Aliprantis, C. D. and O. Burkinshaw. 1978. Locally solid Riesz spaces. Number 76 in Pure and Applied Mathematics. New York: Academic Press.

[11] Aliprantis, C. D. and O. Burkinshaw. 1985. Positive operators. Number 119 in Pure and Applied Mathematics. New York: Academic Press.

[12] Aliprantis, C. D. and O. Burkinshaw. 1988. The fundamental theorems of welfare economies without proper preferences. Journal of Mathematical Economics 17(1):41-54.

[13] Aliprantis, C. D. and O. Burkinshaw. 1990. An overlapping generations model core equivalence theorem. Journal of Economic Theory 50(2):362-380. 
[14] Aliprantis, C. D. and O. Burkinshaw. 1991. When is the core equivalence theorem valid? Economic Theory 1:169-18.

[15] Aliprantis, C. D., O. Burkinshaw, and N. J. Rothman, eds. 1985. Advances in equilibrium theory. Number 244 in Lecture Notes in Economics and Mathematical Systems. Heidelberg \& New York: Springer-Verlag.

[16] Allingham, M. 1975. General equilibrium. New York: Halsted Press.

[17] Anderson, R. M. 1978. An elementary core equivalence theorem. Econometrica 46:1483-1487.

[18] Anderson, R. M. 1981. Core theory with strongly convex preferences. Econometrica 49:1457-1468.

[19] Anderson, R. M. 1986. Notions of core convergence. In [113], pages 25-46.

[20] Anderson, R. M. and W. R. Zame. 1994. Core convergence from A to Z. Working paper, University of California (Berkeley).

[21] Araujo, A. 1985. Lack of Pareto optimality in economies with infinitely many commodities: The need for impatience. Econometrica 53:455-461.

[22] Araujo, A. 1985. Regular economies and sets of measure zero in Banach spaces. Journal of Mathematical Economics 14(1):61-66.

[23] Araujo, A. and P. Monteiro. 1989. Equilibrium without uniform conditions. Journal of Economic Theory 48:416-427.

[24] Araujo, A. and P. Monteiro. 1994. The general existence of extended price equilibria with infinitely many commodities. Journal of Economic Theory 63:408-416.

[25] Arrow, K. J. 1951. An extension of the basic theorems of classical welfare economics. In J. Neyman, ed., Proceedings of the Second Berkeley Symposium on Mathematical Statistics and Probability II, Part I, pages 507-532. University of California Press, Berkeley.

[26] Arrow, K. J. 1969. Tullock and an existence theorem. Public Choice 6:105-111.

[27] Arrow, K. J. and G. Debreu. 1954. Existence of an equilibrium for a competitive economy. Econometrica 22:265-290.

[28] Arrow, K. J. and F. Hahn. 1971. General competitive analysis. San Francisco: Holden-Day.

[29] Aubin, J.-P. 1979. Mathematical methods of game and economic theory. Number 7 in Studies in mathematics and its applications. New York: North Holland.

[30] Aumann, R. J. 1966. Existence of competitive equilibria in markets with a continuum of traders. Econometrica 34:1-17.

[31] Back, K. 1988. Structure of consumption sets and existence of equilibria in infinite-dimensional spaces. Journal of Mathematical Economics 17(1):89-99.

[32] Balasko, Y. 1988. Foundations of the theory of general economic equilibrium. Orlando, Fla.: Academic Press.

[33] Balasko, Y., D. Cass, and K. Shell. 1980. Existence of competitive equilibrium in a general overlapping-generations model. Journal of Economic Theory 23:307-322.

[34] Balasko, Y. and K. Shell. '1980. The overlapping generations model. I. The case of pure exchange without money. Journal of Economic Theory 23:281-306.

[35] Balasko, Y. and K. Shell. 1981. The overlapping generations model. II. The case of pure exchange with money. Journal of Economic Theory 24:112-142.

[36] Balasko, Y. and K. Shell. 1981. The overlapping-generations model, III: The case of log-linear utility functions. Journal of Economic Theory 24:143-152. 
[37] Baumol, W. and S. M. Goldfeld, eds. 1968. Precursors in mathematical economics. Number 19 in Series of reprints of scarce works on political economy. London: London School of Economics and Political Science.

[38] Becker, R. A. 1991. Fundamental theorems of welfare economics in infinite dimensional commodity spaces. In [129], pages 125-175.

[39] Becker, R. A., H. Bercovici, and C. Foias. 1993. Weak Pareto optimality and the approximate support property. Journal of Mathematical Economics 22:61-71.

[40] Becker, R. A. and I. Boyd, J. H. forthcoming. Capital theory, equilibrium analysis and recursive utility. Basil Blackwell.

[41] Becker, R. A., I. Boyd, J. H., and C. Foias. 1991. The existence of Ramsey equilibrium. Econometrica 59:441-460.

[42] Becker, R. A., J. H. Boyd, III, and B. Y. Sung. 1988. Recursive utility and optimal capital accumulation I: Existence. Journal of Economic Theory 47:76-100.

[43] Becker, R. A. and C. Foias. 1987. A characterization of Ramsey equilibrium. Journal of Economic Theory 41:173-184.

[44] Bergstrom, T. C. 1976. How to discard 'free disposability' - at no cost. Journal of Mathematical Economics 3:131-134.

[45] Berliant, M. 1985. An equilibrium existence result for an economy with land. Journal of Mathematical Economics 14(1):53-56.

[46] Bewley, T. F. 1969. A theorem on the existence of competitive equilibria in a market with a finite number of agents whose commodity space is $l_{\infty}$. Discussion paper, CORE, Université de Louvain.

[47] Bewley, T. F. 1972. Existence of equilibria in economies with infinitely many commodities. Journal of Economic Theory 4:514-540.

[48] Bewley, T. F. 1981. Stationary equilibrium. Journal of Economic Theory 24:265-295.

[49] Bewley, T. F. 1982. An integration of equilibrium theory and turnpike theory. Journal of Mathematical Economics 10(2/3):233-267.

[50] Bidard, C. 1989. Equilibrium with a qualitative Walras' law. Journal of Economic Theory 47:203205.

[51] Boehm, V. 1974. The core of an economy with production. Review of Economic Studies 41:429436.

[52] Bojan, P. 1974. A generalization of theorems on the existence of competitive equilibrium to the case of infinitely many commodities. Mathematica Balkanica 4:491-494.

[53] Bondareva, O. N. 1962. Kernel theory in n-person games. Vestnik Leningrad University. Mathematics 17:141-142.

[54] Border, K. C. 1983. On equilibria of excess demand correspondences. Social Science Working Paper 460, California Institute of Technology.

[55] Border, K. C. 1984. A core existence theorem without ordered preferences. Econometrica 52(6):1537-1542.

[56] Border, K. C. 1985. Fixed point theorems with applications to economics and game theory. New York: Cambridge University Press.

[57] Boyd, III, J. H. 1990. Recursive utility and the Ramsey problem. Journal of Economic Theory 50(2):346-361.

[58] Boyd, III, J. H. and L. McKenzie. 1993. The existence of competitive equilibrium over an infinite horizon with production and general consumption sets. International Economic Review 34:1-20. 
[59] Brown, D. J. 1991. Equilibrium analysis with non-convex technologies. In [114], chapter 36, pages 1963-1995.

[60] Brown, D. J. and L. M. Lewis. 1981. Myopic economic agents. Econometrica 49:359-368.

[61] Brown, D. J. and J. Werner. 1995. Arbitrage and existence of equilibrium in infinite asset markets. Review of Economic Studies 62:101-114.

[62] Burke, J. 1988. On the existence of price equilibria in dynamic economies. Journal of Economic Theory 44:281-300.

[63] Cass, D., M. Okuno, and I. Zilcha. 1979. The role of money in supporting the Pareto optimality of competitive equilibrium in consumption-loan type models. Journal of Economic Theory 20:41-80.

[64] Cass, D. and M. E. Yaari. 1966. A re-examination of the pure-consumption loans model. Journal of Political Economy 74:353-367.

[65] Chae, S. 1987. Short-run core equivalence in an overlapping generations model. Journal of Economic Theory 24:112-142.

[66] Cheng, H. 1990. Supply and demand analysis in infinite dimensional economies. Manuscript.

[67] Cherif, I., M. Deghdak, and M. Florenzano. 1994. Existence of equilibria in the overlapping generations model. Economic Theory 4:217-234.

[68] Chichilnisky, G. 1981. Existence and optimality of optimal growth paths including models with non convexities in utilities and technologies. Review of Economic Studies 48:51-61.

[69] Chichilnisky, G. 1981. Existence of optimal savings policies with imperfect information and non convexities. Journal of Mathematical Economics 8:1-14.

[70] Chichilnisky, G. 1993. The cone condition, properness, and extremely desirable commodities. Economic Theory 3:177-182.

[71] Chichilnisky, G. and G. M. Heal. 1993. Competitive equilibrium in Sobolev spaces without bounds on short sales. Journal of Economic Theory 59:364-384.

[72] Chichilnisky, G. and P. J. Kalman. 1980. Application of functional analysis to models of efficent allocation of economic resources. Journal of Optimization Theory and Applications 30:19-32.

[73] Chipman, J. S. 1977. A renewal model of economic growth. Econometrica 45:295-316.

[74] Chipman, J. S. and J. C. Moore. 1971. The compensation principle in welfare economics. In A. M. Zarley, ed., Papers in Quantitative Economics, volume 2, pages 1-77. University of Kansas Press, Lawrence, Kansas.

[75] Cornet, B., ed. 1988. Special issue: General equilibrium theory and increasing returns. Journal of Mathematical Economics 17(2/3).

[76] Court, L. M. 1941. Entrepreneurial and consumer demand theories for commodity spectra. Econometrica 9:135-162.

[77] Debreu, G. 1951. The coefficient of resource utilization. Econometrica 19:257-273.

[78] Debreu, G. 1952. A social equilibrium existence theorem. Proceedings of the National Academy of Sciences, U.S.A. 38:386-393.

[79] Debreu, G. 1954. Representation of a preference ordering by a numerical function. In R. M. Thrall, C. H. Coombs, and R. L. Davis, eds., Decision Processes, chapter 11, pages 159-166. John Wiley and Sons, New York.

[80] Debreu, G. 1954. Valuation equilibrium and Pareto optimum. Proceedings of the National Academy of Sciences, U.S.A. 40:588-592.

[81] Debreu, G. 1956. Market equilibrium. Proceedings of the National Academy of Sciences, U.S.A. $42: 876-878$. 
[82] Debreu, G. 1959. Theory of value. New Haven: Yale University Press.

[83] Debreu, G. 1962. New concepts and techniques for equilibrium analysis. International Economic Review 3:257-273.

[84] Debreu, G. 1970. Economies with a finite set of equilibria. Econometrica 38:387-392.

[85] Debreu, G. 1974. Excess demand functions. Journal of Mathematical Economics 1:15-21.

[86] Debreu, G. 1982. Existence of competitive equilibrium. In K. J. Arrow and M. D. Intriligator, eds., Handbook of Mathematical Economics, volume 2, chapter 15, pages 697-743. North Holland, Amsterdam.

[87] Debreu, G. and H. E. Scarf. 1963. A limit theorem on the core of an economy. International Economic Review 4:235-246.

[88] Dierker, E. 1974. Topological methods in walrasian economics. Number 92 in Lecture Notes in Economics and Mathematical Systems. Berlin, Heidelberg, \& New York: Springer-Verlag.

[89] Duffie, D. 1986. Competitive equilibria in general choice spaces. Journal of Mathematical Economics 15(1):1-23.

[90] Duffie, D. 1987. Stochastic equilibria with incomplete financial markets. Journal of Economic Theory 41(2):405-416.

[91] Duffie, D. and C. F. Huang. 1985. Implementing Arrow-Debreu equilibria by continuous trading of few long-lived securities. Econometrica 53:1337-1356.

[92] Duffie, D. and W. Zame. 1989. The consumption-based capital asset pricing model. Econometrica $57(6): 1279-1297$.

[93] Edgeworth, F. Y. 1881. Mathematical psychics. London: Kegan Paul.

[94] Esteban, J. 1986. A characterization of the core in overlapping-generations economies. Journal of Economic Theory 39:439-456.

[95] Flåm, Sjur D. and Wets, Roger J-B. 1987. Existence results and finite horizon approximates for infinite horizon optimization problems. Econometrica 55(5):1187-1209.

[96] Florenzano, M. 1982. The Gale-Nikaidô-Debreu lemma and the existence of transitive equilibrium with or without the free disposal assumption. Journal of Mathematical Economics 9:113-134.

[97] Florenzano, M. 1983. On the existence of equilibria in economies with an infinite dimensional commodity space. Journal of Mathematical Economics 12(3):207-219.

[98] Florenzano, M. 1989. On the nonemptiness of the core of a coalitional production economy without ordered preferences. Journal of Mathematical Analysis and Applications 141:484-490.

[99] Florenzano, M. 1990. Edgeworth equilibria, fuzzy core, and equilibria of a production economy without ordered preferences. Journal of Mathematical Analysis and Applications 153(1):18-36.

[100] Florenzano, M. 1990. Markets with countably many periods. Discussion Paper 9019, CEPREMAP.

[101] Florenzano, M. 1991. Quasiequilibria in abstract economies: Application to the overlapping generations model. Discussion Paper 9117, CEPREMAP.

[102] Gale, D. 1955. The law of supply and demand Mathematica Scandinavica 3:155-169.

[103] Gale, D. 1973. Pure exchange equilibrium of dynamic economic models. Journal of Economic Theory 6:12-36.

[104] Gale, D. and A. Mas-Colell. 1975. An equilibrium existence theorem for a general model without ordered preferences. Journal of Mathematical Economics 2:9-15.

[105] Gale, D. and A. Mas-Colell. 1979. Corrections to an equilibrium existence theorem for a general model without ordered preferences. Journal of Mathematical Economics 6:297-298. 
[106] Geanakoplos, J. The overlapping generations model of general equilibrium. In The New Palgrave, pages 767-779. Macmillan, New York \& London.

[107] Geanakoplos, J. and H. Polemarchakis. 1982. Intertemporally separable, overlapping-generations economies. Journal of Economic Theory 34:207-215.

[108] Geanakoplos, J. and H. Polemarchakis. 1991. Overlapping generations, chapter 35, pages 18991961. Volume 4 of [114].

[109] Gilles, C. 1989. Charges as equilibrium prices and asset bubbles. Journal of Mathematical Economics 18(2):155-167.

[110] Harris, M. 1987. Dynamic economic analysis. New York: Oxford University Press.

[111] Hildenbrand, W. 1974. Core and equilibria of a large economy. Princeton: Princeton University Press.

[112] Hildenbrand, W. and A. P. Kirman. 1976. Introduction to equilibrium analysis. Number 6 in Advanced Textbooks in Economics. Amsterdam: North Holland.

[113] Hildenbrand, W. and A. Mas-Colell, eds. 1986. Contributions to mathematical economics. New York: North Holland.

[114] Hildenbrand, W. and H. Sonnenschein, eds. 1991. Handbook of mathematical economics, volume 4. New York: North Holland.

[115] Hurwicz, L. 1958. Programming in linear spaces. In K. J. Arrow, L. Hurwicz, and H. Uzawa, eds., Studies in Linear and Non-linear Programming, number 2 in Stanford Mathematical Studies in the Social Sciences, chapter 4, pages 38-102. Stanford University Press, Stanford, California.

[116] Ichiishi, T. 1981. A social coalitional equilibrium existence theorem. Econometrica 49:369-377.

[117] Jones, L. E. 1983. Existence of equilibria with infinitely many consumers and infinitely many commodities: A theorem based on models of commodity differentiation. Journal of Mathematical Economics 12(2):119-138.

[118] Jones, L. E. 1984. A competitive model of commodity differentiation. Econometrica 52:507-530.

[119] Jones, L. E. 1986. Special problems arising in the study of economies with infinitely many commodities. In H. F. Sonnenschein, ed., Models of Economic Dynamics, number 264 in Lecture Notes in Economics and Mathematical Systems, pages 184-205. Springer-Verlag, Berlin.

[120] Jones, L. E. 1987. The efficiency of monopolistically competitive equilibria in large economies: Commodity differentiation with gross substitutes. Journal of Economic Theory 41(2):356-391.

[121] Jones, L. E. 1987. Existence of equilibria with infinitely many commodities: Banach lattices reconsidered. Journal of Mathematical Economics 16(2):89-104.

[122] Jones, L. E. 1992. Equilibrium in competitive infinite dimensional settings. In J.-J. Laffont, ed., Advances in Economic Theory, Sixth World Congress, volume 2, chapter 7, pages 413-443. Cambridge University Press.

[123] Kehoe, T. J. and D. K. Levine. 1984. Intertemporal separability in overlapping-generations models. Journal of Economic Theory 34:216-226.

[124] Kehoe, T. J. and D. K. Levine. 1984. Regularity in overlapping generations exchange economies. Journal of Mathematical Economics 13:69-93.

[125] Khan, M. A. 1984. A remark on the existence of equilibria in markets without ordered preferences and with a Riesz space of commodities. Journal of Mathematical Economics 13(2):165-169.

[126] Khan, M. A. and N. T. Peck. 1989. On the interiors of productions sets in infinite dimensional spaces. Journal of Mathematical Economics 18(1):29-39. 
[127] Khan, M. A. and R. Vohra. 1984. Equilibrium in abstract economies without ordered preferences and with a measure space of agents. Journal of Mathematical Economics 13(2):133-142.

[128] Khan, M. A. and R. Vohra. 1988. On approximate decentralization of Pareto optimal allocations in locally convex spaces. Journal of Approximation Theory 52:149-161.

[129] Khan, M. A. and N. C. Yannelis, eds. 1991. Equilibrium theory in infinte dimensional spaces. Number 1 in Studies in Economic Theory. Berlin: Springer-Verlag.

[130] Kim, T., K. Prikry, and N. C. Yannelis. 1989. Equilibria in abstract economies with a measure space of agents and with an infinite dimensional strategy space. Journal of Approximation Theory $56: 256-266$.

[131] Koopmans, T. C. 1957. Three essays on the state of economic science. New York: McGraw-Hill.

[132] Koopmans, T. C. 1960. Stationary ordinal utility and impatience. Econometrica 28:287-309.

[133] Kreps, D. M. 1981. Arbitrage and equilibrium in economies with infinitely many commodities. Journal of Mathematical Economics 8:15-35.

[134] Kuhn, H. W. 1956. A note on 'The law of supply and demand'. Mathematica Scandinavica 4:143-146.

[135] Kuhn, H. W. 1956. On a theorem of Wald. In H. W. Kuhn and A. W. Tucker, eds., Linear Inequalities and Related Systems, number 38 in Annals of Mathematics Studies, pages 265-274. Princeton University Press, Princeton.

[136] Levine, D. K. 1989. Infinite horizon equilibrium with incomplete markets. Journal of Mathematical Economics 18(4):357-376.

[137] Lucas, Jr., R. E. and E. C. Prescott. 1971. Investment under uncertainty. Econometrica 39:659681.

[138] Luenberger, D. G. 1994. Optimality and the theory of value. Journal of Economic Theory 63:147169.

[139] Magill, M. 1981. An equilibrium existence theorem. Journal of Mathematical Analysis and Applications 84:162-169.

[140] Magill, M. 1981. Infinite horizon programs. Econometrica 49(3):679-711.

[141] Majumdar, M. 1970. Some approximation theorems on efficiency prices for infinite programs. Journal of Economic Theory 2:399-410.

[142] Majumdar, M. 1972. Some general theorems on efficiency prices with an infinite dimensional commodity space. Journal of Economic Theory 5:1-12.

[143] Majumdar, M. 1974. Efficient programs in infinite dimensional spaces: A complete characterization. Journal of Economic Theory 7:355-368.

[144] Majumdar, M. and M. Kurz. 1972. Efficiency prices in an infinite dimensional space: A synthesis. Review of Economic Studies 39:47-158.

[145] Malinvaud, E. 1953. Capital accumulation and efficient allocation of resources. Econometrica $21: 233-268$.

[146] Malinvaud, E. T962. Efficient capital-accumulation: A Corrigendum. Econometrica 30:570-573.

[147] Mantel, R. 1974. On the characterization of aggregate excess demand. Journal of Economic Theory 7:348-353.

[148] Marimon, R. 1989. Stochastic turnpike property and stationary equilibrium. Journal of Economic Theory 47(2):282-306.

[149] Mas-Colell, A. 1974. An equilibrium existence theorem without complete or transitive preferences. Journal of Mathematical Economics 1:237-246. 
[150] Mas-Colell, A. 1975. A model of equilibrium with differentiated commodities. Journal of Mathematical Economics 2(2):263-295.

[151] Mas-Colell, A. 1985. The theory of general economic equilibrium: A differentiable approach. Cambridge: Cambridge University Press.

[152] Mas-Colell, A. 1986. The price equilibrium existence problem in topological vector lattices. Econometrica 54(5):1039-1053.

[153] Mas-Colell, A. 1986. Valuation equilibrium and Pareto optimum revisited. In [113], chapter 17, pages 317-331.

[154] Mas-Colell, A. and S. F. Richard. 1991. A new approach to the existence of equilibria in vector lattices. Journal of Economic Theory 53:1-11.

[155] Mas-Colell, A. and W. R. Zame. 1991. Equilibrium theory in infinite dimensional spaces. In [114].

[156] McCabe, P. J. 1981. On two market equilibrium theorems. Journal of Mathematical Economics 8:167-171.

[157] McFadden, D., A. Mas-Colell, R. Mantel, and M. K. Richter. 1974. A characterization of community excess demand functions. Journal of Economic Theory 9:361-374.

[158] McFadden, D., T. Mitra, and M. Majumdar. 1980. Pareto optimality and competitive equilibrium in infinite horizon economies. Journal of Mathematical Economics 7(1):1-26.

[159] McKenzie, L. W. 1954. On equilibrium in Graham's model of world trade and other competitive systems. Econometrica 22:147-161.

[160] McKenzie, L. W. 1955. Competitive equilibrium with dependent consumer preferences. In H. A. Antosiewicz, ed., Proceedings of the Second Symposium in Linear Programming, pages 277294, Washington, D.C. National Bureau of Standards and Directorate of Management Analysis, DCS/Comptroller, USAF.

[161] McKenzie, L. W. 1959. On the existence of general equilibrium for a competitive market. Econometrica 27:54-71.

[162] McKenzie, L. W. 1961. On the existence of general equilibrium: Some corrections. Econometrica 29:247-248.

[163] McKenzie, L. W. 1981. The classical theorem on existence of competitive equilibrium. Econometrica 29:819-841.

[164] Mehta, G. and E. Tarafdar. 1987. Infinite-dimensional Gale-Nikaidô-Debreu theorem and a fixedpoint theorem of Tarafdar. Journal of Economic Theory 41(2):333-339.

[165] Monteiro, P. K. 1987. Some results on the existence of of utility functions on path connected spaces. Journal of Mathematical Economics 16:147-156.

[166] Moore, J. 1975. The existence of 'compensated equilibrium' and the structure of the Pareto efficiency frontier. International Economic Review 16:167-300.

[167] Negishi, T. 1960. Welfare economics and existence of an equilibrium for a competitive economy. Metroeconomica 12:92-97.

[168] Neuefeind, W.: 1980. Notes on existence of equilibrium proofs and the boundary behavior of supply. Econometrica 48:1831-1837.

[169] Nikaidô, H. 1956. On the classical multilateral exchange problem. Metroeconomica 8:135-145.

[170] Nikaidô, H. 1957. A supplementary note to 'On the classical multilateral exchange problem'. Metroeconomica 9:209-210.

[171] Nikaidô, H. 1968. Convex structures and economic theory. Mathematics in Science and Engineering. New York: Academic Press. 
[172] Ostroy, J. M. 1984. On the existence of Walrasian equilibrium in large-square economies. Journal of Mathematical Economics 13(2):143-163.

[173] Peleg, B. 1970. Efficiency prices for optimal consumption plans (I). Journal of Mathematical Analysis and Applications 29:83-90.

[174] Peleg, B. 1970. Efficiency prices for optimal consumption plans (II). Israel Journal of Mathematics 9:222-234.

[175] Peleg, B. 1970. Efficiency prices for optimal consumption plans III. Journal of Mathematical Analysis and Applications 32:630-638.

[176] Peleg, B. and M. Yaari. 1970. Efficiency prices in infinite-dimensional space. Journal of Economic Theory 2:41-85.

[177] Peleg, B. and M. E. Yaari. 1970. Markets with countably many commodities. International Economic Review 11:369-377.

[178] Podczeck, K. forthcoming. Equilibria in vector lattices without ordered preferences and without uniform properness. Journal of Mathematical Economics.

[179] Prescott, E. C. and R. E. Lucas, Jr. 1972. A note on price systems in infinite dimensional space. International Economic Review 13:416-422.

[180] Prescott, E. C. and R. M. Townsend. 1984. General competitive analysis in an economy with private information. International Economic Review 25:1-20.

[181] Rader, T. 1963. The existence of a utility function to represent preferences. Review of Economic Studies 30(3):229-232.

[182] Radner, R. 1968. Competitive equilibrium under uncertainty. Econometrica 36:31-58.

[183] Raut, L. K. 1986. Myopic topologies on general commodity spaces. Journal of Economic Theory 39:358-367.

[184] Richard, S. F. 1989. A new approach to production equilibria in vector lattices. Journal of Mathematical Economics 18(1):41-56.

[185] Richard, S. F. and S. Srivastava. 1988. Equilibrium in economies with infinitely many consumers and infinitely many commodities. Journal of Mathematical Economics 17(1):9-21.

[186] Richard, S. F. and W. R. Zame. 1986. Proper preferences and quasi-concave utility functions. Journal of Mathematical Economics 15:231-247.

[187] Richter, M. K. 1980. Continuous and semi-continuous utility. International Economic Review 21:293-299.

[188] Rustichini, A. and N. C. Yannelis. 1991. Edgeworth's conjecture in economies with a continuum of agents and commodities. Journal of Mathematical Economics 20:307-26.

[189] Samuelson, P. A. 1958. An exact consumption-loan model of interest with or without the social contrivance of money. Journal of Political Economy 66:467-482.

[190] Scarf, H. E. 1967. The core of an N person game. Econometrica 35:50-69.

[191] Scarf, H. E. 1973. The computation of economic equilibria. New Haven, Connecticut: Yale University Press.

[192] Shafer, W. J. 1976. Equilibrium in economies without ordered preferences or free disposal. Journal of Mathematical Economics 3:135-137.

[193] Shafer, W. J. and H. F. Sonnenschein. 1975. Equilibrium in abstract economics without ordered preferences. Journal of Mathematical Economics 2:345-348.

[194] Shafer, W. J. and H. F. Sonnenschein. 1976. Equilibrium in economies with externalities, commodity taxation, and lump sum transfers. International Economic Review 17:601-611. 
[195] Shapley, L. and R. Vohra. 1991. On Kakutani's fixed point theorem, the K-K-M-S theorem and the core of a balanced game. Economic Theory 1:107-116.

[196] Shell, K. 1971. Notes on the economics of infinity. Journal of Political Economy 79:1002-1011.

[197] Simons, S. 1984. Minimaximin results with applications to ecooonomic equilibrium. Journal of Mathematical Economics 13:289-303.

[198] Sonnenschein, H. F. 1971. Demand theory without transitive preferences, with applications to the theory of competitive equilibrium. In J. S. Chipman, L. Hurwicz, M. K. Richter, and H. F. Sonnenschein, eds., Preferences, Utility, and Demand: A Minnesota Symposium, chapter 10, pages 215-223. Harcourt, Brace, Jovanovich, New York.

[199] Sonnenschein, H. F. 1972. Market excess demand functions. Econometrica 40:549-563.

[200] Sonnenschein, H. F. 1973. Do Walras' identity and continuity characterize the class of community excess demand functions? Journal of Economic Theory 6:345-354.

[201] Sonnenschein, H. F. 1977. Some recent results on the existence of equilibrium in finite purely competitive economies. In M. D. Intriligator, ed., Frontiers of quantitative economics, volume IIIA. North Holland, Amsterdam.

[202] Stigum, B. P. 1969. Competitive equilibrium under uncertainty. Quarterly Journal of Economics 83(4):533-561.

[203] Stigum, B. P. 1972. Competitive equilibria with infinitely many commodities. Metroeconomica 24:221-244.

[204] Stigum, B. P. 1973. Competitive equilibria with infinitely many commodities (II). Journal of Economic Theory 6:415-445.

[205] Stokey, N., R. E. Lucas, Jr., and E. C. Prescott. 1989. Recursive methods in economic dynamics. Cambridge, Mass.: Harvard University Press.

[206] Stroyan, K. D. 1983. Myopic utility functions on sequential economies. Journal of Mathematical Economics 11:267-276.

[207] Tarafdar, E. and G. Mehta. 1984. The existence of quasi-equilibria in a competitive economy. International Journal of Science and Engineering 1:1-12.

[208] Toussaint, S. 1984. On the existence of equilibria in economies with infinitely many commodities and without ordered preferences. Journal of Economic Theory 33:98-115.

[209] Uzawa, H. 1962. Walras' existence theorem and Brower's fixed point theorem. Economic Studies Quarterly 13.

[210] Van Zandt, T. 1990. Individual excess demands and equilibrium in exchange economies with infinitely many commodities. Princeton University. Manuscript.

[211] Vohra, R. 1987. On Scarf's theorem on the non-emptiness of the core: A direct proof through Kakutani's fixed point theorem. Working paper, Brown University.

[212] von Neumann, J. 1935-1936. Ueber ein okonomishches Gleichungssystem and und eine Verallgemeinerung des Brouwerschen Fixpunktsatzes. Ergebnisse eines Mathematischen Kolloquiums 8:73-83. Translation: "A Model of general economic equilibrium" Review of Economic Studies, 13(1945-1946).

[213] Wald, A. 1936. Uber die Produktionsgleichungen der okonomischen Wertlehre. Ergebnisse eines Mathematischen Kolloquiums 7:1-6. [Translation: "On the Production Equations of Economic Value Theory (Part 2)," in [37].].

[214] Walras, L. 1874. Elèments d'èconomie politique pure. Lausanne: Corbaz.

[215] Werner, J. 1987. Arbitrage and the existence of competitive equilibrium. Econometrica 55(6):14031418. 
[216] Wilson, C. A. 1981. Equilibrium in dynamic models with an infinity of agents. Journal of Economic Theory 24:95-111.

[217] Yannelis, N. C. 1985. On a market equilibrium theorem with an infinite number of commodities. Journal of Mathematical Analysis and Applications 108:595-599.

[218] Yannelis, N. C. 1991. The core of an economy without ordered preferences. In [129], pages 102-123.

[219] Yannelis, N. C. and N. D. Prabhakar. 1983. Existence of maximal elements and equilibria in linear topological spaces. Journal of Mathematical Economics 12(3):233-245.

[220] Yannelis, N. C. and W. R. Zame. 1986. Equilibria in Banach lattices without ordered preferences. Journal of Mathematical Economics 15(2):85-110.

[221] Zame, W. R. 1986. Markets with a continuum of traders and infinitely many commodities. Working paper, SUNY at Buffalo.

[222] Zame, W. R. 1987. Competitive equilibria in production economies with infinite-dimensional commodity space. Econometrica 55(5):1075-1108. 